\title{
Concurrent depletion of Vps37 proteins evokes ESCRT-I destabilization and profound cellular stress responses
}

\author{
Krzysztof Kolmus $^{1}$, Purevsuren Erdenebat ${ }^{1} \dagger$, Blair Stewig ${ }^{1} \dagger$, Ewelina Szymańska ${ }^{1}$, Krzysztof \\ Goryca $^{2} \uparrow \uparrow$, Edyta Derezińska-Wołek ${ }^{3,4}$, Anna Szumera-Ciećkiewicz ${ }^{3,4}$, Marta Brewińska- \\ Olchowik $^{5}$, Katarzyna Piwocka ${ }^{5}$, Monika Prochorec-Sobieszek ${ }^{3,4}$, Michał Mikula ${ }^{2}$, Marta \\ Miączyńska $^{1 *}$
}

\footnotetext{
Affiliations:

${ }^{1}$ Laboratory of Cell Biology, International Institute of Molecular and Cell Biology, 02-109, Warsaw, Poland

${ }^{2}$ Department of Genetics, Maria Skłodowska-Curie National Research Institute of Oncology, 02781, Warsaw, Poland

3 Department of Pathology and Laboratory Medicine, Maria Skłodowska-Curie National Research Institute of Oncology, 02-781, Warsaw, Poland

${ }^{4}$ Department of Diagnostic Hematology, Institute of Hematology and Transfusion Medicine, 02776, Warsaw, Poland

${ }^{5}$ Laboratory of Cytometry, Nencki Institute of Experimental Biology, 02-093, Warsaw, Poland

* Corresponding author: Tel: +48 2259707 25; Email: miaczynska@iimcb.gov.pl

$\uparrow$ These authors contributed equally.

$\dagger \dagger$ Present address: Next-Generation Sequencing Core Facility, Centre of New Technologies UW, 02-097, Warsaw, Poland
}

Running title: Vps37 depletion induces cell stress.

Key words: ESCRT, Vps37, Tsg101, colorectal cancer, inflammation, cell growth 


\section{SUMMARY STATEMENT}

Endosomal Sorting Complex Required for Transport (ESCRT)-I destabilization upon concurrent depletion of Vps37 proteins is linked to the activation of sterile inflammatory response and cell growth inhibition.

\section{ABSTRACT}

Molecular details of how endocytosis contributes to oncogenesis remain elusive. Our in silico analysis of colorectal cancer (CRC) patients revealed stage-dependent alterations in the expression of 113 endocytosis-related genes. Among them transcription of the Endosomal Sorting Complex Required for Transport (ESCRT)-I component VPS37B was decreased in the advanced stages of CRC. Expression of other ESCRT-I core subunits remained unchanged in the investigated dataset. We analyzed an independent cohort of CRC patients showing also reduced VPS37A mRNA and protein abundance. Transcriptomic profiling of CRC cells revealed nonredundant functions of Vps37 proteins. Knockdown of VPS37A and VPS37B triggered p21mediated inhibition of cell proliferation and sterile inflammatory response driven by the Nuclear Factor (NF)- $\kappa \mathrm{B}$ transcription factor and associated with mitogen-activated protein kinase signaling. Co-silencing of VPS37C further potentiated activation of these independently induced processes. The type and magnitude of transcriptional alterations correlated with the differential ESCRT-I stability upon individual and concurrent Vps37 depletion. Our study provides novel insights into cancer cell biology by describing cellular stress responses that are associated with ESCRT-I destabilization, which might occur in CRC patients. 


\section{INTRODUCTION}

Genetic alterations induce the reprogramming of intracellular signaling, which is a driving force of tumorigenesis. The duration of signal transduction is dependent on endocytosis (Floyd and De Camilli, 1998; Mosesson et al., 2008; Schmid, 2017). Some mechanisms for tumor cell-specific changes in the activity of endocytic machinery components that affect intracellular signaling have been already identified (Barbieri et al., 2016; Di Fiore and von Zastrow, 2014; Mellman and Yarden, 2013). For instance, many tumors exhibit deregulated expression of the ubiquitination machinery and small GTPases that control the rate of receptor degradation and recycling, respectively (Porther and Barbieri, 2015). However, despite the abundance of publicly available data, such as those deposited in The Cancer Genome Atlas [TCGA] (Weinstein et al., 2013), little has been done to systematically analyze the expression of receptor trafficking regulators in tumors and across tumor stages. This knowledge could potentially facilitate patients' stratification for treatment with bioengineered macromolecules delivered through receptor-mediated endocytosis (Tashima, 2018).

An important group of trafficking regulators constitute four sequentially acting Endosomal Sorting Complexes Required for Transport (ESCRT-0, ESCRT-I, ESCRT-II, and ESCRT-III) and accessory proteins, among others Vps4A and Vps4B. The ESCRT machinery mediates receptor degradation not only by recognition and local clustering of ubiquitinated cargo on endosomes but also through membrane deformation and scission to form intraluminal vesicles (ILVs). Many rounds of ILV formation create multivesicular bodies that fuse with lysosomes leading to cargo degradation. In addition, ESCRTs contribute to cytokinesis, autophagy, virus budding, exovesicle release, and repair of plasma and intracellular membranes (Hurley, 2015; Olmos and Carlton, 2016; Szymanska et al., 2018; Vietri et al., 2020). Despite the wellestablished roles of ESCRT components in maintaining cell homeostasis, much less is known about their contribution to tumorigenesis (Alfred and Vaccari, 2016; Gingras et al., 2017; Mattissek and Teis, 2014) and the underlying molecular mechanism has been clarified only in a couple of cases (Manteghi et al., 2016; Sadler et al., 2018). For instance, we demonstrated that the expression of $V P S 4 B$, encoding ESCRT-associated ATPase, is decreased in colorectal cancer (CRC) and VPS4B-deficient cells are critically dependent on the Vps4A protein. This synthetic lethality between VPS4 paralogs triggers stress-associated sterile inflammatory response and 
immunogenic cell death and thus may be used as a basis for personalized therapy (Szymanska et al., 2020).

ESCRT-I is a heterotetramer composed of three core components (Tsg101, Vps28 and one of four Vps37 family members) and a single auxiliary protein (UBAP-1, Mvb12A or Mvb12B) (Stefani et al., 2011; Wunderley et al., 2014). At least under some conditions two of its subunits (Tsg101 and Vps37A) have been identified as putative tumor suppressors (Li and Cohen, 1996; Moberg et al., 2005; Xu et al., 2003). In parallel, high-throughput screens for cancer vulnerability within the DepMap project (Behan et al., 2019) demonstrated that multiple cancer cell lines display a reduced fitness upon TSG101 knockout, whilst the effect of perturbed expression of VPS28, VPS37A, VPS37C or UBAP1 genes is cell type-dependent.

Tsg101 and Vps37A are not only regulators of vesicular trafficking but also other biological processes, such as transcription and autophagy (Bache et al., 2004; Bishop et al., 2002). Transcriptomic analysis of Tsg101-depleted cancer cells revealed increased expression of the prototypical Nuclear Factor-кB (NF-кB)-dependent genes without exogenous stimulation (Brankatschk et al., 2012). We dissected the molecular basis of this phenomenon showing that the absence of Tsg101 or Vps28 led to the accumulation of ligand-free cytokine receptors on endosomes because of disturbed sorting into ILVs and degradation of cargo. The proximity of accumulated receptors on endosomes evoked their oligomerization to trigger NF-KB signaling (Banach-Orlowska et al., 2018; Maminska et al., 2016). However, none of the Vps37 proteins incorporated into ESCRT-I was identified as a genuine regulator of the NF-kB pathway.

NF-KB is a family of ubiquitously expressed transcription factors, whose activation is a hallmark of inflammation often associated with cancer (Taniguchi and Karin, 2018). These transcription factors mediate also other biological processes, such as proliferation (Zhang et al., 2017). There are two interconnected NF-KB signaling cascades. The canonical NF-KB pathway culminates in the phosphorylation and degradation of the I $\mathrm{B} \alpha$ inhibitor, that allows an active p65-p50 NF-kB dimer to translocate into the nucleus. The non-canonical signaling cascade marks the p100 NF$\mathrm{KB}$ precursor for proteasomal processing to $\mathrm{p} 52$ to form transcriptionally active complexes with RelB (Hayden and Ghosh, 2008). Although endocytic trafficking and NF-kB inflammatory signaling are important in carcinogenesis, the molecular links between them are poorly studied. 
81 Here, we systematically analyzed the expression of endocytosis regulators across stages of CRC using publically available data and found decreased expression of VPS37 paralogs. As no genome-wide expression studies have explored the cellular consequences of individual and concurrent depletion of Vps37 family members, we investigated their roles focusing on the processes related to cell growth and inflammatory response. Our findings reveal the importance of VPS37 paralogs in orchestrating cell homeostasis through maintaining the stability of ESCRTI.

\section{RESULTS}

\section{Expression of $V P S 37 B$ is decreased in advanced colorectal cancer}

CRC is a leading cause of cancer-associated deaths worldwide as it is often diagnosed in advanced stages when patients display clinical symptoms (Siegel et al., 2018). Aberrant endosomal trafficking in CRC has been linked to adverse phenotype and resistance to therapies (Gargalionis et al., 2015). In order to gain insight into transcriptional changes of genes involved in endosomal trafficking in CRC, we mined the TCGA data against a custom-made list (Table S1) of components whose biological function was related to endocytic transport. Matched normal and cancer transcriptomic samples of human CRC cohorts (colon adenocarcinoma [COAD] and rectal adenocarcinoma [READ]) with available clinicopathological information (31 patients in total) were divided based on tumor staging to early (Stage I and II; 19 patients) or advanced (Stage III and IV; 12 patients) disease stage pools (Fig. 1A). Out of the 445 endocytic genes tested, 410 genes fulfilled normalization criteria of the present analysis (see Materials and Methods). We observed differential gene expression of 113 genes at different stages of CRC when compared to levels transcribed in matched healthy colon tissue (Fig. 1B). Differential expression of 20 genes was unique for the early stages (Table S2). 21 genes were differentially expressed in the advanced stages of tumorigenesis (Table S3). In addition to decreased mRNA abundance of VPS4B, which we studied before (Szymanska et al., 2020), we detected reduced expression of an ESCRT-I component - VPS37B - in the advanced stages of CRC (Fig. 1C).

Since we and others had demonstrated that two out of three core ESCRT-I components, namely Tsg101 and Vps28, restrict NF-кB-dependent transcription (Brankatschk et al., 2012; Maminska 
110 et al., 2016), we analyzed expression of genes encoding the core ESCRT-I subunits in normal 111 colorectal tissue samples from the TCGA datasets. Using the transcripts per million (TPM) 112 metrics to normalize expression data with respect to gene length and sequencing depth, we 113 observed that colorectal tissue and its cancer counterpart expressed high levels of TSG101, $114 V P S 28, V P S 37 B$, followed by comparable levels of $V P S 37 A$ and $V P S 37 C$, and negligible levels 115 of VPS37D. Differential expression analysis of CRC samples compared against matching healthy 116 tissue controls revealed that transcription of $V P S 37 B$ tended to be decreased in the early stages of $117 \mathrm{CRC}$, and was reduced 1.61-fold in the advanced stages (Table S4). Expression of VPS28 was 118 slightly decreased in the early stages of tumorigenesis (Table S4). On the other hand, levels of 119 TSG101, VPS37A and VPS37C were stably expressed across tumor stages (Fig. 1D, Table S4).

120 In summary, expression of $V P S 37 B$ is decreased during progression from early to advanced 121 stages of CRC, whilst transcription of the remaining ESCRT-I components is unchanged.

mRNA and protein abundance of $V P S 37 A$ and $V P S 37 B$ paralogs is decreased in CRC patient cohorts

Since the expression of $V P S 37 B$ was decreased in samples deposited in the TCGA database and VPS37A mRNA and protein abundance was previously shown to be reduced in CRC patients (Chen et al., 2018; Miller et al., 2018; Vasaikar et al., 2019), we performed qRT-PCR analysis of VPS37 mRNA levels using an independent set of CRC samples from our previous study (Mikula et al., 2011; Skrzypczak et al., 2010). We observed a significant decrease in VPS37B and VPS37A mRNA abundance in adenocarcinoma (Fig. 2A).

To assess whether transcriptional alterations at mRNA level correlate with the diminished abundance of $\operatorname{Vps} 37$ proteins in CRC, we performed immunohistochemistry (IHC) staining of Vps37A and Vps37B in an array setup consisting of 100 pairs of treatment-naïve primary CRC samples and non-cancerous colon tissue using specific antibodies (Fig. S1A-E). We evaluated the tissue arrays using a semi-quantitative scoring method based on staining intensity.

Both Vps37A and Vps37B displayed strong cytoplasmic staining in normal colon tissue (Fig. 2B). Out of the 100 investigated patient samples, protein staining of Vps37A was decreased to the medium intensity level $(3+\rightarrow 2+)$ in cancerous tissue of all examined patients. Vps37B 
139 protein staining was decreased to the medium intensity level $(3+\rightarrow 2+)$ in $70 \%$ of patients and to 140 weak intensity levels $(3+\rightarrow 1+)$ in $30 \%$ of patients (Fig. 2C). Since the analyzed group of 141 treatment-naïve CRC patients was very homogenous with respect to pathological tumor (pT) 142 status, pathological nodes $(\mathrm{pN})$ and disease grade (Table S5), we could not correlate Vps37 staining intensity with clinical disease staging.

144 Overall, these results corroborated the finding of our bioinformatics analysis that the abundance 145 of $V P S 37 B$ is decreased at transcriptional and protein levels in CRC. They further mounted evidence for reduced mRNA and protein levels of VPS37A in CRC.

\section{Concurrent depletion of Vps37 proteins induces multifaceted transcriptional responses in}

\section{CRC cells}

150

151

152

153

154

155

156

157

158

159

160

161

162

163

164

165

166

167

Humans have four VPS37 genes whose protein products display distinct domain architecture suggesting partly different functions in the cells. They all possess the Mod(r) domain mediating interaction with the remaining core ESCRT-I subunits. Whereas only Vps37A has the ubiquitinbinding UEV domain, the other members contain the proline-rich region (PRR) essential for protein-protein interactions (Fig. 3A). To study the cellular functions of Vps37 paralogs, we used an in vitro model of human colon cancer DLD1 cell line. The expression levels of VPS37 paralogs in the DLD1 cell line reflect those observed in samples of CRC patients deposited in TCGA (Dou et al., 2016), as well in our data (GSE152195).

To gain insights into the molecular consequences of individual and concurrent depletion of Vps37 proteins on cellular homeostasis, we performed RNA-Seq in DLD1 cells. We first verified high knockdown efficiency and selectivity of siRNA (two independent sequences per target) by measuring the protein abundance of the three paralogs in DLD1 cells (Fig. S2A-C). Despite the sequence similarity between $V P S 37 A, V P S 37 B$ and $V P S 37 C$ (Fig. 3A), we could selectively silence the expression of each individual paralog or their combinations. As we observed certain differences in the silencing efficiency, we used single strongly acting siRNAs for RNA-Seq to knockdown VPS37 paralogs individually or in double and triple combinations (7 conditions), compared to non-transfected cells (NT) and cells transfected with a combination of non-targeting siRNA (siCTRL\#1). Nevertheless, all subsequent experiments were performed 
with two independent siRNA sequences per target. We considered genes to be differentially expressed when their expression was either below 0.667-fold or above 1.5-fold, and adjusted $P<0.05$ when normalizing against the two control conditions - siCTRL\#1 and non-transfected cell (NT) (Fig. S2D).

We observed that co-silencing of VPS37A, VPS37B and VPS37C (abbreviated as VPS37ABC) elicited the greatest transcriptional changes (1277 genes). Pronounced changes (781 genes) were also detected after concurrent silencing of $V P S 37 A B$ indicating the importance of these two subunits in maintaining homeostasis of DLD1 cells. Conversely, a limited number of genes underwent transcriptional changes upon other silencing combinations of VPS37 family members (Fig. S2D). Hierarchical clustering of all investigated conditions on a set of differentially expressed genes after individual, double and triple silencing demonstrated that the branch containing siVPS37ABC\#1 and siVPS37AB\#1 was clearly distinct from the remaining conditions (Fig. S2E). We further observed that the pools of genes induced upon single depletion of Vps37A, Vps37B and Vps37C were largely non-overlapping (Fig. S2F), indicating that multifaceted transcriptional responses induced upon co-depletion of Vps37 proteins stem from the accumulation of paralog-specific defects in the cells.

Differentially expressed genes under each silencing conditions were subjected to the Gene Ontology (GO) analysis of biological processes. We identified biological processes only in transfection conditions with knockdown of the VPS37A paralog: siVPS37A\#1, siVPS37AB\#1, VPS37AC\#1 and VPS37ABC\#1. Among the top 15 gene signatures (whose order was determined based on a number of genes in the cluster upon $V P S 37 A B C$ silencing) were processes related to cell migration, cellular signaling, inflammatory response, cell growth, and adhesion (Fig. 3B). We further focused on the "inflammatory response" (GO:0006954) and "regulation of growth" (GO:0040008) gene clusters. The inflammatory response heatmap contained genes encoding cytokines $(C X C L 8)$, adhesion molecules (ICAM1), and negative regulators of NF- $\mathrm{BB}$ signaling (TNFAIP3). In-depth interrogation of genes linked to the "regulation of growth" showed the presence of cyclin-dependent kinase inhibitors (CDKN1A,CDKN2D) and regulators of cell growth (HMGA2, SFN) (Fig. 3C,D). To determine the signaling pathways associated with inflammatory response and regulation of cell growth, we conducted a pathway network analysis using the Reactome Database. Our analysis of differentially expressed genes yielded enrichment 
198 of annotations related to signaling initiated by cytokines, receptor tyrosine kinases, and G-protein

199

200

201

202

203

204

205

206

207

208

209

210

211

212

213

214

215

216

217

218

219

220

221

222

223

224

225

226

coupled response and involving mitogen-activated protein kinases (MAPK) and PI3K/Akt (Fig. $3 \mathrm{E})$.

Collectively, these data point to largely non-redundant cellular functions of VPS37 paralogs. The type and magnitude of transcriptional responses after their co-silencing are the cumulative response to perturbations of individual functions executed by Vps37 proteins. Concurrent knockdown of $V P S 37 A B$ profoundly affects gene expression patterns linked to "inflammatory response" and "regulation of cell growth" and additional silencing of VPS37C paralog on top of $V P S 37 A B$ knockdown further potentiates perturbations in gene transcription. These data suggested that Vps37 depletion activates multifaceted stress responses in the cells.

\section{Inflammatory gene expression is induced upon concurrent depletion of Vps37 proteins}

To validate our RNA-Seq analysis, we selected the most pronouncedly induced genes (based on the fold change values) from to the inflammatory response cluster that represented different classes of molecules (cyto-/chemokine, adhesion molecule, classical and non-classical regulators of NF- $\kappa$ B signaling; Fig. 3C, GSE152195), and performed a qRT-PCR analysis of DLD1 cells subjected to individual or concurrent silencing of VPS37 paralogs. We found that knockdown of VPS37A induced transcription of TNFAIP3 (encoding A20) as well as ICAM1 and had modest, yet insignificant, the effect on the expression of CXCL8 (encoding IL-8) and NFKBIA (encoding $\mathrm{I} \kappa \mathrm{B} \alpha$ ) (Fig. S3A-D). Silencing of either $V P S 37 B$ or $V P S 37 C$ did not affect any of the investigated genes. Concurrent Vps37AB depletion significantly promoted $C X C L 8, N F K B I A$ and TNFAIP3 transcription and modestly affected ICAM1 transcription. Expression of the TNFAIP3 gene was also induced by concurrent $V P S 37 A C$ silencing. Finally, we observed that concurrent knockdown of VPS37ABC increased the expression of CXCL8, ICAM1, NFKBIA, and TNFAIP3 comparable to concurrent Vps37AB depletion (Fig. S3A-D).

Since the magnitude of transcriptional changes in DLD1 cells was modest, we tested the expression of the same genes in a poorly differentiated RKO carcinoma cell line. We found that knockdown of VPS37A had a modest, yet insignificant, effect on the expression of $C X C L 8$ and ICAM1, whereas it did not affect TNFAIP3 or NFKBIA transcription (Fig. 4A-D). Silencing of 
either $V P S 37 B$ or $V P S 37 C$ did not affect the expression of any of the investigated genes. Concurrent VPS37AB silencing induced an increase of CXCL8, ICAM1, TNFAIP3, and NFKBIA expression. Neither knockdown of VPS37AC nor VPS37BC substantially affected the transcription of the investigated targets (Fig. 4A-D). We observed that concurrent depletion of Vps37ABC had further positive effects on the magnitude of CXCL8, ICAM1, NFKBIA and TNFAIP3 transcription compared to the Vps37AB-depleted cells (Figs 4A-D, S3E-G). Since the effects of individual and concurrent depletion of $\mathrm{Vps} 37$ proteins on gene expression were more pronounced in RKO cells, we decided to use it as the main model system in the subsequent experiments.

In summary, we found that concurrent depletion of Vps37 proteins, in particular Vps37AB and Vps37ABC, activates transcription of multiple classes of inflammatory genes, while knockdown of individual VPS37 paralogs induces only minor changes in their expression. We also noted that the rate of gene transcription is cell- type dependent.

\section{Concurrent knockdown of VPS37 paralogs induces NF-кB signaling and MAPK cascade}

We and others previously revealed that depletion of the core ESCRT-I subunits - Tsg101 and Vps28 - induced NF-кB-driven inflammatory response (Brankatschk et al., 2012; Maminska et al., 2016). As our RNA-Seq analysis yielded the "inflammatory response" gene cluster upon siVPS37AB\#1 and siVPS37ABC\#1 (Fig. 3C), we combined genes from each cluster into a single list and subjected them for transcriptional motif-enrichment analysis using RcisTarget (Aibar et al., 2017). For each gene, the promoter region of $500 \mathrm{bp}$ upstream and $100 \mathrm{bp}$ downstream to the transcription starting site was investigated with the transcription factor binding sites (TFBS) matrices available in the JASPAR database. Our in silico analysis revealed enrichment of TFBS for the members of NF- $\mathrm{BB}$, FOS, and AP1 transcription factors. The consensus sequence for RELA/p65 with the highest normalized enrichment score was the top annotated TFBS (Table 2).

Because concurrent silencing of VPS37 paralogs enhanced the expression of NF- $\kappa \mathrm{B}$ target genes (Figs 4A-D, S3A-D, Table 2), we explored the molecular basis of these effects. To this end we measured p65 phosphorylation and p100 to p52 processing as hallmarks of canonical and non- 
256

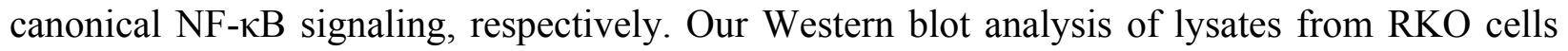
with silencing of individual VPS37 paralogs as well as VPS37AC and VPS37BC showed no significant induction of any branch of the NF- $\mathrm{B}$ pathway (Fig. 4E-G). VPS37AB knockdown induced p65 phosphorylation and cleavage of p100 to p52. Co-silencing of $V P S 37 A B C$ induced p65 phosphorylation, cleavage of p100 to p52 (Fig. 4E, G), and compared to Vps37AB depletion also increased p100 levels (Fig. 4F).

MAPKs cooperate with NF- $\kappa \mathrm{B}$ in driving inflammation (Hoesel and Schmid, 2013). Since signaling enrichment analysis of our transcriptomics data pointed to increased expression of genes whose products regulate the MAPK cascade, we tested phosphorylation of JNK, p38 and ERK as hallmarks of MAPK activation. Using Western blotting, we found that silencing of individual VPS37 paralogs did not affect JNK, p38, and ERK phosphorylation (Fig. 4H-J). Knockdown of $V P S 37 A B$ in RKO cells had minor positive, yet insignificant, effect on phosphorylation of JNK and p38 MAPK but did not activate ERK. Neither VPS37AC nor $V P S 37 B C$ silencing activated JNK, p38 and ERK (Fig. 4H-J). Concurrent Vps37ABC depletion induced JNK and p38 phosphorylation but again it did not activate ERK (Fig. 4H-J).

Overall, we concluded that depletion of $\mathrm{Vps} 37 \mathrm{ABC}$ is associated with the activation of canonical and non-canonical NF- $\mathrm{BB}$ signaling as well as JNK and p38 MAPK.

\section{Cell proliferation and colony forming ability of CRC cells are inhibited after concurrent knockdown of VPS37 paralogs}

As GO analysis of biological processes revealed the regulation of growth gene cluster (Fig. 3D, GSE152195), we examined the effect of differential depletion of Vps37 proteins on cell growth using a short-term BrdU proliferation assay and a long-term colony formation assay in DLD1 and RKO cells.

Using the proliferation assay, we observed that single silencing of $V P S 37 A$ modestly decreased the proliferation rate, which was statistically significant only in RKO cells (Fig. 5A,B). Knockdown of neither $V P S 37 B$ nor $V P S 37 C$ altered growth of DLD1 and RKO cells. Silencing of $V P S 37 A B$ significantly inhibited DLD1 and RKO cell proliferation, whilst knockdown of $V P S 37 A C$ and $V P S 37 B C$ had no impact. The strongest inhibition of cell proliferation was seen 
upon $V P S 37 A B C$ knockdown (Fig. 5A,B). In line with the results of proliferation assay, depletion of Vps37A alone or co-silencing of $V P S 37 A B$, VPS37AC and $V P S 37 A B C$ inhibited ability of DLD1 and RKO cells to form colonies in the clonogenic assay performed 14 days after siRNA transfection (Figs 5C-D, S4A-B). In parallel, we checked the impact of TSG101 silencing on cell proliferation and colony formation. Its knockdown in RKO cells inhibited both processes, comparably to concurrent silencing of VPS37 paralogs (Fig. S4C-E).

In conclusion, we found that concurrent depletion of Vps37 proteins has detrimental effects on cancer cell growth in vitro and the phenotype of Tsg101-depleted cells closely resembles the one observed in $V P S 37 A B C$ knockdown cells. Our results also indicate that growth rate is primarily dependent on the expression of both VPS37A and VPS37B. Additional co-silencing of VPS37C potentiated proliferation and colony-forming defects of $V P S 37 A B$ knockdown. In contrast, longterm growth rate appears to be largely dependent on Vps37A.

\section{Concurrent depletion of Vps37 proteins induces p21-mediated cell cycle arrest}

Accelerated division of tumor cells is among others a result of abnormal activity of cyclins and cyclin-dependent kinase inhibitors (CDKNs) (Bonelli et al., 2014). As our RNA-Seq data revealed increased expression of three genes encoding CDKNS (CDKN1A,CDKN2B, and $C D K N 2 D$, GSE152195, Fig. 3D), we used qRT-PCR to corroborate their changed transcription in RKO and DLD1 cells subjected to individual and concurrent silencing of VPS37 paralogs. In RKO cells, knockdown of $V P S 37 A$ did not affect $C D K N 1 A$ or $C D K N 2 B$ transcription (Fig. 5E-F) but modestly induced the expression of CDKN2D (Fig. 5G). Silencing of either VPS37B or $V P S 37 C$ did not change transcription of any of the analyzed genes. We found that $V P S 37 A B$ silencing increased $C D K N 1 A, C D K N 2 B$, and $C D K N 2 D$ transcription; yet in the case of $C D K N 2 B$, the increase was observed for only one pair of siRNA and did not reach statistical significance. Knockdown of neither VPS37AC nor VPS37BC paralogs had an impact on the investigated target genes (Fig. 5E-G). Instead, concurrent depletion of Vps37ABC further increased transcription of all investigated $C D K N$ s compared to concurrent depletion of $\mathrm{Vps} 37 \mathrm{AB}$ (Fig. 5E). In line, we found a similar pattern of $C D K N$ expression in differentially transfected DLD1 cells; however, we could not corroborate enhanced CDKN2D transcription upon co- 
314 depletion of Vps37 proteins that we initially identified in our RNA-Seq analysis (Fig. S4F-H).

315 Finally, we observed that the transcription pattern of CDKNs after depletion of Tsg101 in RKO

316 cells paralleled those observed for $C D K N 1 A$ and $C D K N 2 B$ expression after concurrent

$317 V P S 37 A B C$ silencing. In this case, silencing of TSG101 did not induce CDKN2D expression

318 (Fig. S4I).

319 Increased expression of $C D K N s$ after concurrent Vps37ABC depletion suggests an impact on the

320 proliferation rate and cell cycle progression. CDKN1A encodes p21, which inhibits cell cycle

321 progression in the G1, S and G2 phases, whilst $C D K N 2 B$ and $C D K N 2 D$ encode p15 ${ }^{\mathrm{INK} 4 \mathrm{~B}}$ and

$322 \mathrm{p} 19^{\mathrm{INK} 4 \mathrm{D}}$, respectively, which inhibit complexes formed by cyclin D and halt cell cycle in the G1

323 phase (Bonelli et al., 2014). Thus, we evaluated the proliferation of Vps37ABC-depleted RKO

324 cells upon co-silencing of the CDKN1A gene, which was the most pronouncedly induced in our

325 qRT-PCR analysis. We observed that concurrent knockdown of $V P S 37 A B C$ and $C D K N 1 A$ partly

326 rescued cell proliferation, corroborating the inhibitory impact of $\mathrm{p} 15^{\mathrm{INK} 4 \mathrm{~B}}$ and $\mathrm{p} 19^{\mathrm{INK} 4 \mathrm{D}}$ on cell

327 division (Fig. 5H). In line, p21 depletion in cells transfected with siRNA against TSG101

328 improved RKO cell proliferation (Fig. S4J).

329 To gain further insights into the inhibition of cell growth after differential silencing of VPS37

330 paralogs, siRNA-transfected RKO cells were stained with propidium iodide and cell cycle was

331 analyzed by flow cytometry. We observed that knockdown of either $V P S 37 B$ or $V P S 37 C$ did not

332 change cell cycle progression as indicated by the unaltered percentage of cells in the G0/G1 and

333 S phases (Fig. 5I). Knockdown of VPS37A increased the percentage of cells in the G0/G1 phase

334 and decreased the number of cells in the S phase (Fig. 5I). The impact of Vps37AC-depletion

335 closely paralleled that observed after VPS37A silencing. In contrast to Vps37BC-depleted cells,

336 whose cell cycle progression was not affected, concurrent knockdown of VPS37AB resulted in

337 the increased number of cells in the G0/G1 phase and a drop in the S phase (Fig. 5I). The

338 proportion of cells in the G0/G1 phase after combined silencing of $V P S 37 A B C$ was comparable

339 to that observed in Vps37AB-depleted cells (Fig. 5I). Finally, silencing of TSG101 closely

340 paralleled the effects observed after $V P S 37 A B C$ knockdown (Fig. S4K). None of the analyzed

341 silencing conditions (involving VPS37 paralogs and TSG101) altered the percentage of cells in

342 the G2/M phase (Figs 5I, S4K). 
In summary, our data uncovered that concurrent depletion of Vps37 proteins induces the expression of three $C D K N s$, which cooperatively halt cell cycle in the G1 phase. Moreover, the phenotype of Tsg101-depleted cells closely resembles the one observed in VPS37ABC knockdown cells. We further demonstrated that $C D K N$ expression and cell cycle progression are primarily dependent on Vps37A and modulated by the presence of other family members.

\section{NF-кB response and p21-mediated growth arrest are induced independently after depletion of VPS37 paralogs}

We next investigated the molecular basis for the induction of NF- $\mathrm{\kappa B}$ response and $\mathrm{p} 21$-mediated growth arrest after depletion of all three Vps37 proteins in RKO cells. Since CDKN1A encoding p21 was the most potently affected gene in our qRT-PCR analysis (Fig. 5E) and its knockdown in Vps37ABC-depleted RKO cells partly rescued their proliferation (Fig. 5H), we used it as readout to assess the relationship between inflammatory response and cell growth arrest in Vps37ABC-depleted cells.

We first investigated the time course of changes in p21 levels and activation of the NF- $\mathrm{kB}$ pathway components after concurrent $V P S 37 A B C$ silencing in RKO cells. We observed that depletion of Vps37ABC increased p21 abundance after $24 \mathrm{~h}$ and $72 \mathrm{~h}$ post-transfection (Fig. 6A). We found rapid phosphorylation of p65 in Vps37ABC-depleted cells, $24 \mathrm{~h}$ and $72 \mathrm{~h}$ posttransfection; however, after $24 \mathrm{~h}$ p 65 phosphorylation did not reach statistical significance (Fig. 6B). The abundance of $\mathrm{p} 100 / \mathrm{p} 52$ increased from $24 \mathrm{~h}$ to $72 \mathrm{~h}$ post-transfection in cells with $V P S 37 A B C$ knockdown (Fig. 6C,D). Throughout 24-72 h post-transfection, abundance of Vps37A, Vps37B and Vps37C gradually decreased after $24 \mathrm{~h}$ and remained undetectable after 72 $\mathrm{h}$ post-transfection (Fig. S5A-C). These data showed that the activation of NF- $\mathrm{kB}$ signaling and production of $\mathrm{p} 21$ occurred within the similar timeframe after Vps37ABC depletion, thus none of these processes preceded each other.

In certain cell types, the canonical NF-kB pathway is crucial for $C D K N 1 A$ transcription (Ledoux and Perkins, 2014) and we verified whether increased CDKN1A expression after co-depletion of Vps37 proteins required the canonical NF- $\mathrm{kB}$ subunit p65 (encoded by RELA). Knockdown of RELA in Vps37ABC-depleted cells did not affect CDKN1A expression (Fig. 6E), although it 
372 almost completely blunted transcription of CXCL8 (Fig. S5D) and substantially reduced expression of ICAM1, two prototypical NF- $\kappa \mathrm{B}$ target genes (Figs S5E, S5F-I). These results suggest that negative effects on cell growth stemming from co-silencing of VPS37 paralogs are not consequences of induction of canonical NF- $\kappa$ B signaling.

p21 modules NF-kB signaling in immune cells (Rackov et al., 2016; Trakala et al., 2009) but whether similar mechanisms occur in CRC cells has not been assessed. Thus, we explored whether silencing of $C D K N 1 A$ affected phosphorylation of p65 and processing of p100 to p52 upon Vps37ABC depletion. As assessed by Western blot, co-silencing of VPS37 paralogs and $C D K N 1 A$ did not affect the levels of p65 phosphorylation and p100 to p52 processing compared to co-silencing of VPS37 paralogs alone (Figs 6F-H, S5J-M). We also checked whether p21 depletion modulated the rate of inflammatory gene expression in Vps37ABC-depleted cells. Cosilencing of CDKN1A inhibited transcription of only CXCL8 but not ICAM1, TNFAIP3 and NFKBIA (Fig. 6I-L). These results showed no modulatory impact of $\mathrm{p} 21$ on NF- $\mathrm{KB}$ signaling and three out of four investigated target genes.

Overall, we concluded that in CRC cells with co-depletion of Vps37 proteins the induction of $\mathrm{NF}-\kappa \mathrm{B}$ inflammatory response and $\mathrm{p} 21$-mediated cell growth inhibition are two independent processes. These data point out that cell growth arrest is not caused by activation of inflammatory response.

\section{ESCRT-I is destabilized after either concurrent depletion of Vps37 proteins or TSG101 silencing}

We speculated that the type and magnitude of transcriptional responses after individual and concurrent silencing of VPS37 paralogs might be attributed to distinct ESCRT-I stability. It was previously shown that knockdown of some ESCRT-I core components induced partial or complete degradation of other complex subunits (Stefani et al., 2011; Wunderley et al., 2014); yet, a detailed characterization of all ESCRT-I subunits after individual and concurrent Vps37 proteins depletion has not been performed so far.

First, we checked whether knockdown of individual ESCRT-I components affected the stability of its remaining subunits expressed in CRC cells. Western blot analysis of lysates from RKO 
401 cells revealed that depletion of either Tsg101 or Vps28 destabilized each other (Fig. S6A,B) as

402

403

404

405

406

407

408

409

410

411

412

413

414

415

416

417

418 well as Vps37A, Vps37B, Vps37C, Mvb12A, Mvb12B, and lowered UBAP-1 protein abundance (Fig. S6C-H). We observed that silencing of VPS37A diminished UBAP-1 protein abundance indicating that ESCRT-I complexes containing Vps37A preferentially incorporate UBAP-1 (Fig. S6F). Depletion of Vps37B reduced the abundance of Tsg101 protein (Fig. S6A) and partially Mvb12A (Fig. S6G). Conversely, silencing of MVB12A decreased Vps37B (Fig. S6D), indicating partnering preference between these subunits. We did not observe any relationship between the stability of Vps37C and Mvb12 proteins (Figs S6E, S6G-H).

We next analyzed the stability of ESCRT-I core and auxiliary subunits upon concurrent silencing of two or three VPS37 paralogs. Co-depletion of Vps37AB or Vps37BC proteins decreased Tsg101 and Vps28 abundance, whilst the effects of VPS37AC knockdown were less potent (Fig. 7D,E). Knockdown of all three VPS37 genes led to the complete destabilization of Tsg101 and Vps28 proteins (Fig. 7D-E). Protein abundance of UBAP-1 was decreased in all silencing combinations involving VPS37A (Fig. 7F) corroborating the results of individual VPS37A knockdown (Fig. S6F). Similarly, Mvb12A abundance was reduced whenever cells were depleted of Vps37B (Fig. 7G), whilst such effect was less pronounced for Mvb12B (Fig. 7H). Silencing of all VPS37 genes depleted both Mvb12 proteins (Fig. 7G,H). Notably, the stability of the core and auxiliary ESCRT-I subunits after concurrent $V P S 37 A B C$ knockdown closely resembled the effects of TSG101 silencing (Fig. S6A).

In summary, these data show an inter-dependability of ESCRT-I subunits for maintaining the complex stability. They indicate that the incorporation of auxiliary subunits is selective with respect to their Vps37 partners (Vps37A with UBAP-1 and Vps37B with Mvb12A). We also found that simultaneous interference with VPS37A and VPS37B expression induces pronounced decrease in ESCRT-I stability and Vps37C depletion only slightly magnifies this effect. Our results further argue that the type and magnitude of transcriptional responses after differential depletion of Vps37 proteins correlate with the abundance of core and accessory ESCRT-I components.

\section{DISCUSSION}


430 Tumors develop various mechanisms to prolong exposure of plasma membrane receptors to ensure constitutive signaling that is beneficial for their growth. One of such mechanisms relies on altered expression of endocytic transport regulators (Barbieri et al., 2016; Floyd and De Camilli, 1998; Mellman and Yarden, 2013; Mosesson et al., 2008; Schmid, 2017). The advent of next-generation sequencing technologies has permitted unbiased screening of components orchestrating receptor transport and endo-lysosomal degradation in distinct pathological settings (Buser et al., 2019; Yoshida et al., 2010). Here, by mining TCGA data we revealed differential expression of 113 endocytic machinery components across stages of CRC, several of which were previously shown to be reduced in adenocarcinoma (Kwong et al., 2005; Szymanska et al., 2020; Tanigawa et al., 2019). In-depth validation of our in silico screen uncovered reduced mRNA and protein abundance of $V P S 37 A$ and $V P S 37 B$ paralogs in CRC patients. Our in vitro data further allowed us to propose that Vps37 proteins have partly non-overlapping functions in the cell. We also showed that co-depletion of Vps37 proteins evokes stress responses manifested among others by activation of the NF- $\mathrm{B}$ inflammatory response and $\mathrm{p} 21$-mediated impairment of cell growth. We finally correlated the magnitude of stress responses with the degree of ESCRT-I subunit destabilization after (co-)depletion of $\mathrm{Vps} 37$ proteins. While decreased abundance of $V P S 37 A$ and/or $V P S 37 B$ mRNA and proteins appears not to be an oncogenic driver per se, these passenger alterations might represent potential vulnerabilities of cancer cells to therapeutic treatment.

In humans, there are four VPS37 paralogs with distinct chromosomal localization, number of splicing isoforms, and protein sequence identity. VPS37A, VPS37B, VPS37C and VPS37D are localized on chromosome 8p, 12q, 11q, and 7q, respectively. This different chromosomal localization of VPS37 genes could favor independent regulatory mechanisms of expression in patho-physiological circumstances. Another layer of complexity is added by the incorporation of Vps37 proteins into ESCRT-I to form functionally distinct complexes in the cell (Stefani et al., 2011; Wunderley et al., 2014). Changes in mRNA and protein levels of VPS37A were previously documented in various cancer types, including liver, prostate, breast, ovarian, renal, lung, glioma, gastric, oral and oropharyngeal squamous cell carcinoma and colon cancer (Chen et al., 2015; Chen et al., 2020; Chen et al., 2018; Du et al., 2016; Fu et al., 2018; Lai et al., 2009; Perisanidis et al., 2013; Sun et al., 2017; Vasaikar et al., 2019; Wittinger et al., 2011; Wu et al., 
2019; Xu et al., 2017a; Xu et al., 2017b; Xu et al., 2014; Xu et al., 2017c; Xu et al., 2003; Yang et al., 2016; Yang et al., 2017; Zhu et al., 2015). Several of these studies suggested that VPS37A acts as a tumor suppressor and its loss can serve as an adverse prognostic factor. However, the abundance of the remaining VPS37 paralogs at mRNA and protein levels as well as their contribution to oncogenesis have not been investigated across cancer types and disease stages.

By mining the TCGA expression data, we revealed decreased expression of $V P S 37 B$ during the transition from early to advanced stages of colorectal adenocarcinoma. Analysis of an independent cohort of patients corroborated results of our in silico prediction that VPS37B expression is indeed reduced in adenocarcinoma patients. Additionally, we found decreased expression of VPS37A in the analyzed group of patients. We further confirmed the lower abundance of both proteins in treatment-naïve primary CRC samples belonging largely to the locally advanced disease. The observations we made on VPS37 paralog expression extend previous bioinformatics analysis of COAD and READ cohorts performed without grouping these patients based on disease stages (Miller et al., 2018) and a proteogenomic study of a homogenous cohort of treatment-naïve patients undergoing primary surgery for colon adenocarcinoma (Vasaikar et al., 2019). The VPS37A gene undergoes frequent deletion as a part of $8 \mathrm{p}$ during progression from adenoma to adenocarcinoma, which would explain its decreased abundance at mRNA and protein levels (Meijer et al., 1998). On the other hand, further studies will need to clarify the molecular origin of diminished VPS37B expression in CRC as chromosome 12q, where it is located, undergoes frequent amplifications, suggesting an existence of a (post-)transcriptional mechanism (Wood et al., 2007).

The present study provided comprehensive understanding of the consequences of Vps37 protein depletion in CRC cells in all possible combinations of paralogs expressed at high levels in these cells, namely $V P S 37 A, V P S 37 B$ and VPS37C. Analysis of the transcriptome of CRC cells revealed that Vps37 proteins have partly non-overlapping function as deduced from distinct sets of genes induced upon their individual depletion. It also suggests that the type and magnitude of transcriptional responses upon concurrent VPS37 paralog silencing stem from the cumulative inhibition of cellular processes executed by their protein products. Among genes induced upon either $V P S 37 A B$ or $V P S 37 A B C$ knockdown we did not find prototypical drivers of tumorigenesis but rather cell growth inhibitors, such as $C D K N 1 A / \mathrm{p} 21, C D K N 2 B / \mathrm{p} 14^{\mathrm{INK} 4 \mathrm{~B}}$, and 
$C D K N 2 D / p 19^{\mathrm{INK} 4 \mathrm{D}}$. As a consequence we observed decreased ability of CRC cells to progress over cell cycle phases that resulted in the inhibition of proliferation and colony forming ability. Our results from Vps37ABC-depleted cells reinforce the notion that knockdown of other ESCRT-I components, such as TSG101, VPS28 and UBAP1, induces cell cycle arrest and halts cell proliferation (Krempler et al., 2002; Miller et al., 2018; Morita et al., 2007). Here, we also demonstrate that the degree of cell growth impairment depends primarily on the perturbed expression of $V P S 37 A$ and whether it is silenced on its own or in combination with other paralogs. To our knowledge, expression of neither $V P S 37 B$ nor $V P S 37 C$ has been related to cancer growth but rather to virus release and infectivity (Stuchell et al., 2004). A vast majority of CRC cell lines tested within the DepMap project (Behan et al., 2019) showed no or only slight changes in cell fitness upon RNAi-mediated depletion of VPS37A or VPS37B (VPS37C has not been tested with this technology). Noteworthy, CRISPR-Cas9-mediated knockout of VPS37A markedly decreased RKO and DLD1 cell fitness, whilst the effects of $V P S 37 B$ and $V P S 37 C$ were less deleterious. Observations made within the DepMap project support the results of our colony formation and proliferation assays and point to distinct effects of long- and short-term loss of VPS37 paralogs. Although our data suggest the negative impact of Vps37 paralogs (co-)depletion on cancer cell growth, we postulate that the effect of their CRC-associated reduction is more nuanced. Previous studies revealed that loss of chromosome $8 \mathrm{p}$, where $V P S 37 A$ is located, promotes tumor growth (Cai et al., 2016; Xue et al., 2012). Decreased expression of VPS37 paralogs may be particularly beneficial for cells of advanced stage CRC as RNAi-mediated silencing of VPS37A promoted the resistance of prostate and breast cancer cells to chemotherapeutics (Sun et al., 2017; Yang et al., 2016). Our interrogation of TCGA expression data did not reveal increased transcription of $C D K N 1 A, C D K N 2 B$ and $C D K N 2 D$ in the advanced stages of CRC patients. Notably, this cohort displayed only reduced expression of VPS37B but not VPS37A, which at least in part might explain lack of changes in $C D K N$ expression (Table S6). Thus, we postulate that the impact of VPS37 paralog loss on cancer cell growth warrants further studies in a subset of patients with decreased expression of both VPS37A and VPS37B.

The pronounced inhibition of cell growth in vitro upon Vps37AB and Vps37ABC depletion correlated with activation of inflammatory and stress signaling mediated by NF- $\mathrm{B}$ and MAPK. These cellular responses induced upon silencing of VPS37 paralogs can be viewed as another 
example of sterile inflammation which resembles stress reactions caused by intracellular dysfunction of numerous membrane organelles, such as malfunctioning mitochondria, ER or endosomes (Keestra-Gounder et al., 2016; Maminska et al., 2016; West et al., 2015). Although Vps37B depletion did not promote inflammatory gene transcription in CRC cell lines, the subset of advanced stage CRC patients in the TCGA dataset with decreased VPS37B expression showed elevated mRNA abundance of CXCL8 and ICAM1 (Table S6). In our in vitro experimental setting, expression of these genes was increased only upon concurrent knockdown of either $V P S 37 A B$ or $V P S 37 A B C$. If a subgroup of CRC patients with loss of both $V P S 37 A$ and $V P S 37 B$ was identified, it would be worth testing whether they display an inflammatory phenotype that could be modulated pharmacologically. However, inflammatory gene expression in advanced stage CRC patients is likely a result of multiple lesions accumulated in the course of disease progression. In addition to the expression of several NF- $\kappa \mathrm{B}-d e p e n d e n t$ cytokines that clustered to processes related to (chemo-)taxis of immune system cells, $V P S 37 A B$ and $V P S 37 A B C$ knockdown cells produced high levels of the cell cycle inhibitor p21. Although several papers described $\mathrm{NF}-\kappa \mathrm{B}$ as a regulator of $C D K N 1 A / \mathrm{p} 21$ expression leading to cell cycle arrest in normal and cancer cells (Basile et al., 2003; Hinata et al., 2003; Nicolae et al., 2018; Wuerzberger-Davis et al., 2005), our data point to a different mechanism. Plausibly, it involves the release of Tsg101-mediated repression of the CDKN1A promoter (Lin et al., 2013), which in our study correlated with ESCRT-I destabilization after concurrent depletion of Vps37 proteins. Alternatively, ESCRT-I destabilization upon co-depletion of Vps37 proteins might induce p53driven transcription of CDKN1A (El-Deiry, 2003). On the other hand, the p21 protein was shown to regulate the NF-kB pathway in macrophages (Rackov et al., 2016; Trakala et al., 2009), but we excluded that a similar mechanism occurs in CRC cells. Thus, we concluded that inflammatory response induction and inhibition of cell growth after concurrent VPS37 paralog silencing are two independent and parallel processes.

The most important finding of this study is that differential depletion of Vps37 proteins elicits distinct effects on ESCRT-I subunit stability which align with the type and magnitude of transcriptional responses. Our data reinforce the notion that Vps37 proteins dictate the incorporation of UBAP-1, Mvb12A, and Mvb12B leading to the assembly of distinct ESCRT-I complexes, which could be functionally non-redundant. More specifically, our data indicate a 
partnering preference of Vps37A for UBAP-1 and Vps37B for Mvb12A. This is in line with previous studies on the ESCRT-I stability, which contradicted the stochastic association of ESCRT-I components (Wunderley et al., 2014). We extended these studies showing that concurrent knockdown of all VPS37 paralogs leads to the nearly complete destabilization of remaining ESCRT-I subunits, resembling effects achieved upon either TSG101 or VPS28 silencing. It also explains why we observe similar effects on cell homeostasis, namely induction of inflammatory response and cell growth inhibition, upon knockdown of TSG101 and concurrent silencing of VPS37 paralogs and overall similarities in transcriptional responses (Brankatschk et al., 2012; Maminska et al., 2016; Miller et al., 2018). The degree of ESCRT-I destabilization after (co-)silencing of VPS37 paralogs correlates with the type and magnitude of transcriptional responses; however, the precise mechanism remains to be determined. Destabilization of Vps37 proteins has been well documented upon Tsg101 depletion (Bache et al., 2004; Stefani et al., 2011; Stuchell et al., 2004; Wunderley et al., 2014). Here, we observed mild but distinct transcriptional alterations after either Vps37B or Vps37C depletion that may result from their partly overlapping functions. Both proteins possess the PRR domain and may share similar binding partners. Indeed, using the BioGRID database (Oughtred et al., 2019) we found that both $\mathrm{Vps} 37 \mathrm{~B}$ and $\mathrm{Vps} 37 \mathrm{C}$ have large and partially overlapping interactomes, whilst Vps37A bereft of PRR has only a few interacting proteins. Thus, VPS37A as the only paralog encoding the UEV domain might execute functions that cannot be taken over by any other family member. As a consequence, its depletion induces a distinct set of genes than those expressed after knockdown of either $V P S 37 B$ or $V P S 37 C$. Noteworthy, at least under some conditions, the cell can very well compensate for the loss of a single VPS37 paralog as illustrated by our RNASeq analysis. On the other hand, concurrent silencing of either $V P S 37 A B$ or $V P S 37 A B C$ evokes profound transcriptional alterations that we believe, by similarity to Tsg101 or Vps28 depletion, arise from adverse effects of non-degraded plasma membrane proteins and alterations in protein networks that may contribute to prolonged oncogenic signaling (Maminska et al., 2016). In the context of cancer, ESCRT-I destabilization after concurrent Vps37 depletion could result in more adverse tumor phenotype. This notion is consistent with our transcriptomic analysis of CRC cells depleted of Vps37 proteins, which identified processes related to cell migration, growth and signaling. Downregulation of ESCRT-I components was shown in vitro to prolong epidermal 
580 growth factor signaling, sensitize cells to low doses of transforming growth factor, as well as 581 promote cell invasion and migration through the process of epithelial to mesenchymal transition 582 (Miller et al., 2018; Yang et al., 2016).

583 In summary, we established that ESCRT-I subunit destabilization after co-depletion of Vps37 584 proteins evokes profound cellular stress manifested by a sterile inflammatory response and cell 585 growth arrest. Our findings also revealed potential vulnerabilities of CRC cells with reduced 586 levels of $V P S 37 A$ and $V P S 37 B$ that may be more susceptible to chemotherapeutics and 587 pharmacological modulators of inflammatory response. We also identified candidates with 588 known functions in endocytosis, beyond VPS37 paralogs, whose expression is changed in CRC 589 and thus warrants further investigation in the context of cancer cell pathophysiology.

\section{MATERIAL AND METHODS}

593 Human DLD1 (CCL-221) and RKO (CRL-2577) cell lines were obtained from American Type 594 Culture Collection (ATCC). DLD1 were cultured in Dulbecco's modified Eagle's medium 595 (DMEM, Sigma-Aldrich, M2279) supplemented with 10\% (v/v) fetal bovine serum (FBS, 596 Sigma-Aldrich, F7524) and 2 mM L-Glutamine (Sigma-Aldrich, G7513). RKO were maintained 597 in Eagle's minimum essential medium (EMEM, ATCC, 30-2003) supplemented with 10\% (v/v) 598 FBS. Both cell lines were passaged using 0.05\% Trypsin+EDTA (Sigma-Aldrich, T4049). Cells 599 were cultured in an incubator at $37^{\circ} \mathrm{C}$ in a humidified atmosphere containing $5 \% \mathrm{CO}_{2}$. During 600 the study, cells were regularly tested for mycoplasma and the identities of DLD1 and RKO were 601 confirmed by short tandem repeat (STR) profiling performed by the ATCC Cell Authentication 602 Service.

\section{Cell transfection}

605 Cells were either forward- or reverse-transfected with siRNAs using Lipofectamine RNAiMAX 606 transfection reagent according to the manufacturer's instructions (Thermo Fisher Scientific, 607 13778150). The concentration of single siRNA duplex used for transfection was $20 \mathrm{nM}$. In 
608

609

610

611

612

613

614

615

616

617

618

619

620

621

622

623

624

625

626

627

628

629

630

631

632

633

634

635

636

experiments with simultaneous knockdown of two, three, and four genes, the total concentration of siRNA was $40 \mathrm{nM}, 60 \mathrm{nM}$, and $80 \mathrm{nM}$, respectively and the proportion of individual siRNAs duplexes was kept equal. The following PreDesigned or Validated Ambion Silencer Select siRNAs (Thermo Fisher Scientific) were used: Negative Control No. 1 (siNC\#1, 4390843) and Negative Control No. 2 (siNC\#2, 4390846); on-target siVPS37A\#1 (s44037), siVPS37A\#2 (s44038), siVPS37B\#1 (s36177), siVPS37B\#2 (s36178), siVPS37C\#1 (s30059), siVPS37C\#2 (s30060), siRELA (s11916), siCHUK (s3066), siIKBKB (s7263), siCDKN1A\#1 (s415), siCDKN1A\#2 (s417), siTSG101\#1 (s14439), siTSG101\#2 (s14440), siVPS28\#1 (s27577), siVPS28\#2 (s27579), siUBAP1\#1 (s27812), siUBAP1\#2 (s27813), siMVB12A\#1 (s41121), siMVB12A\#2 (s41122), siMVB12B\#1 (s40157), siMVB12B\#2 (s40158). Additionally, two custom-ordered Silencer Select duplexes were used: Negative Control No. 3 (NC3, sense strand 5'->3' UACGACCGGUCUAUCGUAGtt, antisense strand 5'->3' CUACGAUAGACCGGUCGUAtt) and Negative Control No. 4 (NC4, sense strand 5'->3' UUCUCCGAACGUGUCACGUtt, antisense strand 5'->3' ACGUGACACGUUCGGAGAAtt). The composition of siRNA mixes in experiments with individual and concurrent gene silencing is listed in Table S7.

\section{Transcriptome analysis by RNA sequencing (RNA-Seq)}

Cells were plated in 12-well plate format at the density of 60,000 cells $/ \mathrm{ml}$ in 1 of medium. After 16-24 $\mathrm{h}$ cells were left non-transfected or differentially transfected according to the forward transfection protocol. $72 \mathrm{~h}$ later, cells were washed with PBS, and the cell pellet was collected. Sequencing libraries were generated using Ion AmpliSeq Transcriptome Human Gene Expression Panel (ThermoFisher Scientific). Sequencing was performed using Ion Proton instrument with 7 or 8 samples per chip with Ion PI Hi-Q Sequencing 200 Kit (ThermoFisher Scientific). Reads were aligned to the hg19 AmpliSeq Transcriptome ERCC v1 with Torrent Mapping Alignment Program (version 5.0.4, ThermoFisher Scientific). Transcripts were quantified with HTseq-count (version 0.6.0) run with default settings (Anders et al., 2015).

Gene level differential expression analysis was performed using the R package DESeq2 (version 1.18.1; (Love et al., 2014)) for genes with at least 100 counts across conditions and by taking 
into the account the batch effect and applying the following contrasts $(\alpha=0.05)$ : NT (nontransfected) versus siCTRL\#1, NT versus siVPS37A\#1, NT versus siVPS37B\#1, NT versus siVPS37C\#1, NT versus siVPS37AB\#1, NT versus siVPS37AC\#1, NT versus siVPS37BC\#1, NT versus siVPS37ABC\#1, siCTRL\#1-T versus siVPS37A\#1, siCTRL\#1-T versus siVPS37B\#1, siCTRL\#1-T versus siVPS37C\#1, siCTRL\#1-T versus siVPS37AB\#1, siCTRL\#1$\mathrm{T}$ versus siVPS37AC\#1, siCTRL\#1-T versus siVPS37BC\#1, siCTRL\#1-T versus siVPS37ABC\#1. We excluded non-protein coding genes from downstream analysis.

The overlap for different silencing conditions and normalization contrasts was visualized using the VennDiagram package (version 1.6.20). The genes, which overlapped for on-target siRNAs normalized against either NT or siCTRL\#1- transfected patterns, were subjected to GO analysis of biological processes and Reactome pathway analysis using clusterProfiler (version 3.6.0; (Yu et al., 2012)) and ReactomePA R-packages (version 3.8; (Yu and He, 2016)) taking advantage of enrichGO and enrichPathway functions, respectively. All enrichment p-values in GO analysis were corrected for multiple testing using the Benjamini-Hochberg method and only genes with adjusted p-value $<0.05$ were considered significant. The minimal and maximal sizes of gene clusters were set to 10 and 500, respectively. Redundant terms were removed by means of the simplify function with cutoff 0.6. Count data were transformed using the Transcript Per Million (TPM) and scaled across conditions (Z-score). Differentially expressed genes binned in the selected GO processes were used for hierarchical clustering, which was performed on Euclidean distances using Ward's algorithm. Heatmaps of differentially expressed genes were visualized using ComplexHeatmap (version 1.17.1; (Gu et al., 2016)). All calculations were performed in R version 3.4.4 (https://www.R- project.org).

The code for the present analysis is available on GitHub (https://github.com/kkolmus/VPS37_RNA-Seq). RNA-Seq data have been deposited at Gene Expression Omnibus (GEO) under the accession code: GSE152195.

\section{Clonogenic assay}

Non-transfected cells or cells subjected to reverse transfection with different siRNAs were seeded at the density of 1000 cells per well in a 6-well plate format and cultured for 14 days to 
666 form colonies. For staining, colonies were washed with PBS, fixed for 5 min in a 3:1 (v/v)

667

668

669

670

671

672

673

674

675

676

677

678

679

680

681

682

683

684

685

686

687

688

689

690

691

692

693

694

solution of acetic acid:methanol, and incubated for $15 \mathrm{~min}$ in $0.2 \%$ crystal violet solution in $70 \%$ ethanol. The whole procedure was performed at room temperature. Plates with colonies were scanned using the Odyssey Infrared System (LI-COR, Biosciences). Acquired images were analyzed as described before (Guzman et al., 2014). Data are expressed as the percentage staining intensity displayed by non-transfected cells.

\section{Proliferation assay}

1500 cells were left non-transfected or reverse-transfected with different siRNAs in a 96-well plate format and let proliferate for $120 \mathrm{~h}$. BrdU Cell Proliferation ELISA assay (Roche, 11647229001) was used to assess the proliferation of RKO and DLD1 cells according to the manufacturer's instructions with the following modifications: BrdU reagent was added $4 \mathrm{~h}$ prior to cell fixation, $100 \mu \mathrm{l}$ of substrate solution was added for $5 \mathrm{~min}$ followed by addition of $50 \mu \mathrm{l}$ of $1 \mathrm{M} \mathrm{HCl}$. The colorimetric signal was detected at $450 \mathrm{~nm}$ using the Tecan Sunrise Microplate Reader system with the Magellan v. 6.6 software. Data are expressed as the percentage of proliferating, non-transfected cells.

\section{Western blotting and densitometry analysis}

Cells were plated in either 6- or 12-well plate format at the density of 60,000 cells $/ \mathrm{ml}$ in 1 and 2 $\mathrm{ml}$ of medium, respectively. After 16-24 h cells were left non-transfected or differentially transfected according to the forward transfection protocol. $72 \mathrm{~h}$ later, cells were lysed in RIPA buffer (1\% Triton X-100, 0.5\% sodium deoxycholate, 0.1\% SDS, $50 \mathrm{mM}$ Tris $\mathrm{pH} 7.4,150 \mathrm{mM}$ $\mathrm{NaCl}, 0.5 \mathrm{mM}$ EDTA) supplemented with protease inhibitor cocktail $(6 \mu \mathrm{g} / \mathrm{ml}$ chemostatin, 0.5 $\mu \mathrm{g} / \mathrm{ml}$ leupeptin, $10 \mu \mathrm{g} / \mathrm{ml}$ antipain, $2 \mu \mathrm{g} / \mathrm{ml}$ aprotinin, $0.7 \mu \mathrm{g} / \mathrm{ml}$ pepstatin $\mathrm{A}, 10 \mu \mathrm{g} / \mathrm{ml}$ 4amidinophenylmethanesulfonyl fluoride hydrochloride; Sigma-Aldrich) and phosphatase inhibitor cocktails 2 and 3 (P5726 and P0044, Sigma-Aldrich). Protein concentration was determined using the BCA Protein Assay Kit (Thermo Fisher Scientific, 23225). 25-30 $\mu \mathrm{g}$ of total protein per sample was resolved on 12 or $15 \%$ SDS-PAGE, transferred onto a nitrocellulose membrane (Amersham Hybond, GE Healthcare Life Science, 10600002), blocked with 5\% milk 
695

696

697

698

699

700

701

702

703

704

705

706

707

708

709

710

711

712

713

714

715

716

717

718

719

720

721

722

in PBS with 1\% Tween, probed first with specific primary and then secondary antibodies, and imaged using the detection solution (BioRad, 170-5061) and ChemiDoc imaging system (BioRad). All primary antibodies are listed in Table S8. Secondary horseradish peroxidaseconjugated anti-mouse (315-005-008), anti-rabbit (111-035-144) and anti-goat (305-035-046) antibodies were from Jackson ImmunoResearch and were used at working dilution 1:10,000. Densitometry of protein bands was carried out using ImageJ software (Schneider et al., 2012). p65 was used as the loading control for quantification of phosphorylated p65. Vinculin was used as a loading control in all other experiments. Results are presented as fold change compared to non-transfected cells.

\section{Quantitative Real-Time Polymerase Chain Reaction (qRT-PCR)}

Cells were seeded in a 12-well plate format at the density of 60,000 cells $/ \mathrm{ml}$ in $1 \mathrm{ml}$ of medium. After 16-24 h cells were left non-transfected or differentially transfected according to the forward transfection protocol. $72 \mathrm{~h}$ later, total RNA was isolated with High Pure Isolation Kit (Roche, 11828665001). $500 \mathrm{ng}$ of total RNA was subjected for cDNA synthesis. M-MLV, random nonamers and oligo(dT) $)_{23}$ (Sigma-Aldrich, M1302, R7647, and O4387, respectively) were used for cDNA synthesis according to the manufacturer's instructions. Expression of genes of interest was measured using primers designed with the NCBI Primer designing tool and custom-synthesized by Sigma-Aldrich. We list primers used in the present study (Table S9). Real-Time cDNA amplification was performed with the Kapa Sybr Fast qPCR Kit (KapaBiosystems, KK4618). Fluorescence was monitored using the 7900HT Fast Real-Time PCR thermocycler (Applied Biosystems). Expression of each gene was normalized to either expression of the $A C T B$ ( $\beta$-actin) or GAPDH (glyceraldehyde 3-phosphate dehydrogenase) reference genes. Results are presented as fold change compared to non-transfected cells. For clarity, the Y-axis is interrupted in some cases.

\section{Analysis of expression levels of VPS37 paralogs in healthy and colorectal cancer (CRC) samples using qRT-PCR}


Samples of the normal colon $(n=24)$ and adenocarcinoma $(n=26)$ had been collected for the purpose of previous studies (Mikula et al., 2011; Skrzypczak et al., 2010). In order to determine the abundance of VPS37A and VPS37B transcripts, qRT-PCR was performed as described before (Mikula et al., 2011; Skrzypczak et al., 2010). The sequences of primers for VPS37A and $V P S 37 B$ are listed in Table S9.

\section{Flow cytometry analysis}

Cells were seeded in a 6-well plate format at the density of 60,000 cells $/ \mathrm{ml}$ in $2 \mathrm{ml}$ of medium. After 16-24 h cells were left non-transfected or differentially transfected according to the forward transfection protocol. $96 \mathrm{~h}$ post-transfection cells were briefly washed with PBS, harvested with trypsin+EDTA, washed twice with PBS, and fixed for $24 \mathrm{~h}$ in ice-cold $70 \%$ ethanol. Washed cells were then incubated first with extraction buffer $(4 \mathrm{mM}$ citric acid in $0.2 \mathrm{M}$ $\mathrm{Na}_{2} \mathrm{HPO}_{4}$ ) for $5 \mathrm{~min}$ at room temperature, and next with staining solution $(3.8 \mathrm{mM}$ sodium citrate, $50 \mu \mathrm{g} / \mathrm{ml}$ propidium iodide (PI) and $0.5 \mathrm{mg} / \mathrm{ml}$ RNase A) for $30 \mathrm{~min}$ at room temperature. Analysis of cells was performed on the BD LSRFortessa flow cytometer (Bekton Dickinson). A total of 10,000 cells from single cell gate were counted for each transfection condition. Flow cytometry data were plotted and analyzed by FlowJo (Bekton Dickinson) and ModFit LT (Verity Software House) software. Data is presented as percentage of analyzed cells in the given cell cycle phase.

\section{Immunohistochemistry (IHC) and analyses of normal and CRC samples}

The study protocol for analysis of protein levels of Vps37A and Vps37B in human normal colon and CRC samples was approved by the Bioethics Committee of the Maria Skłodowska-Curie National Research Institute of Oncology in Warsaw (decision no. 40/2017). Informed consent was obtained from all subjects. The experiment conformed to the principles set out in the WMA Declaration of Helsinki and the Department of Health and Human Services Belmont Report. High-density tissue microarrays were constructed from formalin-fixed, paraffin-embedded diagnostic samples of 100 pairs of treatment-naïve CRC tissues and matched normal colon samples from the collection of the Maria Skłodowska-Curie National Research Institute of 
752 Oncology in Warsaw. IHC was performed using automated immunohistochemical stainer (Dako

\section{TCGA data analysis}

Clinicopathological and transcriptional profiles from the two TCGA cohorts: rectum adenocarcinoma (READ) and colon adenocarcinoma (COAD) were retrieved using the TCGAbiolinks package (Colaprico et al., 2016). READ and COAD datasets were analyzed together as a previous study showed a major overlap in their expression patterns (Weinstein et al., 2013). The present analysis encompassed only matched normal-tumor tissue samples for which clinicopathological data were available. 31 patients fulfilled the latter criterion. 19 of these patients were assigned to the early stage group encompassing stages I and II, and 12 were assigned to the advanced stage group encompassing stages III and IV. Matching TCGA sequencing data acquired using the Illumina HiSeq platform were used. Differential gene expression was performed with the default settings on count data taking into account correction of batch effect relying on the inter-plate variation. Only transcripts whose gene counts exceeded 50 were selected for downstream analysis. Volcano plots of differentially expressed genes were prepared with ggplot2 (CRAN available package). Heatmaps of differentially expressed genes were visualized using ComplexHeatmap (version 1.17.1; (Gu et al., 2016)). The overlap between differentially expressed genes was visualized using the VennDigram package (CRAN available package). All calculations were performed in R version 3.6.1 (https://www.R-project.org). The code for the present analysis is available on GitHub (https://github.com/kkolmus/TCGA_transcriptomic_proj).

\section{Statistical analysis}


781 Data are shown as mean \pm standard deviation of at least three independent biological 782 experiments. For qRT-PCR and BrdU, samples were assayed in technical triplicates. Statistical 783 analysis was performed using the GraphPad Prism 6 software. Experiments with normal 784 distribution were analyzed using either Student's t-test or one-way ANOVA followed by 785 Bonferroni's multiple comparison test. In the case of non-normal distribution Mann-Whitney U786 test was applied. Analysis of categorical data was performed using Fisher's exact test. The 787 significance of mean comparison is annotated as follows: ns, non-significant $(P \geq 0.05),{ }^{*} P<0.05$, $788 * * P<0.01, * * * P<0.001, * * * * P<0.0001$. Results were considered significant when $P<0.05$. No 789 statistical methods were used to predetermine sample size. 


\section{References}

Aibar, S., Gonzalez-Blas, C. B., Moerman, T., Huynh-Thu, V. A., Imrichova, H., Hulselmans, G., Rambow, F., Marine, J. C., Geurts, P., Aerts, J. et al. (2017). SCENIC: single-cell regulatory network inference and clustering. Nat Methods 14, 1083-1086.

Alfred, V. and Vaccari, T. (2016). When membranes need an ESCRT: endosomal sorting and membrane remodelling in health and disease. Swiss Med Wkly 146, w14347.

Anders, S., Pyl, P. T. and Huber, W. (2015). HTSeq--a Python framework to work with high-throughput sequencing data. Bioinformatics 31, 166-9.

Bache, K. G., Slagsvold, T., Cabezas, A., Rosendal, K. R., Raiborg, C. and Stenmark, H. (2004). The growth-regulatory protein HCRP1/hVps37A is a subunit of mammalian ESCRT-I and mediates receptor down-regulation. Mol Biol Cell 15, 4337-46.

\section{Banach-Orlowska, M., Jastrzebski, K., Cendrowski, J., Maksymowicz, M.,} Wojciechowska, K., Korostynski, M., Moreau, D., Gruenberg, J. and Miaczynska, M. (2018). The topology of the lymphotoxin beta receptor that accumulates upon endolysosomal dysfunction dictates the NF-kappaB signaling outcome. J Cell Sci 131.

Barbieri, E., Di Fiore, P. P. and Sigismund, S. (2016). Endocytic control of signaling at the plasma membrane. Curr Opin Cell Biol 39, 21-7.

Basile, J. R., Eichten, A., Zacny, V. and Munger, K. (2003). NF-kappaB-mediated induction of $\mathrm{p} 21(\mathrm{Cip} 1 / \mathrm{Waf1})$ by tumor necrosis factor alpha induces growth arrest and cytoprotection in normal human keratinocytes. Mol Cancer Res 1, 262-70.

Behan, F. M., Iorio, F., Picco, G., Goncalves, E., Beaver, C. M., Migliardi, G., Santos, R., Rao, Y., Sassi, F., Pinnelli, M. et al. (2019). Prioritization of cancer therapeutic targets using CRISPR-Cas9 screens. Nature 568, 511-516.

Bishop, N., Horman, A. and Woodman, P. (2002). Mammalian class E vps proteins recognize ubiquitin and act in the removal of endosomal protein-ubiquitin conjugates. $J$ Cell Biol 157, 91-101. 
Bonelli, P., Tuccillo, F. M., Borrelli, A., Schiattarella, A. and Buonaguro, F. M. (2014). CDK/CCN and CDKI alterations for cancer prognosis and therapeutic predictivity. Biomed Res Int 2014, 361020.

Brankatschk, B., Wichert, S. P., Johnson, S. D., Schaad, O., Rossner, M. J. and Gruenberg, J. (2012). Regulation of the EGF transcriptional response by endocytic sorting. Sci Signal 5, ra21.

Buser, D. P., Ritz, M. F., Moes, S., Tostado, C., Frank, S., Spiess, M., Mariani, L., Jeno, P., Boulay, J. L. and Hutter, G. (2019). Quantitative proteomics reveals reduction of endocytic machinery components in gliomas. EBioMedicine 46, 32-41.

Cai, Y., Crowther, J., Pastor, T., Abbasi Asbagh, L., Baietti, M. F., De Troyer, M., Vazquez, I., Talebi, A., Renzi, F., Dehairs, J. et al. (2016). Loss of Chromosome 8p Governs Tumor Progression and Drug Response by Altering Lipid Metabolism. Cancer Cell 29, 751-766.

Chen, F., Deng, J., Liu, X., Li, W. and Zheng, J. (2015). HCRP-1 regulates cell migration and invasion via EGFR-ERK mediated up-regulation of MMP-2 with prognostic significance in human renal cell carcinoma. Sci Rep 5, 13470.

Chen, F., Wu, J., Teng, J., Li, W., Zheng, J. and Bai, J. (2020). HCRP-1 regulates cell migration, invasion and angiogenesis via Src/ FAK signaling in human prostate cancer. Int J Biol Sci 16, 342-352.

Chen, F., Zhang, L., Wu, J., Huo, F., Ren, X., Zheng, J. and Pei, D. (2018). HCRP-1 regulates EGFR-AKT-BIM-mediated anoikis resistance and serves as a prognostic marker in human colon cancer. Cell Death Dis 9, 1176.

Colaprico, A., Silva, T. C., Olsen, C., Garofano, L., Cava, C., Garolini, D., Sabedot, T. S., Malta, T. M., Pagnotta, S. M., Castiglioni, I. et al. (2016). TCGAbiolinks: an R/Bioconductor package for integrative analysis of TCGA data. Nucleic Acids Res 44, e71.

Di Fiore, P. P. and von Zastrow, M. (2014). Endocytosis, signaling, and beyond. Cold Spring Harb Perspect Biol 6.

Dou, Y., Cha, D. J., Franklin, J. L., Higginbotham, J. N., Jeppesen, D. K., Weaver, A. M., Prasad, N., Levy, S., Coffey, R. J., Patton, J. G. et al. (2016). Circular RNAs are down- 
844

845

846

847

848

849

850

851

852

853

854

855

856

857

858

859

860

861

862

863

864

865

866

867

868

869

870

regulated in KRAS mutant colon cancer cells and can be transferred to exosomes. Sci Rep 6, 37982.

Du, Y., Wang, P., Sun, H., Yang, J., Lang, X., Wang, Z., Zang, S., Chen, L., Ma, J. and Sun, D. (2016). HCRP1 is downregulated in non-small cell lung cancer and regulates proliferation, invasion, and drug resistance. Tumour Biol.

El-Deiry, W. S. (2003). The role of p53 in chemosensitivity and radiosensitivity.

Oncogene 22, 7486-95.

Floyd, S. and De Camilli, P. (1998). Endocytosis proteins and cancer: a potential link? Trends Cell Biol 8, 299-301.

Fu, F., Wan, X., Wang, D., Kong, Z., Zhang, Y., Huang, W., Wang, C., Wu, H. and Li, Y. (2018). MicroRNA-19a acts as a prognostic marker and promotes prostate cancer progression via inhibiting VPS37A expression. Oncotarget 9, 1931-1943.

Gargalionis, A. N., Karamouzis, M. V., Adamopoulos, C. and Papavassiliou, A. G. (2015). Protein trafficking in colorectal carcinogenesis-targeting and bypassing resistance to currently applied treatments. Carcinogenesis 36, 607-15.

Gingras, M. C., Kazan, J. M. and Pause, A. (2017). Role of ESCRT component HDPTP/PTPN23 in cancer. Biochem Soc Trans 45, 845-854.

Gu, Z., Eils, R. and Schlesner, M. (2016). Complex heatmaps reveal patterns and correlations in multidimensional genomic data. Bioinformatics 32, 2847-9.

Guzman, C., Bagga, M., Kaur, A., Westermarck, J. and Abankwa, D. (2014).

ColonyArea: an ImageJ plugin to automatically quantify colony formation in clonogenic assays. PLoS One 9, e92444.

Hayden, M. S. and Ghosh, S. (2008). Shared principles in NF-kappaB signaling. Cell 132, 344-62.

Hinata, K., Gervin, A. M., Jennifer Zhang, Y. and Khavari, P. A. (2003). Divergent gene regulation and growth effects by NF-kappa B in epithelial and mesenchymal cells of human skin. Oncogene 22, 1955-64. 
Hoesel, B. and Schmid, J. A. (2013). The complexity of NF-kappaB signaling in inflammation and cancer. Mol Cancer 12, 86.

Hurley, J. H. (2015). ESCRTs are everywhere. EMBO J 34, 2398-407.

Keestra-Gounder, A. M., Byndloss, M. X., Seyffert, N., Young, B. M., ChavezArroyo, A., Tsai, A. Y., Cevallos, S. A., Winter, M. G., Pham, O. H., Tiffany, C. R. et al. (2016). NOD1 and NOD2 signalling links ER stress with inflammation. Nature 532, 394-7.

Krempler, A., Henry, M. D., Triplett, A. A. and Wagner, K. U. (2002). Targeted deletion of the Tsg101 gene results in cell cycle arrest at G1/S and p53-independent cell death. $J$ Biol Chem 277, 43216-23.

Kwong, K. Y., Bloom, G. C., Yang, I., Boulware, D., Coppola, D., Haseman, J., Chen, E., McGrath, A., Makusky, A. J., Taylor, J. et al. (2005). Synchronous global assessment of gene and protein expression in colorectal cancer progression. Genomics 86, 14258.

Lai, M. W., Huang, S. F., Lin, S. M., Chen, T. C., Lin, C. Y., Yeh, C. N., Yeh, T. S., Chen, M. F. and Yeh, C. T. (2009). Expression of the HCRP1 mRNA in HCC as an independent predictor of disease-free survival after surgical resection. Hepatol Res 39, 164-76.

Ledoux, A. C. and Perkins, N. D. (2014). NF-kappaB and the cell cycle. Biochem Soc Trans 42, 76-81.

Li, L. and Cohen, S. N. (1996). Tsg101: a novel tumor susceptibility gene isolated by controlled homozygous functional knockout of allelic loci in mammalian cells. Cell 85, 319-29.

Lin, Y. S., Chen, Y. J., Cohen, S. N. and Cheng, T. H. (2013). Identification of TSG101 functional domains and p21 loci required for TSG101-mediated p21 gene regulation. PLoS One 8, e79674.

Love, M. I., Huber, W. and Anders, S. (2014). Moderated estimation of fold change and dispersion for RNA-seq data with DESeq2. Genome Biol 15, 550.

Maminska, A., Bartosik, A., Banach-Orlowska, M., Pilecka, I., Jastrzebski, K., Zdzalik-Bielecka, D., Castanon, I., Poulain, M., Neyen, C., Wolinska-Niziol, L. et al. (2016). 
898

899

900

901

902

903

904

905

906

907

908

909

910

911

912

913

914

915

916

917

918

919

920

921

922

923

924

ESCRT proteins restrict constitutive NF-kappaB signaling by trafficking cytokine receptors. Sci Signal 9, ra8.

Manteghi, S., Gingras, M. C., Kharitidi, D., Galarneau, L., Marques, M., Yan, M., Cencic, R., Robert, F., Paquet, M., Witcher, M. et al. (2016). Haploinsufficiency of the ESCRT Component HD-PTP Predisposes to Cancer. Cell Rep 15, 1893-900.

Mattissek, C. and Teis, D. (2014). The role of the endosomal sorting complexes required for transport (ESCRT) in tumorigenesis. Mol Membr Biol 31, 111-9.

Meijer, G. A., Hermsen, M. A., Baak, J. P., van Diest, P. J., Meuwissen, S. G., Belien, J. A., Hoovers, J. M., Joenje, H., Snijders, P. J. and Walboomers, J. M. (1998).

Progression from colorectal adenoma to carcinoma is associated with non-random chromosomal gains as detected by comparative genomic hybridisation. J Clin Pathol 51, 901-9.

Mellman, I. and Yarden, Y. (2013). Endocytosis and cancer. Cold Spring Harb Perspect Biol 5, a016949.

Mikula, M., Rubel, T., Karczmarski, J., Goryca, K., Dadlez, M. and Ostrowski, J. (2011). Integrating proteomic and transcriptomic high-throughput surveys for search of new biomarkers of colon tumors. Funct Integr Genomics 11, 215-24.

Miller, D. S. J., Bloxham, R. D., Jiang, M., Gori, I., Saunders, R. E., Das, D., Chakravarty, P., Howell, M. and Hill, C. S. (2018). The Dynamics of TGF-beta Signaling Are Dictated by Receptor Trafficking via the ESCRT Machinery. Cell Rep 25, 1841-1855 e5.

Moberg, K. H., Schelble, S., Burdick, S. K. and Hariharan, I. K. (2005). Mutations in erupted, the Drosophila ortholog of mammalian tumor susceptibility gene 101, elicit non-cellautonomous overgrowth. Dev Cell 9, 699-710.

Morita, E., Sandrin, V., Chung, H. Y., Morham, S. G., Gygi, S. P., Rodesch, C. K. and Sundquist, W. I. (2007). Human ESCRT and ALIX proteins interact with proteins of the midbody and function in cytokinesis. EMBO J 26, 4215-27.

Mosesson, Y., Mills, G. B. and Yarden, Y. (2008). Derailed endocytosis: an emerging feature of cancer. Nat Rev Cancer 8, 835-50. 
Nicolae, C. M., O'Connor, M. J., Constantin, D. and Moldovan, G. L. (2018).

NFkappaB regulates p21 expression and controls DNA damage-induced leukemic differentiation. Oncogene 37, 3647-3656.

Olmos, Y. and Carlton, J. G. (2016). The ESCRT machinery: new roles at new holes. Curr Opin Cell Biol 38, 1-11.

Oughtred, R., Stark, C., Breitkreutz, B. J., Rust, J., Boucher, L., Chang, C., Kolas, N., O'Donnell, L., Leung, G., McAdam, R. et al. (2019). The BioGRID interaction database: 2019 update. Nucleic Acids Res 47, D529-D541.

Perisanidis, C., Savarese-Brenner, B., Wurger, T., Wrba, F., Huynh, A., Schopper, C., Kornek, G., Selzer, E., Ewers, R., Psyrri, A. et al. (2013). HCRP1 expression status is a significant prognostic marker in oral and oropharyngeal cancer. Oral Dis 19, 206-11.

Porther, N. and Barbieri, M. A. (2015). The role of endocytic Rab GTPases in regulation of growth factor signaling and the migration and invasion of tumor cells. Small GTPases 6, 135-44.

Rackov, G., Hernandez-Jimenez, E., Shokri, R., Carmona-Rodriguez, L., Manes, S., Alvarez-Mon, M., Lopez-Collazo, E., Martinez, A. C. and Balomenos, D. (2016). p21 mediates macrophage reprogramming through regulation of p50-p50 NF-kappaB and IFN-beta. $J$ Clin Invest 126, 3089-103.

Sadler, J. B. A., Wenzel, D. M., Williams, L. K., Guindo-Martinez, M., Alam, S. L., Mercader, J. M., Torrents, D., Ullman, K. S., Sundquist, W. I. and Martin-Serrano, J. (2018). A cancer-associated polymorphism in ESCRT-III disrupts the abscission checkpoint and promotes genome instability. Proc Natl Acad Sci U S A 115, E8900-E8908.

Schmid, S. L. (2017). Reciprocal regulation of signaling and endocytosis: Implications for the evolving cancer cell. J Cell Biol 216, 2623-2632.

Schneider, C. A., Rasband, W. S. and Eliceiri, K. W. (2012). NIH Image to ImageJ: 25 years of image analysis. Nat Methods 9, 671-5.

Siegel, R. L., Miller, K. D. and Jemal, A. (2018). Cancer statistics, 2018. CA Cancer J Clin 68, 7-30. 
Skrzypczak, M., Goryca, K., Rubel, T., Paziewska, A., Mikula, M., Jarosz, D., Pachlewski, J., Oledzki, J. and Ostrowski, J. (2010). Modeling oncogenic signaling in colon tumors by multidirectional analyses of microarray data directed for maximization of analytical reliability. PLoS One 5.

Stefani, F., Zhang, L., Taylor, S., Donovan, J., Rollinson, S., Doyotte, A., Brownhill, K., Bennion, J., Pickering-Brown, S. and Woodman, P. (2011). UBAP1 is a component of an endosome-specific ESCRT-I complex that is essential for MVB sorting. Curr Biol 21, 1245-50.

Stuchell, M. D., Garrus, J. E., Muller, B., Stray, K. M., Ghaffarian, S., McKinnon, R., Krausslich, H. G., Morham, S. G. and Sundquist, W. I. (2004). The human endosomal sorting complex required for transport (ESCRT-I) and its role in HIV-1 budding. $J$ Biol Chem 279, 36059-71.

Sun, L., Lu, J., Ding, S., Bi, D., Ding, K., Niu, Z. and Liu, P. (2017). HCRP1 regulates proliferation, invasion, and drug resistance via EGFR signaling in prostate cancer. Biomed Pharmacother 91, 202-207.

Szymanska, E., Budick-Harmelin, N. and Miaczynska, M. (2018). Endosomal "sort" of signaling control: The role of ESCRT machinery in regulation of receptor-mediated signaling pathways. Semin Cell Dev Biol 74, 11-20.

Szymanska, E., Nowak, P., Kolmus, K., Cybulska, M., Goryca, K., DerezinskaWolek, E., Szumera-Cieckiewicz, A., Brewinska-Olchowik, M., Grochowska, A., Piwocka, K. et al. (2020). Synthetic lethality between VPS4A and VPS4B triggers an inflammatory response in colorectal cancer. EMBO Mol Med 12, e10812.

Tanigawa, K., Maekawa, M., Kiyoi, T., Nakayama, J., Kitazawa, R., Kitazawa, S., Semba, K., Taguchi, T., Akita, S., Yoshida, M. et al. (2019). SNX9 determines the surface levels of integrin beta1 in vascular endothelial cells: Implication in poor prognosis of human colorectal cancers overexpressing SNX9. J Cell Physiol 234, 17280-17294.

Taniguchi, K. and Karin, M. (2018). NF-kappaB, inflammation, immunity and cancer: coming of age. Nat Rev Immunol 18, 309-324. 
980

981

982

983

984

985

986

987

988

989

990

991

992

993

994

995

996

997

998

999

1000

1001

1002

1003

1004

1005

1006

Tashima, T. (2018). Effective cancer therapy based on selective drug delivery into cells across their membrane using receptor-mediated endocytosis. Bioorg Med Chem Lett 28, 30153024.

Trakala, M., Arias, C. F., Garcia, M. I., Moreno-Ortiz, M. C., Tsilingiri, K., Fernandez, P. J., Mellado, M., Diaz-Meco, M. T., Moscat, J., Serrano, M. et al. (2009). Regulation of macrophage activation and septic shock susceptibility via p21(WAF1/CIP1). Eur J Immunol 39, 810-9.

Vasaikar, S., Huang, C., Wang, X., Petyuk, V. A., Savage, S. R., Wen, B., Dou, Y., Zhang, Y., Shi, Z., Arshad, O. A. et al. (2019). Proteogenomic Analysis of Human Colon Cancer Reveals New Therapeutic Opportunities. Cell 177, 1035-1049 e19.

Vietri, M., Radulovic, M. and Stenmark, H. (2020). The many functions of ESCRTs. Nat Rev Mol Cell Biol 21, 25-42.

Weinstein, J. N., Collisson, E. A., Mills, G. B., Shaw, K. R., Ozenberger, B. A., Ellrott, K., Shmulevich, I., Sander, C. and Stuart, J. M. (2013). The Cancer Genome Atlas Pan-Cancer analysis project. Nat Genet 45, 1113-20.

West, A. P., Khoury-Hanold, W., Staron, M., Tal, M. C., Pineda, C. M., Lang, S. M., Bestwick, M., Duguay, B. A., Raimundo, N., MacDuff, D. A. et al. (2015). Mitochondrial DNA stress primes the antiviral innate immune response. Nature 520, 553-7.

Wittinger, M., Vanhara, P., El-Gazzar, A., Savarese-Brenner, B., Pils, D., Anees, M., Grunt, T. W., Sibilia, M., Holcmann, M., Horvat, R. et al. (2011). hVps37A Status affects prognosis and cetuximab sensitivity in ovarian cancer. Clin Cancer Res 17, 7816-27.

Wood, L. D., Parsons, D. W., Jones, S., Lin, J., Sjoblom, T., Leary, R. J., Shen, D., Boca, S. M., Barber, T., Ptak, J. et al. (2007). The genomic landscapes of human breast and colorectal cancers. Science 318, 1108-13.

Wu, Y., Yang, Y. and Xian, Y. S. (2019). HCRP1 inhibits cell proliferation and invasion and promotes chemosensitivity in esophageal squamous cell carcinoma. Chem Biol Interact $\mathbf{3 0 8}$, 357-363. 
Wuerzberger-Davis, S. M., Chang, P. Y., Berchtold, C. and Miyamoto, S. (2005). Enhanced G2-M arrest by nuclear factor-\{kappa\}B-dependent p21waf1/cip1 induction. Mol Cancer Res 3, 345-53.

Wunderley, L., Brownhill, K., Stefani, F., Tabernero, L. and Woodman, P. (2014). The molecular basis for selective assembly of the UBAP1-containing endosome-specific ESCRT-I complex. J Cell Sci 127, 663-72.

Xu, C. Y., Li, Z. J. and Hu, W. Z. (2017a). Up-regulation of HCRP1 inhibits proliferation and invasion in glioma cells via suppressing the ERK and AKT signaling pathways. Biomed Pharmacother 95, 31-36.

Xu, H., Miao, Z. F., Wang, Z. N., Zhao, T. T., Xu, Y. Y., Song, Y. X., Huang, J. Y., Zhang, J. Y., Liu, X. Y., Wu, J. H. et al. (2017b). HCRP1 downregulation confers poor prognosis and induces chemoresistance through regulation of EGFR-AKT pathway in human gastric cancer. Virchows Arch 471, 743-751.

Xu, J., Yang, W., Wang, Q., Zhang, Q., Li, X., Lin, X., Liu, X. and Qin, Y. (2014). Decreased HCRP1 expression is associated with poor prognosis in breast cancer patients. Int $J$ Clin Exp Pathol 7, 7915-22.

Xu, J., Zhang, X., Wang, H., Ge, S., Gao, T., Song, L., Wang, X., Li, H., Qin, Y. and Zhang, Z. (2017c). HCRP1 downregulation promotes hepatocellular carcinoma cell migration and invasion through the induction of EGFR activation and epithelial-mesenchymal transition. Biomed Pharmacother 88, 421-429.

Xu, Z., Liang, L., Wang, H., Li, T. and Zhao, M. (2003). HCRP1, a novel gene that is downregulated in hepatocellular carcinoma, encodes a growth-inhibitory protein. Biochem Biophys Res Commun 311, 1057-66.

Xue, W., Kitzing, T., Roessler, S., Zuber, J., Krasnitz, A., Schultz, N., Revill, K., Weissmueller, S., Rappaport, A. R., Simon, J. et al. (2012). A cluster of cooperating tumorsuppressor gene candidates in chromosomal deletions. Proc Natl Acad Sci U S A 109, 8212-7. 
Yang, W., Wang, J. G., Wang, Q., Qin, Y., Lin, X., Zhou, D., Ren, K., Hou, C., Xu, J. and Liu, X. (2016). Decreased HCRP1 promotes breast cancer metastasis by enhancing EGFR phosphorylation. Biochem Biophys Res Commun 477, 222-8.

Yang, W., Wang, J. G., Xu, J., Zhou, D., Ren, K., Hou, C., Chen, L. and Liu, X. (2017). HCRP1 inhibits TGF-beta induced epithelial-mesenchymal transition in hepatocellular carcinoma. Int J Oncol.

Yoshida, T., Kobayashi, T., Itoda, M., Muto, T., Miyaguchi, K., Mogushi, K., Shoji, S., Shimokawa, K., Iida, S., Uetake, H. et al. (2010). Clinical omics analysis of colorectal cancer incorporating copy number aberrations and gene expression data. Cancer Inform 9, 14761.

Yu, G. and He, Q. Y. (2016). ReactomePA: an R/Bioconductor package for reactome pathway analysis and visualization. Mol Biosyst 12, 477-9.

Yu, G., Wang, L. G., Han, Y. and He, Q. Y. (2012). clusterProfiler: an R package for comparing biological themes among gene clusters. OMICS 16, 284-7.

Zhang, Q., Lenardo, M. J. and Baltimore, D. (2017). 30 Years of NF-kappaB: A Blossoming of Relevance to Human Pathobiology. Cell 168, 37-57.

Zhu, X., Liu, J., Xu, X., Zhang, C. and Dai, D. (2015). Genome-wide analysis of histone modifications by ChIP-chip to identify silenced genes in gastric cancer. Oncol Rep 33, 2567-74. 
1054

1055

1056

1057

1058

1059

1060

1061

1062

1063

1064

1065

1066

1067

1068

1069

1070

1071

1072

1073

1074

1075

1076

1077

\section{Acknowledgments}

We thank A. Zeigerer (Helmholtz Zentrum Munchen), D. Zdżalik-Bielecka, J. Cendrowski, A. Poświata, and M. Kaczmarek for critical reading of the manuscript. We also thank D. ZdżalikBielecka (International Institute of Molecular and Cell Biology) for help with pilot FACS experiments. We are grateful to A. Paziewska and A. Dąbrowska (Maria Skłodowska-Curie National Research Institute of Oncology) for their technical support in RNA-Seq analysis.

\section{Competing interests}

The authors declare that they have no competing interests.

\section{Author contributions}

The research was conceived by M. Miączyńska, K.K. and M. Mikula. Funding was acquired by M. Miączyńska. Experiments were designed and performed mostly by K.K. P.E., B.S. and E.S., with input from M. Miączyńska, E.S. and crucial help from M. Mikula and K.G. (RNA-Seq), E.D-W, A.S-C. and M.P-S. (immunohistochemistry), and MB-O. and K.P. (flow cytometry). The manuscript was written by K.K. and M. Miączyńska. Figures were assembled by K.K. with help of A.S-C. K.K. and M. Miączyńska supervised the work. All authors approved the manuscript.

\section{Funding}

This study was financed by TEAM grant (POIR.04.04.00-00-20CE/16-00), K. Piwocka was supported by TEAM-TECH Core Facility Plus/2017-2/2 grant (POIR.04.04.00-00-23C2/17-00) - both grants from the Foundation for Polish Science co-financed by the European Union under the European Regional Development Fund. E. Szymańska was supported by Sonata grant (2016/21/D/NZ3/00637) from the National Science Center.

\section{Data and materials availability}

The RNA-Seq datasets have been deposited to GEO under the accession number: GSE152195 (https://www.ncbi.nlm.nih.gov/geo/query/acc.cgi?acc=GSE152195). 


\section{Fig. 1}
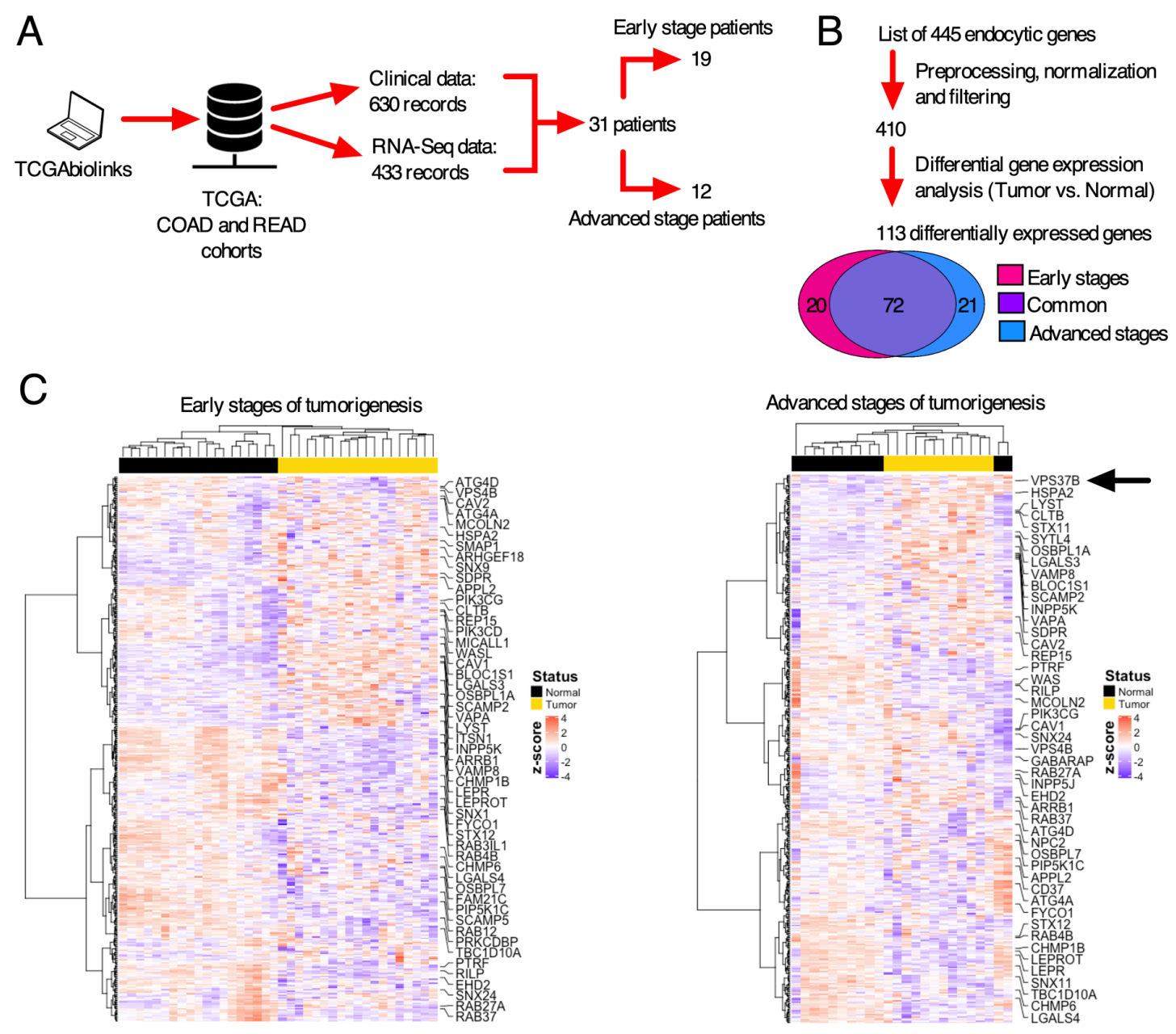

D
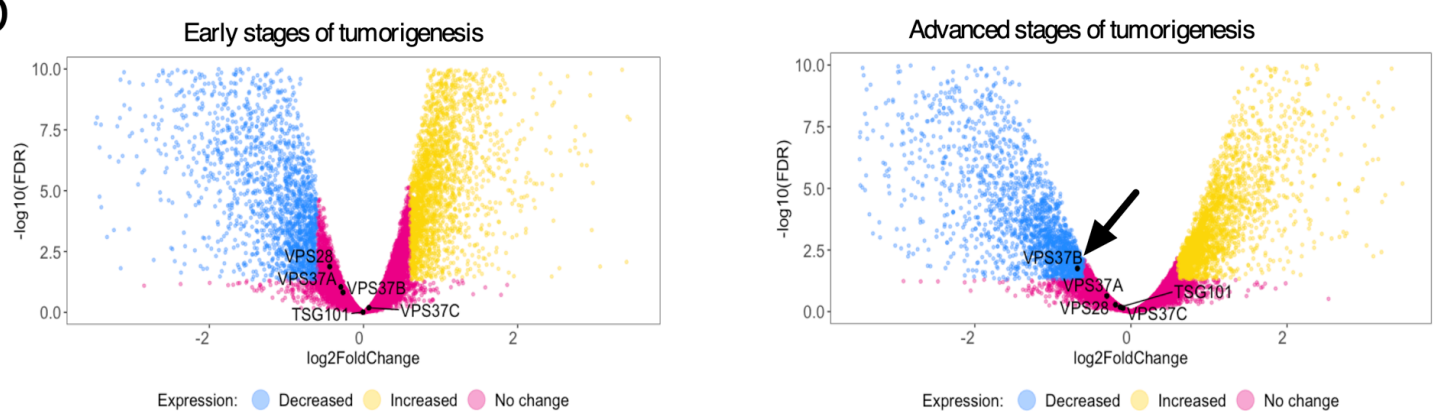

Fig. 1. Expression of $V P S 37 B$ is decreased in advanced stages of colorectal cancer. (A) Scheme of data mining process of The Cancer Genome Atlas (TCGA) database with a focus on

1081 the COAD and READ cohorts using the TCGAbiolinks package. (B) Scheme showing the 1082 number of genes in consecutive stages of analysis and Venn diagram of differentially expressed 
1083 genes related to endocytic transport in patients at the early (stage I and II) and advanced stages 1084 (stage III and IV) of CRC. (C) Heatmaps visualizing the expression of the genes with decreased 1085 expression in early stages and advanced stages of CRC. Columns are samples from normal tissue 1086 or tumor. Rows are transcripts. (D) Volcano plots visualizing the expression of ESCRT genes in 1087 early and advanced stages of CRC. Genes with increased and decreased expression are those 1088 with False Discovery Rate $($ FDR $)<0.05$ and log2FoldChange $\geq 0.6$ and $\leq-0.6$, respectively. 


\section{Fig. 2}

A

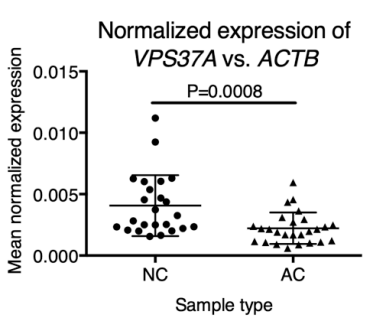

C

Vps37A staining intensity

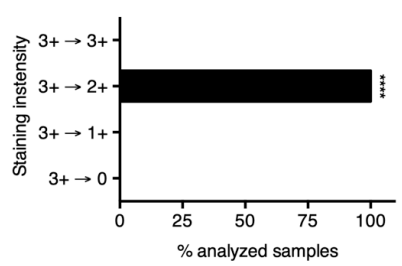

1089

1090

1091

1092

1093

1094

1095

1096

1097

1098

1099

Fig. 2. Abundance of $V P S 37 A$ and $V P S 37 B$ paralogs is decreased in a treatment-naïve cohort of CRC patients. (A) qRT-PCR analysis of VPS37A and VPS37B mRNA levels in normal colon $(\mathrm{NC}, \mathrm{n}=24)$ and adenocarcinoma $(\mathrm{AC}, \mathrm{n}=26)$ samples. Middle lines are means and whiskers are standard deviation. Differences were analyzed using the Mann-Whitney U test. (B) Examples of IHC staining of Vps37A and Vps37B in normal colon and matched CRC samples as an illustration of the scoring system used for the evaluation presented in (C). Scale bars: 200 $\mu \mathrm{m}$. (C) Comparative analysis of Vps37A and Vps37B IHC staining performed in pairs of normal colon and matched CRC samples $(n=100)$ : $3+-$ very intense staining, $2+-$ medium intense staining, $1+-$ weak staining, $0-$ no staining. Statistical significance was assessed against healthy tissue using Fisher's exact test $* * * * P<0.0001$. 
bioRxiv preprint doi: https://doi.org/10.1101/2020.07.02.183954; this version posted July 3, 2020. The copyright holder for this preprint (which was not certified by peer review) is the author/funder. All rights reserved. No reuse allowed without permission.

Fig. 3

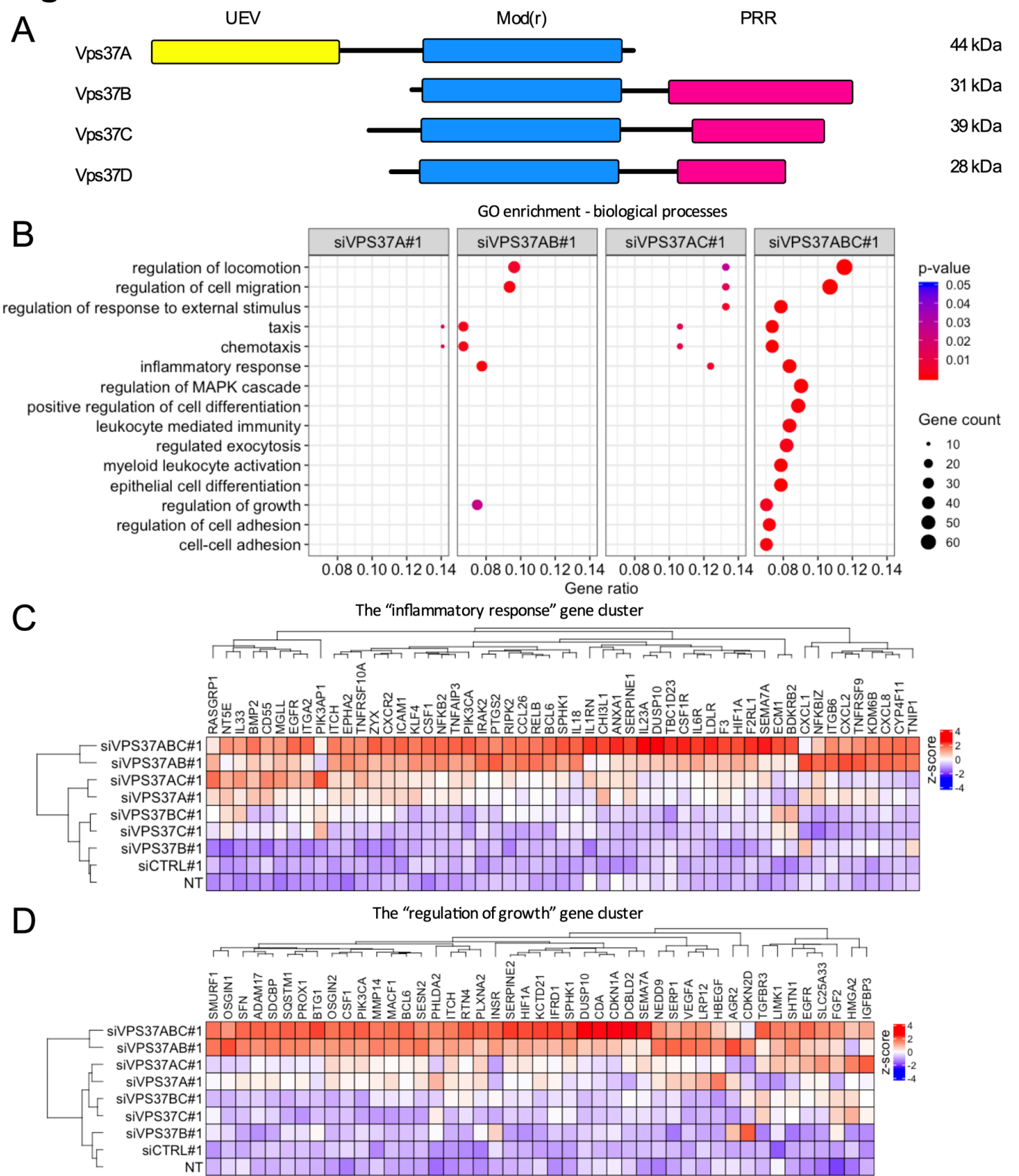

E

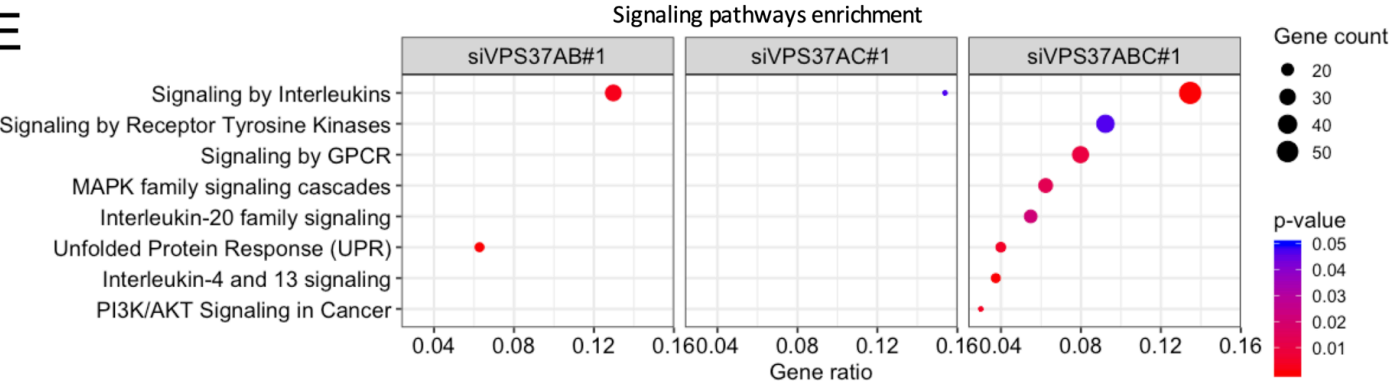

1100 


\section{Fig. 3. Concurrent depletion of Vps37 proteins induces multiple transcriptional responses}

1102 in DLD1 cells. (A) Domain architecture of human VPS37 paralogs. UEV - ubiquitin enzyme 1103 variant, Mod(r) - modifier of rudimentary, PRR - proline rich region. (B) Top 15 biological

1104 processes from gene ontology (GO) analysis among differentially expressed genes $(\geq 1.50$-fold or $1105 \leq 0.667$-fold; adjusted $P<0.05$ ) after individual or concurrent silencing of VPS37 paralogs. 1106 Analysis was performed using the enrichGO function from clusterProfiler. (C-D) Heatmaps

1107 visualizing expression of genes related to inflammatory response (C) and regulation of cell 1108 growth (D) generated from the GO analysis of biological processes across different transfection 1109 conditions. (E) Selected pathways from the signaling pathway analysis among differentially 1110 expressed genes after individual or concurrent silencing of VPS37 paralogs. Analysis was 1111 performed using the enrichPathway function from ReactomePA. RNA-Seq data analysis was 1112 performed using $\mathrm{n}=3$ independent experiments. NT - non-transfected cells, abbreviations for 1113 differentially transfected cells are explained in Materials and Methods. 
Fig. 4
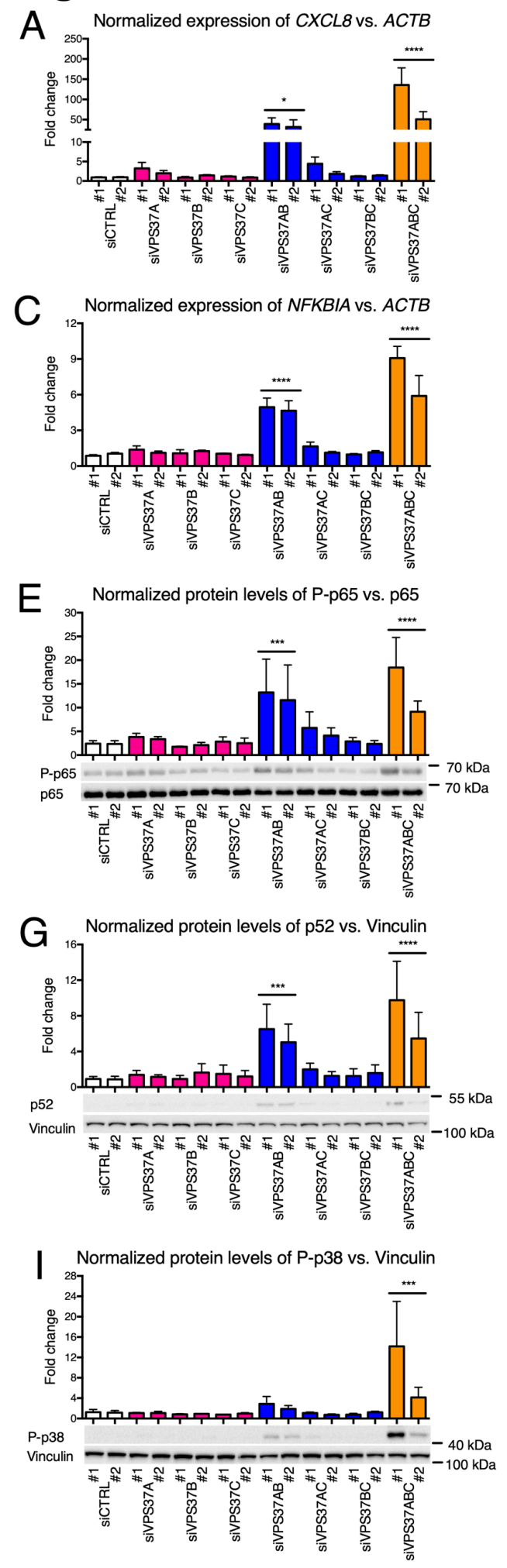

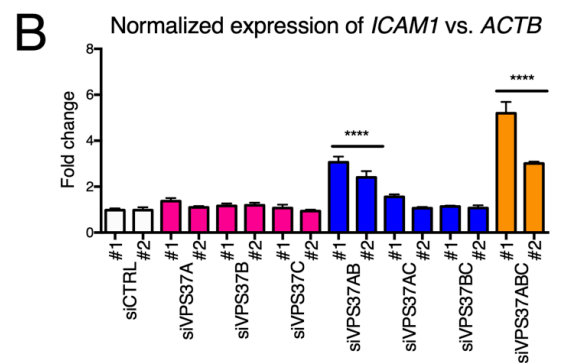

Normalized expression of TNFAIP3 vs. ACTB

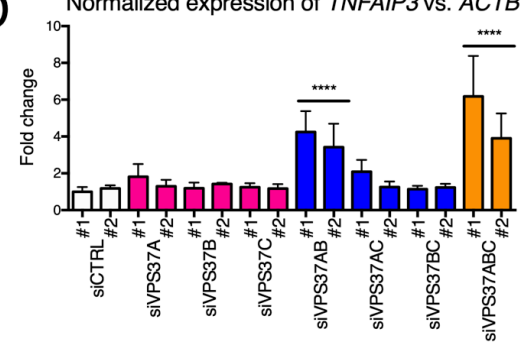

F Normalized protein levels of $\mathrm{p} 100 \mathrm{vs.}$. Vinculin
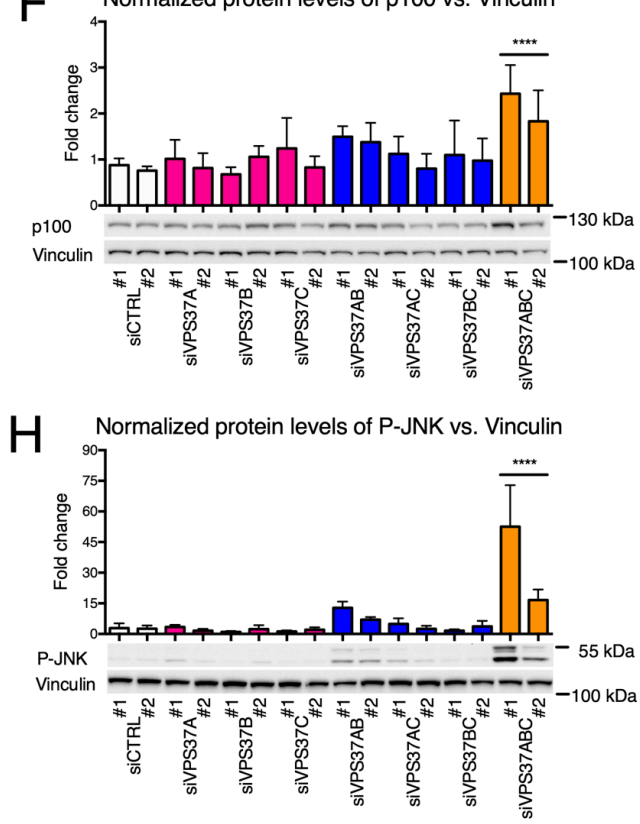

Normalized protein levels of P-ERK vs. Vinculin

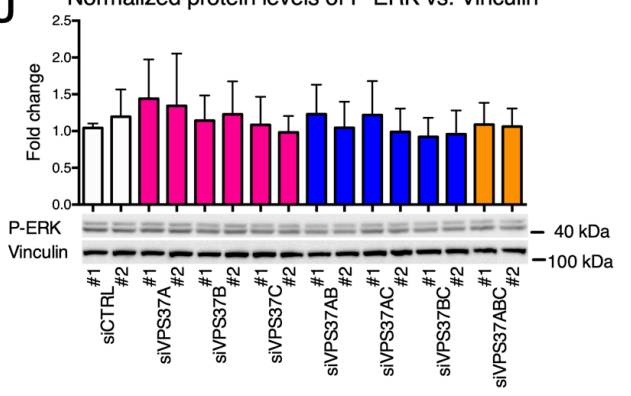

1114 
1115 Fig. 4. Concurrent depletion of Vps37 proteins induces MAPK and NF-кB inflammatory

1116 responses in RKO cells. (A-D) qRT-PCR analysis of expression of selected genes in the

1117 inflammatory response cluster: (A) CXCL8, (B) ICAM1, (C) NFKBIA and (D) TNFAIP3,

1118 measured $72 \mathrm{~h}$ after transfection with siRNA targeting VPS37 paralogs individually or in

1119 combinations. Non-transfected (NT) cells and transfected with non-targeting siRNA (siCTRL)

1120 were used to assess the basal expression level of the investigated genes. ACTB (encoding $\beta$ -

1121 Actin) was used as a reference gene. (E-G) Western blotting analysis of the NF- $\kappa$ B pathway

1122 activation: (E) phosphorylation of p65, (F) p100 and (G) p52 protein abundance. (H-J) Western

1123 blotting analysis of MAPK activation: (H) phosphorylation of JNK, (I) phosphorylation of p38

1124 and (J) phosphorylation of ERK. (E-J) Lysates of RKO cells were collected $72 \mathrm{~h}$ after

1125 transfection with siRNA targeting VPS37 paralogs individually or in combinations. Lysates from

1126 non-transfected (NT) cells and transfected with non-targeting siRNA (siCTRL) were used to

1127 assess the basal level of intracellular signaling. p65 and vinculin were used as loading controls.

1128 Representative blots are shown along with densitometry analysis. Data in all panels are mean \pm 1129 standard deviation of $\mathrm{n}=3$ independent experiments expressed as the fold change of either mRNA 1130 (A-D) or protein (E-J) levels in NT cells, which was set as 1. Statistical significance was 1131 assessed against grouped siCTRL conditions using one-way ANOVA test followed by 1132 Bonferroni's correction; $* P<0.05$, ** $P<0.01$, *** $P<0.001$, **** $P<0.0001$. 
Fig. 5

A

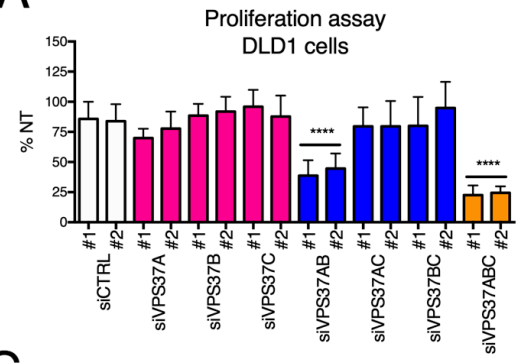

C

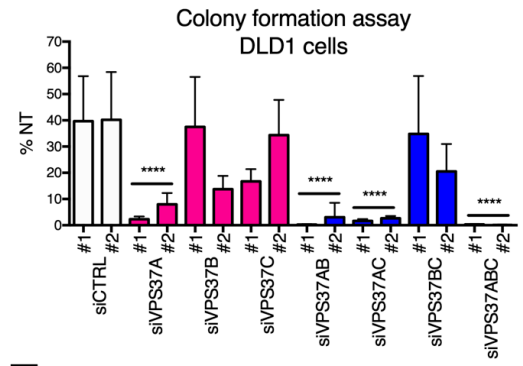

$\mathrm{E}$

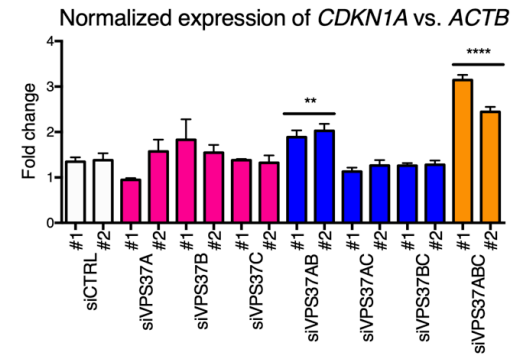

G

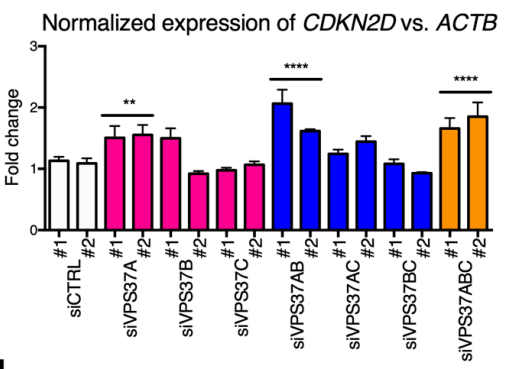

B

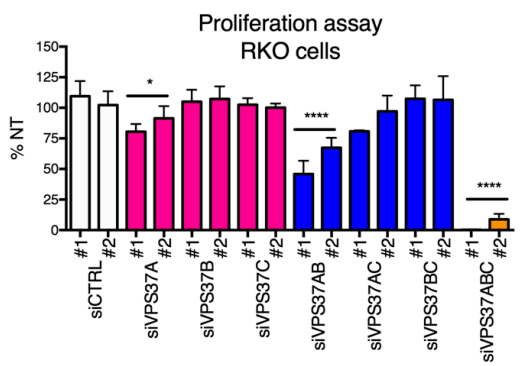

$\mathrm{D}$

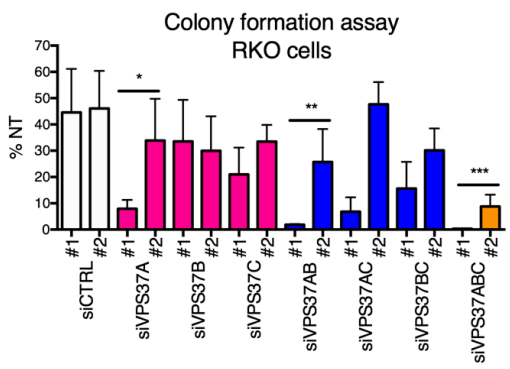

$\mathrm{F}$

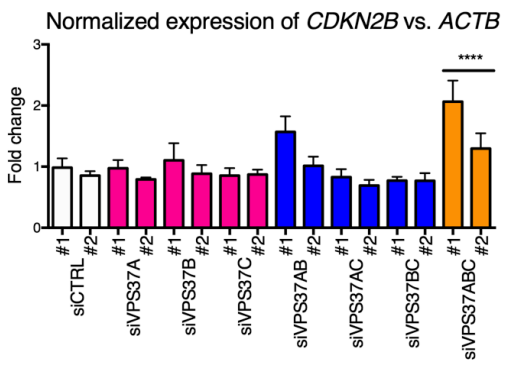

$\mathrm{H}$

Proliferation assay

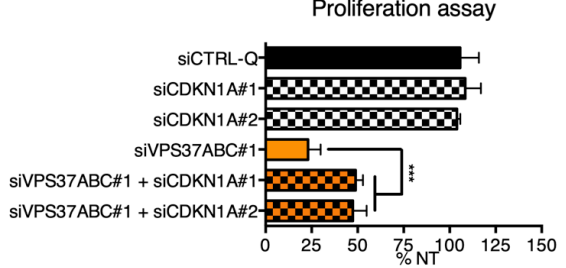

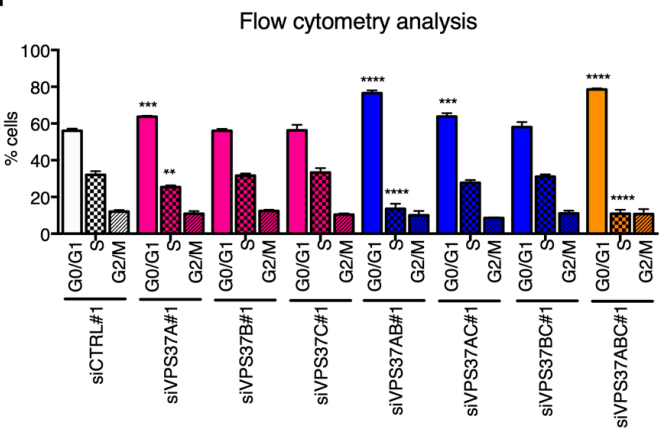


1134 Fig. 5. Concurrent depletion of Vps37 proteins inhibits CRC cell growth in vitro. (A, B) Cell 1135 proliferation of (A) DLD1 and (B) RKO cells assessed $120 \mathrm{~h}$ after individual and concurrent 1136 depletion of VPS37 paralogs using the BrdU proliferation assay. (C, D) Clonogenic growth of

1137 (C) DLD1 and (D) RKO cells assessed 14 days after individual and concurrent knockdown of 1138 VPS37 paralogs with representative images for each transfection condition shown in Fig. S4A for 1139 DLD1 cells and Fig. S4B for RKO cells. (E-G) qRT-PCR analysis of expression of genes 1140 encoding CDKNs: (E) CDKN1A, (F) CDKN2B and (G) CDKN2D, measured $72 \mathrm{~h}$ after individual 1141 and concurrent depletion of Vps37 paralogs. Non-transfected (NT) cells and transfected with 1142 non-targeting siRNA were used to assess the basal level of the investigated genes. $A C T B$ 1143 (encoding $\beta$-Actin) was used as a reference gene. $(\mathrm{H})$ Cell proliferation of RKO cells was 1144 assessed $120 \mathrm{~h}$ after concurrent silencing of all VPS37 paralogs and CDKN1A using the 1145 proliferation assay. (I) Analysis of cell cycle was performed upon individual or concurrent 1146 silencing of VPS37 paralogs. Cells were forward transfected for $96 \mathrm{~h}$, stained with PI and 1147 evaluated with a flow cytometer. Data presented in all panels are mean of $n=3$ independent 1148 experiments \pm standard deviation analyzed with one-way ANOVA with Bonferroni's correction. 1149 Statistical significance for grouped siCTRL conditions (A-G, I) and for siCTRL\#1-Q (I); $1150 * P<0.05 ; * * P<0.01, * * * P<0.001, * * * * P<0.0001$. 
Fig. 6

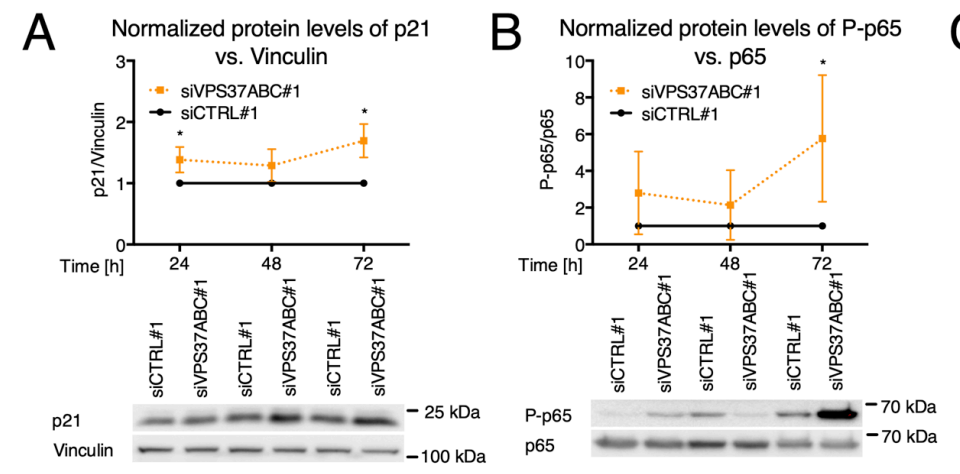

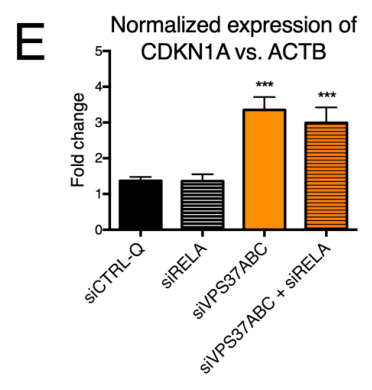

D

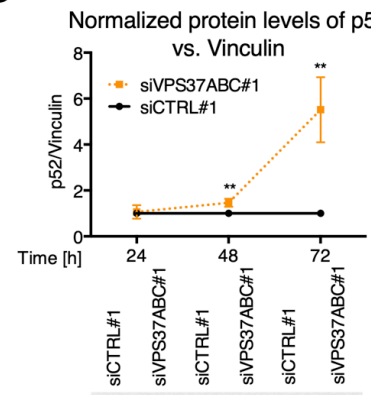

$\mathrm{p} 52$
Vinculin $--\square-\square-55 \mathrm{kDa}$
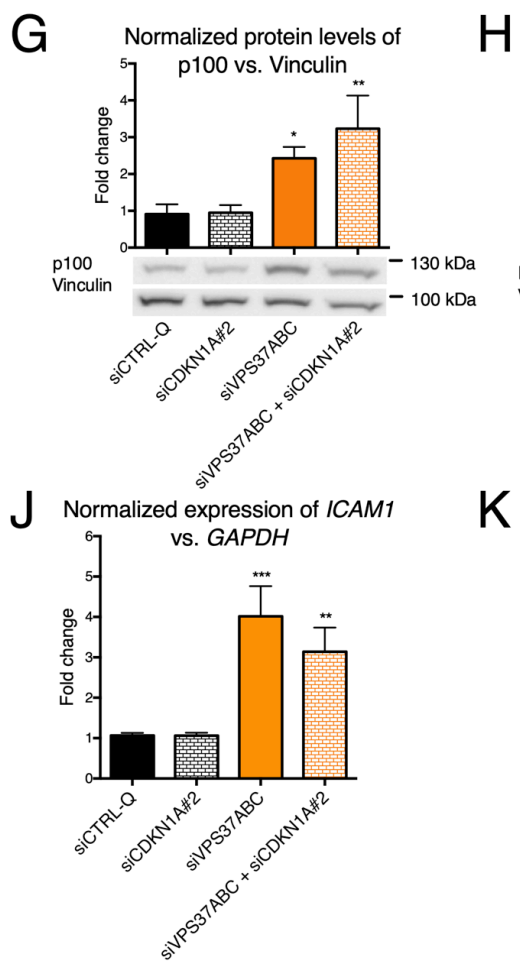

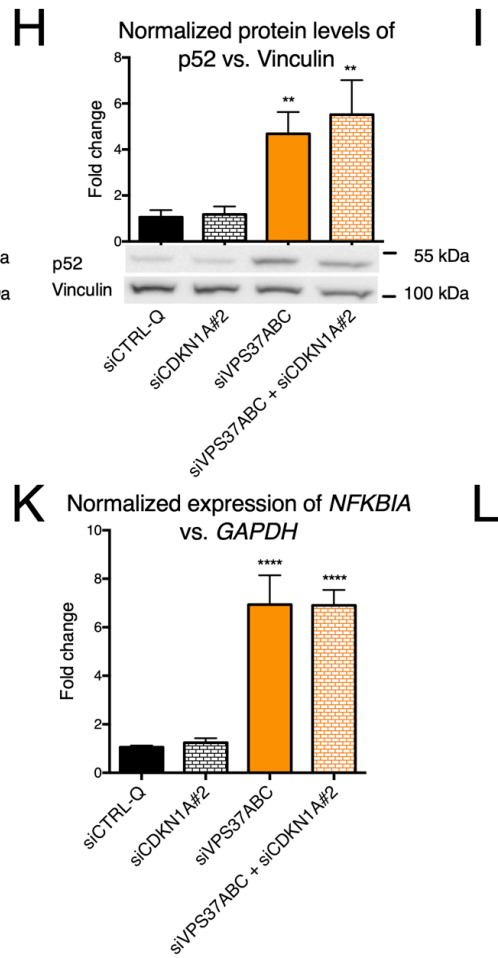

1151
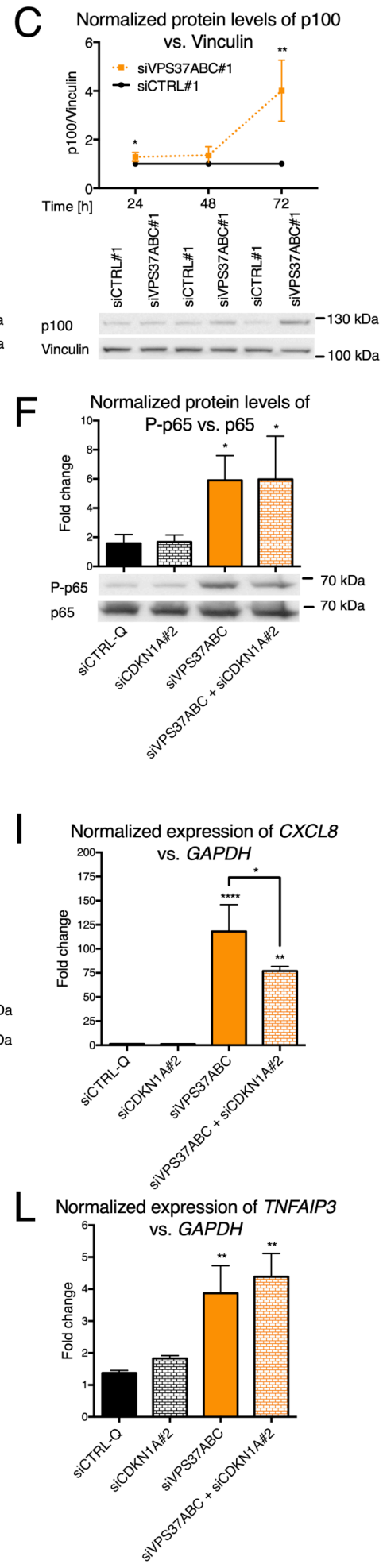
1152 Fig. 6. Inflammatory response and cell growth inhibition after concurrent depletion of 1153 VPS37 paralogs are two independently regulated processes in RKO cells. (A-D) Western 1154 blot analyses of (A) p21, (B) phosphorylated p65, (C) p100 and (D) p52 abundance were 1155 performed 24, 48 and $72 \mathrm{~h}$ after transfection with non-targeting or on-target siRNA for $1156 V P S 37 A B C$. (E) qRT-PCR analysis of CDKN1A was performed $72 \mathrm{~h}$ after transfection with 1157 siRNA targeting the p65 subunit of NF- $\mathrm{kB}$ dimer (RELA) alone or in combination with three 1158 VPS37 paralogs. (F-H) Western blotting analyses of (F) phosphorylated p65, (G) p100 and (H) $1159 \mathrm{p} 52$ abundance were performed $72 \mathrm{~h}$ after transfection with siRNA targeting CDKN1A alone or 1160 in combination with all the three VPS37 paralogs. (I-L) qRT-PCR analyses of (I) CXCL8, (J) 1161 ICAM1, (K) NFKBIA, and (L) TNFAIP3 were performed $72 \mathrm{~h}$ after transfection with siRNA 1162 targeting $C D K N 1 A$ alone or in combination with all the three VPS37 paralogs. (E, I-L) ACTB 1163 (encoding $\beta$-Actin) or GAPDH (encoding Glyceraldehyde 3-phosphate dehydrogenase) were 1164 used as a reference gene in qRT-PCR analysis. (A-D, F-H) p65 and vinculin were used as 1165 loading controls for Western blotting. Representative blots are shown along with densitometry 1166 analysis. Data in all panels are mean \pm standard deviation of $n=3$ independent experiments 1167 expressed as the fold change of either mRNA or protein level. In panels A-D protein abundance 1168 in siCTRL\#1 transfected cells was set to 1, whilst in panels E-L mRNA and protein abundance in 1169 non-transfected (NT) cells was set as 1. Statistical significance was assessed using unpaired 1170 Student's t-test (A-D) or one-way ANOVA test followed by Bonferroni's correction (E-L).

1171 Statistical significance against siCTRL\#1 at matching time point for transfection with three 1172 different siRNA (A-D) and siCTRL-Q for four different siRNA (E-L), $* P<0.05, * * P<0.01$, $1173 * * * P<0.001, * * * * P<0.0001$. 


\section{Fig. 7}
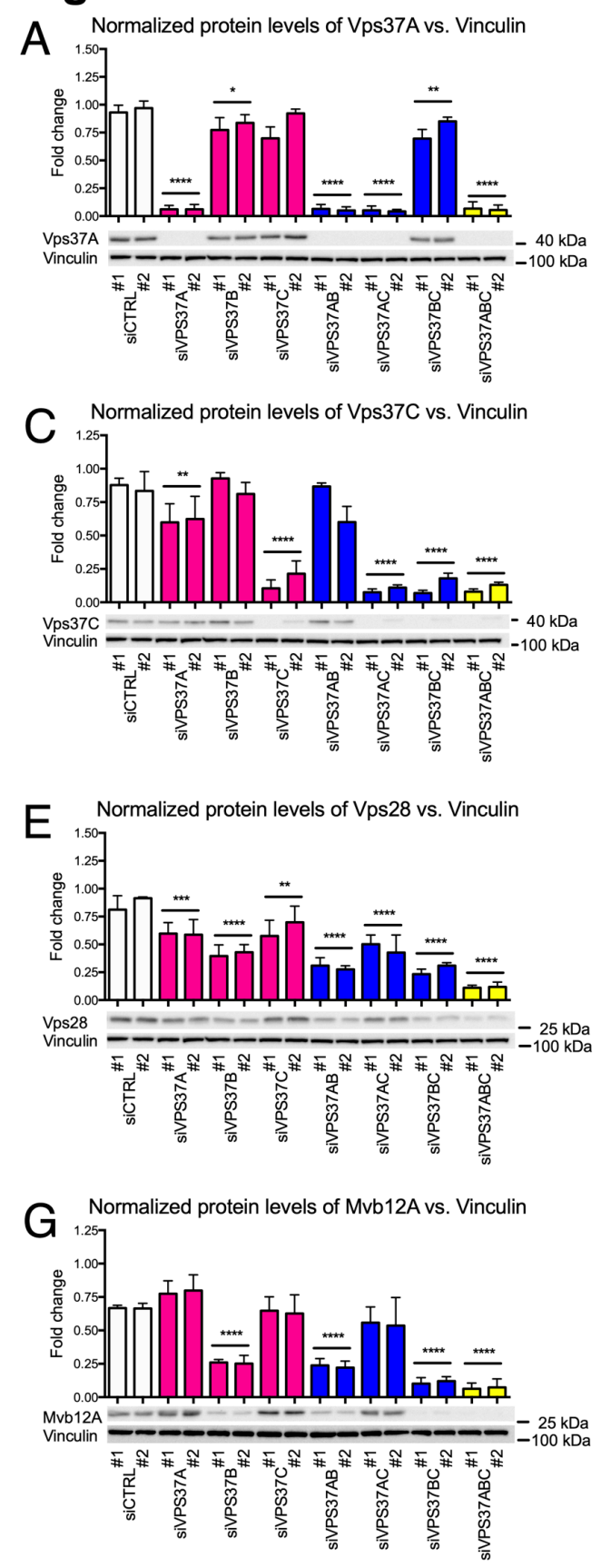
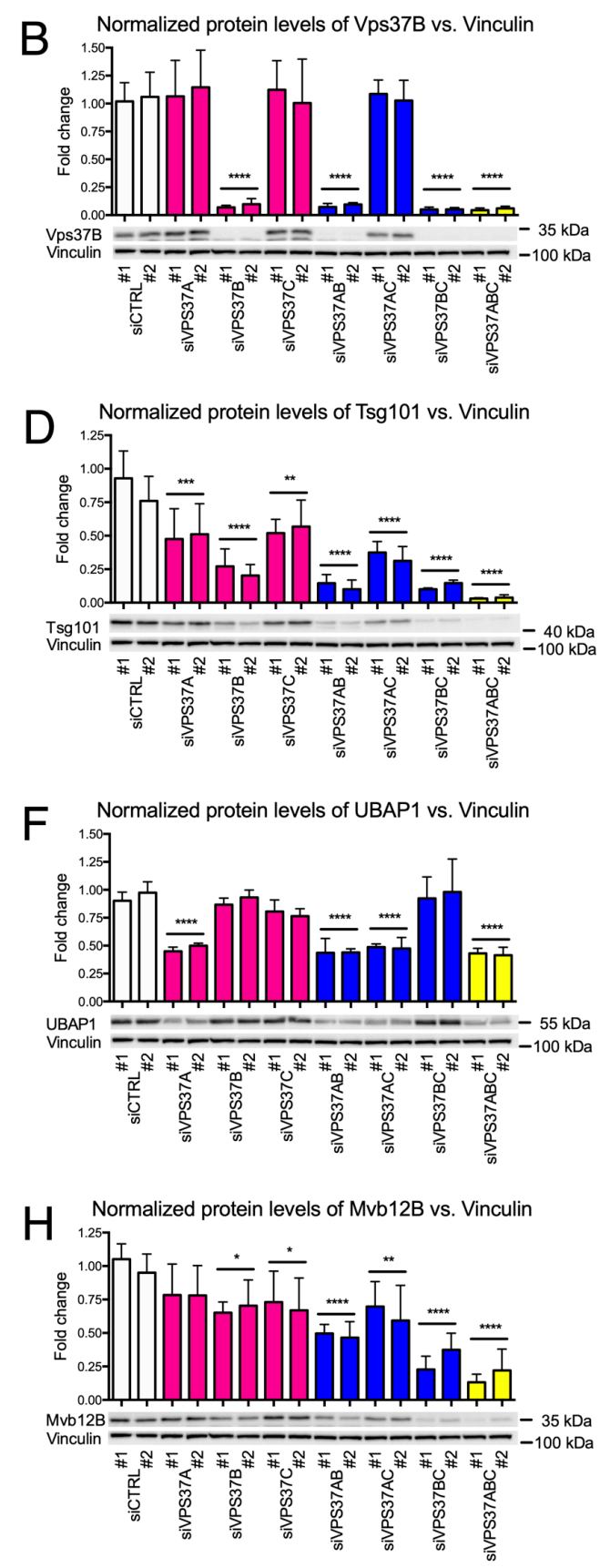

Fig. 7. Concurrent depletion of Vps37 proteins destabilizes ESCRT-I components and reveals partnering preferences for auxiliary subunits. Western blotting analysis of ESCRT-I subunits: (A) Vps37A, (B) Vps37B, (C) Vps37C, (D) Tsg101, (E) Vps28, (F) UBAP-1, (G) Mvb12A and (H) Mvb12B. Lysates of RKO cells were collected $72 \mathrm{~h}$ after transfection with siRNA targeting VPS37 paralogs individually or in combinations. Lysates of RKO cells from 
1180 non-transfected (NT) cells and transfected with non-targeting siRNA were used to assess the 1181 basal level of ESCRT-I subunits. Vinculin was used as a loading control. Representative blots 1182 are shown along with densitometry analysis. Data are mean \pm standard deviation of $n=3$ 1183 independent experiments expressed as the fold change of protein level in NT cells, which was set 1184 as 1. Statistical significance was assessed using ANOVA test followed by Bonferroni's 1185 correction. Statistical significance against siCTRL conditions, ${ }^{*} P<0.05, * * P<0.01, * * * P<0.001$, $1186 \quad * * * * P<0.0001$. 

response cluster. Transcriptional motif-enrichment analysis of the inflammatory response gene cluster identified in our RNA-Seq analysis was performed using RcisTarget. A region of $500 \mathrm{bp}$ upstream and $100 \mathrm{bp}$ downstream to the transcription starting site was investigated with the TFBS matrices from the JASPAR database.

\begin{tabular}{|c|c|c|c|}
\hline $\begin{array}{l}\text { Transcription } \\
\text { Factor }\end{array}$ & $\begin{array}{l}\text { Consensus } \\
\text { sequence }\end{array}$ & $\begin{array}{l}\text { Normalized } \\
\text { Enrichment } \\
\text { Score }\end{array}$ & $\begin{array}{l}\text { Genes from the inflammatory } \\
\text { response cluster }\end{array}$ \\
\hline $\begin{array}{l}\text { RELA } \\
(\mathrm{p} 65)\end{array}$ & & 7.72 & $\begin{array}{l}\text { BCL6, CSF1, CXCL1, CXCL2, } \\
\text { CXCL8, F3, NKFB2, NFKBIZ, } \\
\text { PTGS2, RELB, RIPK2, TNFAIP3, } \\
\text { TNIP, ZYS }\end{array}$ \\
\hline $\begin{array}{l}\text { REL } \\
(\mathrm{p} 65 / \text { RelB/c- } \\
\text { Rel) }\end{array}$ & & 6.21 & $\begin{array}{l}\text { BCL6, CSF1, CXCL1, CXCL2, } \\
\text { CXCL8, IL18, IL1RN, LDLR, } \\
\text { NFKB2, NFKBIZ, RELB, RIPK2, } \\
\text { SEMA7A, TNFAIP3, TNIP, ZYS }\end{array}$ \\
\hline FOSL1 (Fra-1) & & 3.38 & $\begin{array}{l}\text { BCL6, CXCL8, ECM1, F3, IL1RN, } \\
\text { IL23A, SPHK1, TNFAIP3 }\end{array}$ \\
\hline JUNB (Jun-B) & & 3.29 & $\begin{array}{l}\text { BCL6, CXCL8, ECM1, F2, IL1RN, } \\
\text { IL23A, SNHK1, TNFAIP3 }\end{array}$ \\
\hline JUN (c-Jun) & & 3.25 & $\begin{array}{l}\text { BCL6, CXCL8, ECM1, F3, IL1RN, } \\
\text { IL23A, SPHK1, TNFAIP3 }\end{array}$ \\
\hline FOSL2 (Fra-2) & & 3.17 & $\begin{array}{l}\text { BCL6, CXCL8, ECM1, F3, IL1RN, } \\
\text { IL23A, SPHK1, TNFAIP3 }\end{array}$ \\
\hline JUND (Jun-D) & & 3.02 & $\begin{array}{l}\text { BCL6, CXCL8, ECM1, F3, IL1RN, } \\
\text { IL23A, SPHK1, TNFAIP3 }\end{array}$ \\
\hline
\end{tabular}




\section{Fig. S1}

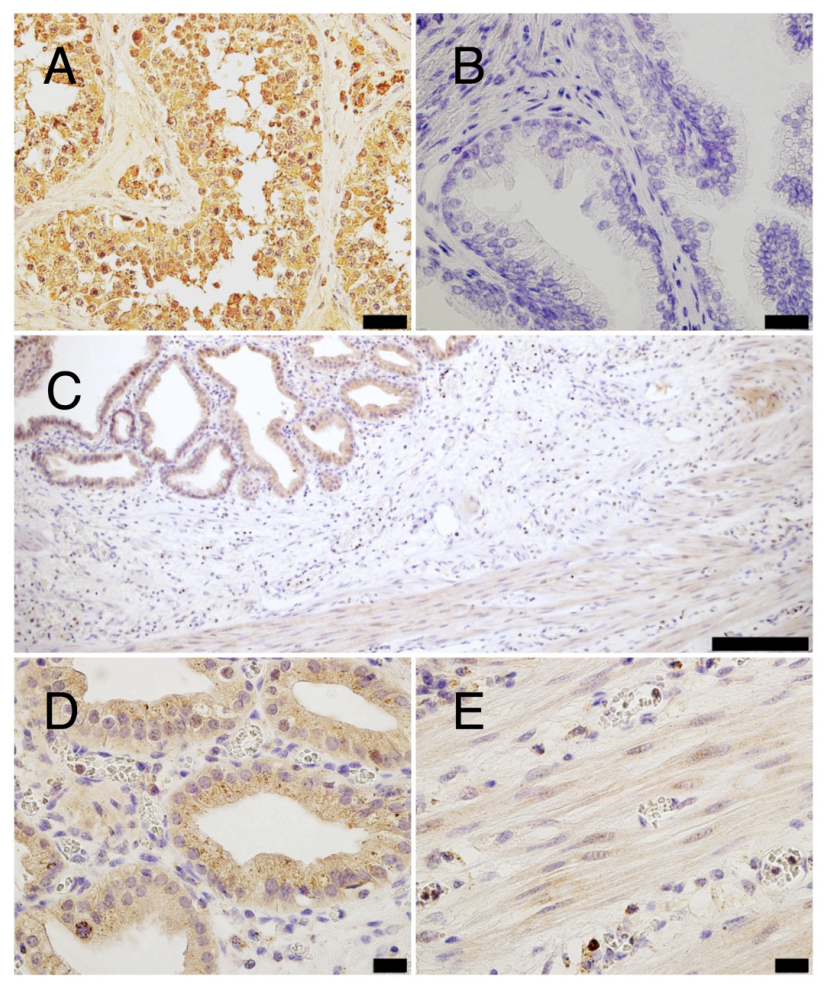

1194 Fig. S1. Immunohistochemical evaluation of the specificity of antibodies against Vps37A

1195 and Vps37B proteins. Vps37A: (A) positive control - strong granular cytoplasmic staining in

1196 the glandular epithelium of testis (scale bar $20 \mu \mathrm{m}$ ), (B) lack of staining in the glandular 1197 epithelium of prostate (scale bar $10 \mu \mathrm{m}$ ). Vps37B: (C) positive control - strong granular 1198 cytoplasmic staining in the mucosa of the gallbladder (scale bar $100 \mu \mathrm{m}$ ) and (D) at higher 1199 magnification (scale bar $10 \mu \mathrm{m}$ ), (E) weak staining in the muscle (scale bar $10 \mu \mathrm{m}$ ). 


\section{Fig. S2}
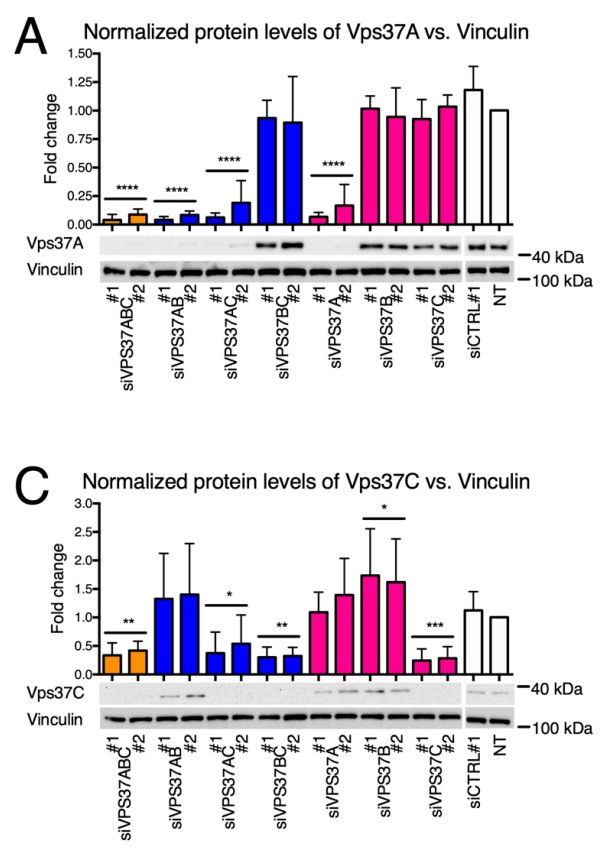

B

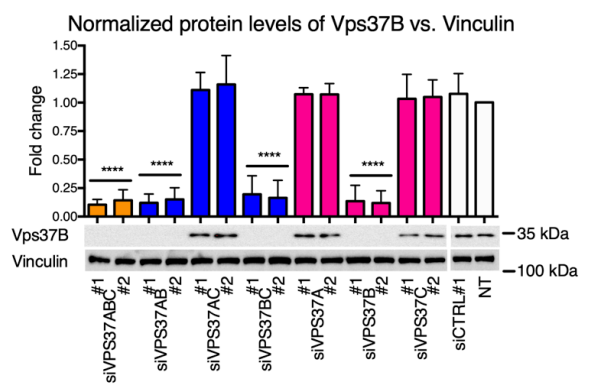

D

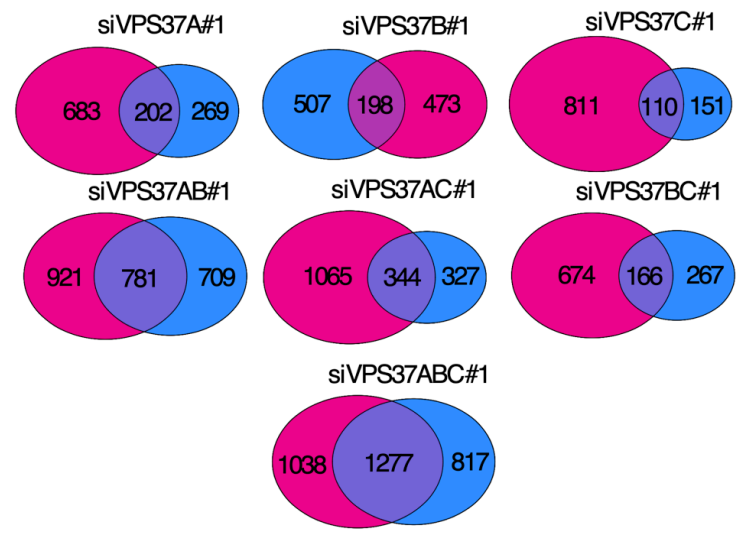

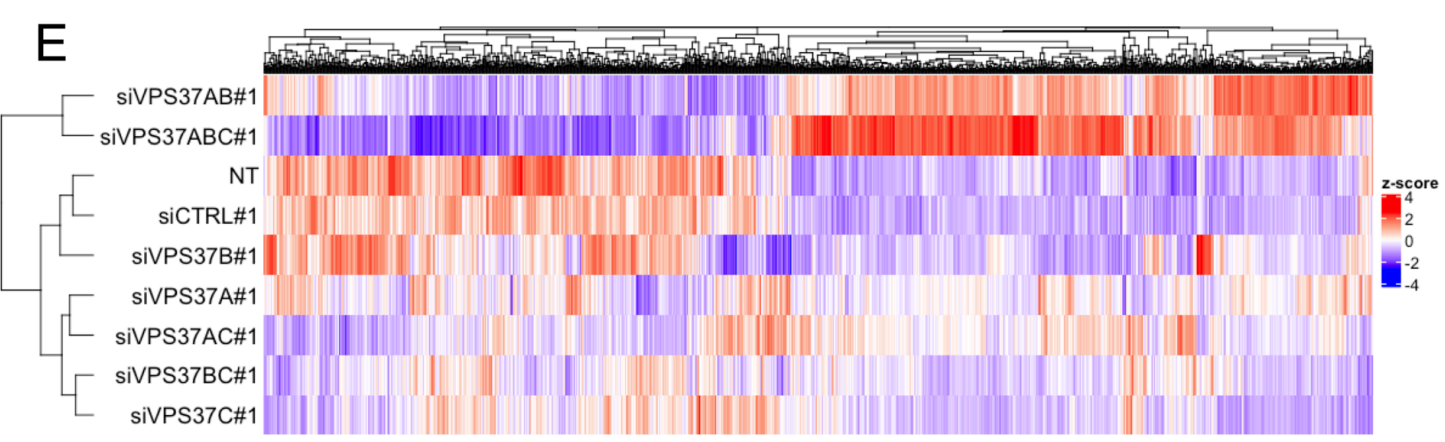
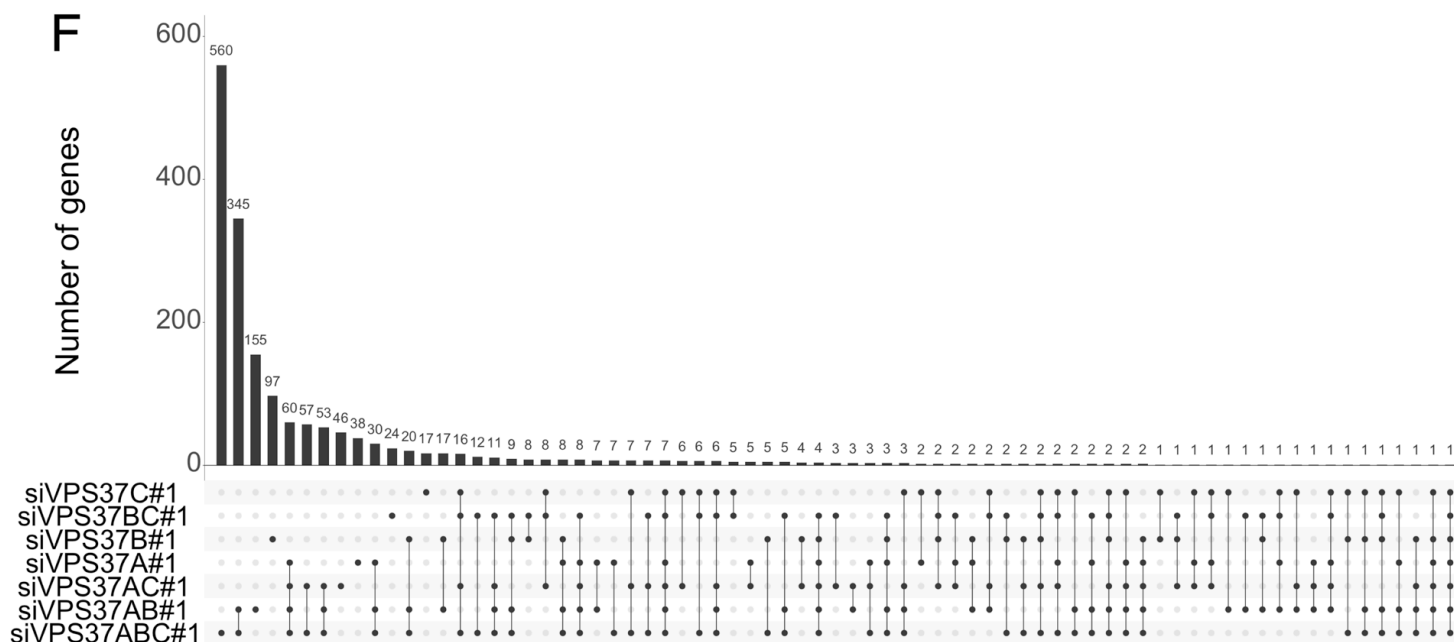
1201 Fig. S2. Transcriptional alteration after individual or combined knockdown of VPS37 1202 paralogs in DLD1 cells. (A-C) Western blotting analysis of selectivity of siRNAs for the VPS37 1203 paralogs: (A) VPS37A, (B) VPS37B and (C) VPS37C. Lysates were collected $72 \mathrm{~h}$ after 1204 transfection with siRNA targeting individual or combinations of VPS37 paralogs. Lysates from 1205 non-transfected (NT) cells and transfected with non-targeting siRNA (siCTRL\#1) were used to 1206 assess the basal level of intracellular signaling. Vinculin was used as a loading control. 1207 Representative blots from $\mathrm{n}=5$ independent experiments are shown along with densitometry 1208 analysis. Data are mean \pm standard deviation expressed as the fold change of protein level in NT 1209 cells, which was set as 1. Statistical significance was assessed against grouped NT and 1210 siCTRL\#1 conditions using one-way ANOVA test followed by Bonferroni's correction; $1211 * P<0.05, * * P<0.01, * * * P<0.001, * * * * P<0.0001$. (D) Venn diagrams of differentially expressed 1212 genes $(\geq 1.50$-fold or $\leq 0.667$-fold; adjusted $\mathrm{P}<0.05)$ after single, double or triple VPS37 paralog 1213 silencing when normalized to non-transfected (NT, pink circles) cells and cells transfected with 1214 non-targeting siRNA (siCTRL\#1, blue circles). Differentially expressed genes were identified 1215 using DESeq2. (E) Heatmap visualizing clustering of expression patterns for differentially 1216 expressed genes in DLD1 cells across different transfection conditions. (F) Clustered heatmap 1217 showing pairwise intersections for the common pools of differentially expressed genes $(\geq 1.50$ 1218 fold or $\leq 0.667$-fold; adjusted $P<0.05$ ) after single, double or triple VPS37 paralog silencing 1219 when normalized to non-transfected cells and cells transfected with non-targeting siRNA for the 1220 investigated transfection conditions. RNA-Seq data analysis was performed using $\mathrm{n}=3$ 1221 independent experiments. 
Fig. S3

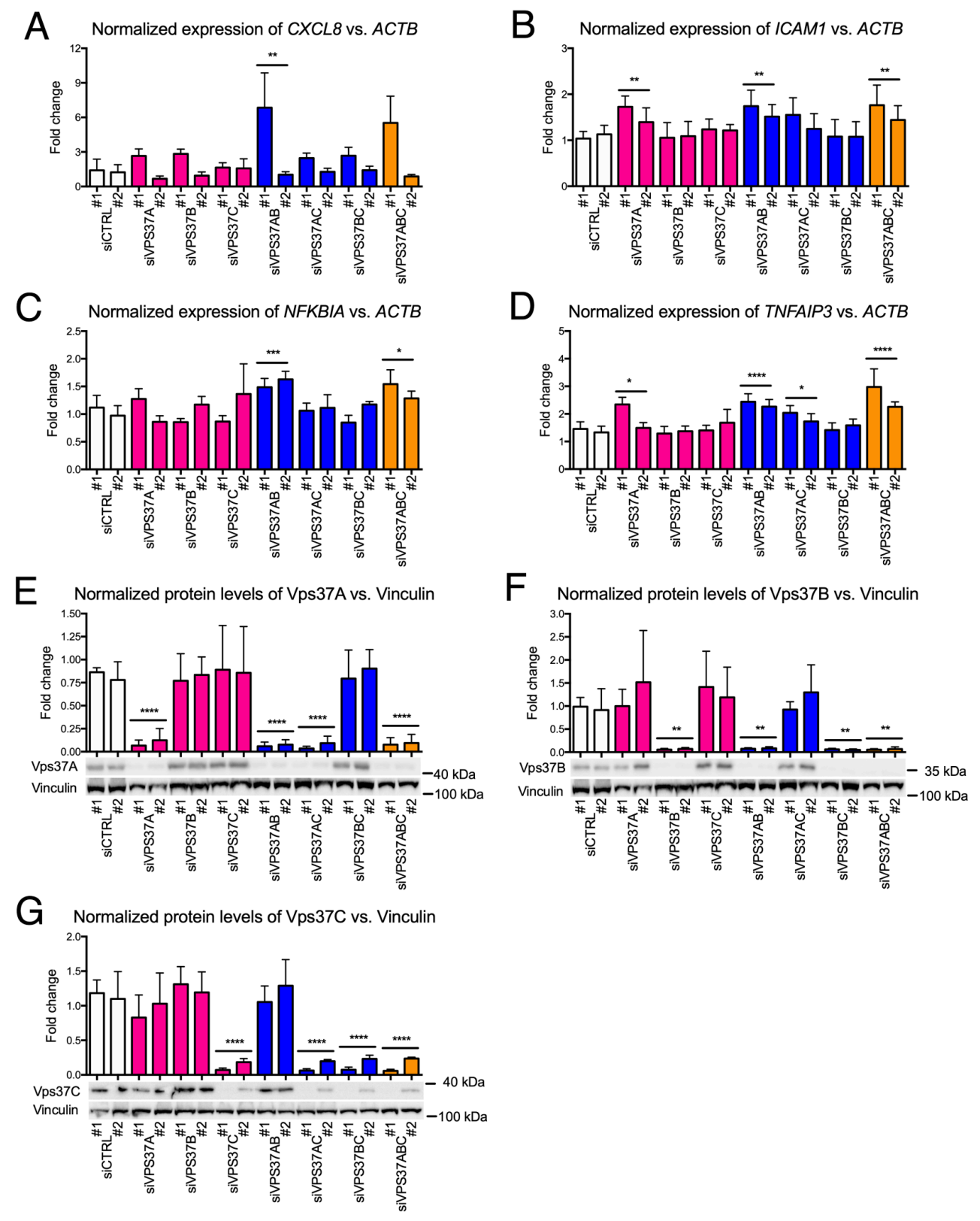

Fig. S3. Induction of inflammatory gene expression upon concurrent silencing of VPS37 paralogs. (A-D) qRT-PCR analysis of selected genes in the inflammatory response cluster: (A) CXCL8, (B) ICAM1, (C) NFKBIA and (D) TNFAIP3 in DLD1 cells. Expression of genes was measured $72 \mathrm{~h}$ after forward transfection with siRNA targeting of VPS37 paralogs individually or in combinations. Non-transfected (NT) cells and transfected with non-targeting siRNA (siCTRL) were used to assess the basal expression level of the investigated genes. ACTB 
1229 (encoding $\beta$-Actin) was used as a reference gene. Data in panels (A-D) are mean \pm standard 1230 deviation of $n=5$ independent experiments. Data are expressed as the fold change of mRNA

1231 levels in NT cells, which was set as 1. (E-G) Western blotting analysis of selectivity of siRNAs

1232 for the VPS37 paralogs. Lysates were collected $72 \mathrm{~h}$ after forward transfection with siRNA 1233 targeting individual or combinations of VPS37 paralogs. Lysates from NT cells and transfected 1234 with non-targeting siRNA (siCTRL\#1 and siCTRL\#2) were used to assess the basal level of 1235 intracellular signaling. Vinculin was used as a loading control. Representative blots from $n=3$

1236 independent experiments are shown along with densitometry analysis. Data are mean \pm standard 1237 deviation expressed as the fold change of protein level in NT cells, which was set as 1. Statistical 1238 significance in all panels was assessed against grouped siCTRL conditions using one-way 1239 ANOVA test followed by Bonferroni's correction; $\quad * P<0.05, \quad * * P<0.01, \quad * * * P<0.001$, $1240 \quad * * * * P<0.0001$. 
Fig. $\mathbf{S 4}$

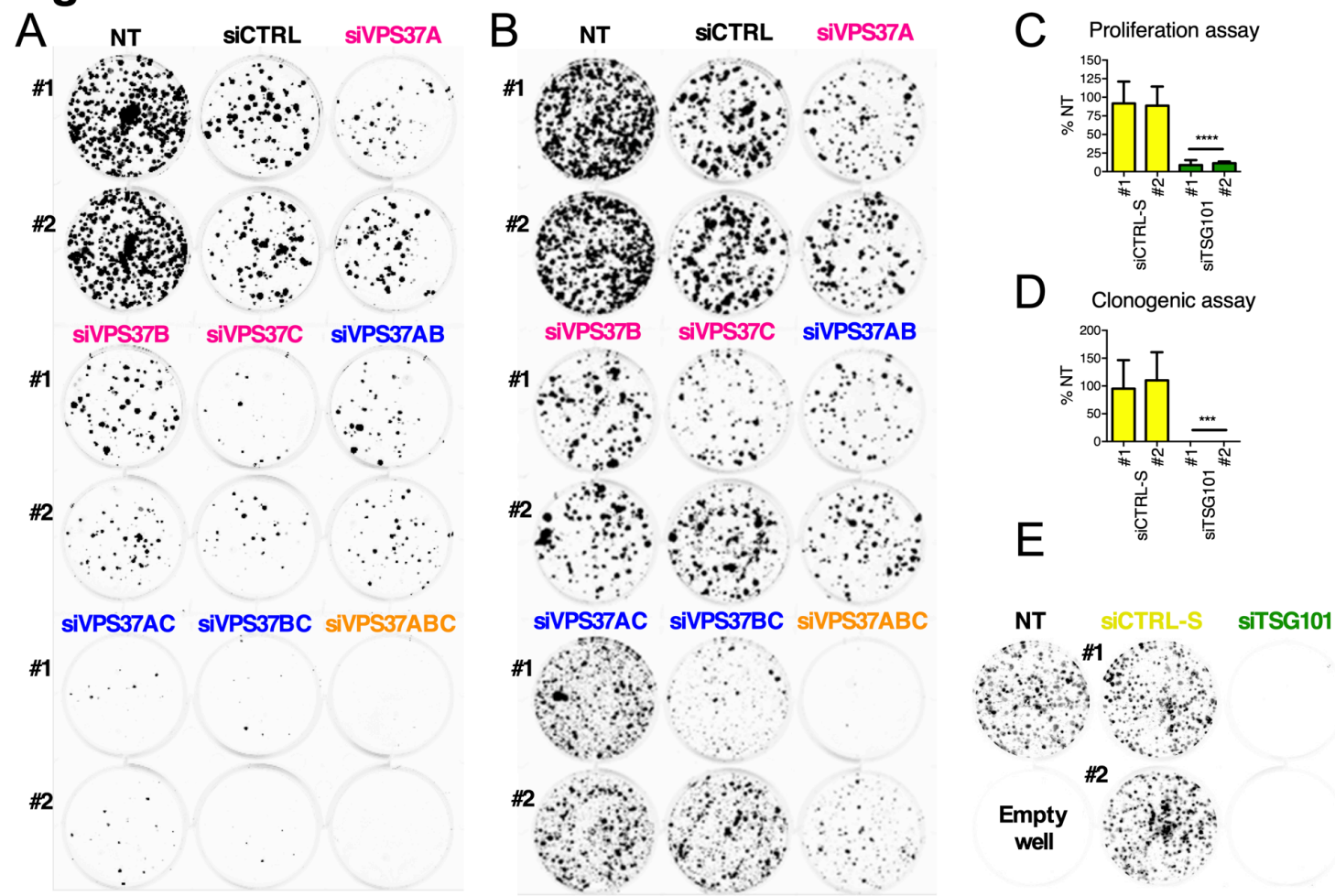

F Normalized expression of CDKN1A vs. ACTB

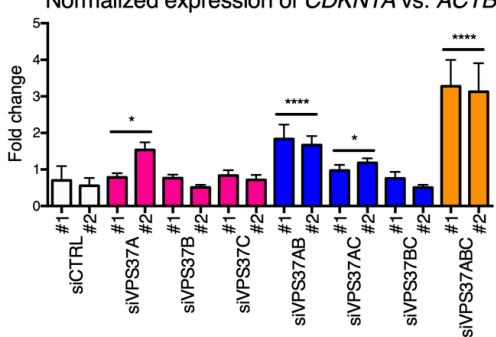

$\mathrm{H}$

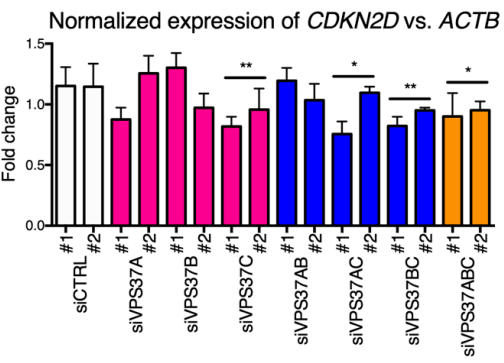

J

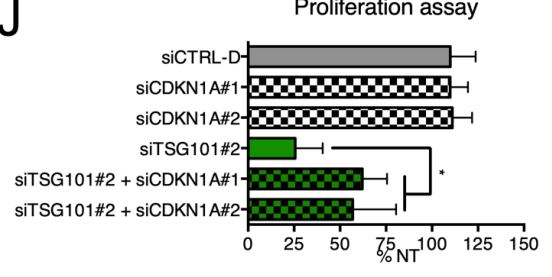

G

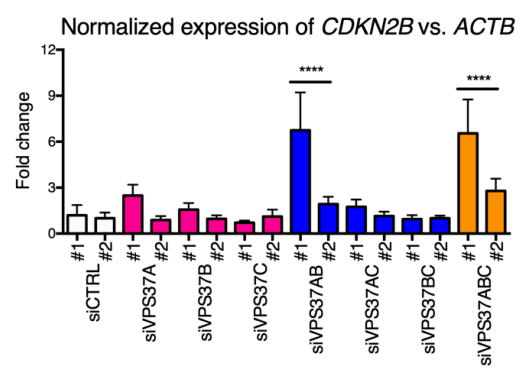

Normalized expression of

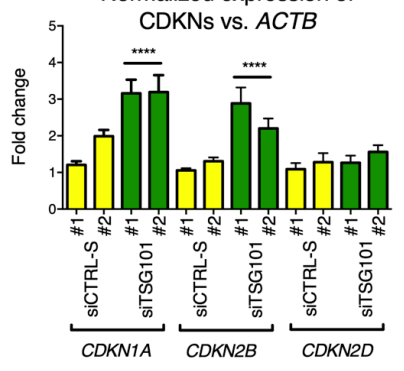

K

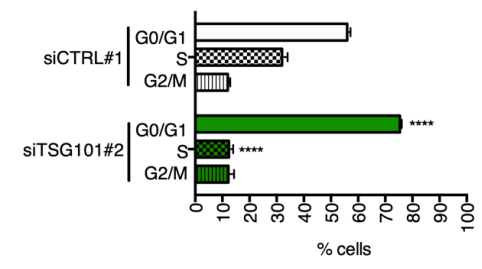


1242 Fig. S4. CRC cell growth inhibition upon knockdown of all VPS37 paralogs or TSG101. (A,

1243 B) Representative images of clone formation assessed 14 days after differential knockdown of 1244 VPS37 paralogs in (A) DLD1 and (B) RKO cells. (C) Cell proliferation of RKO cells assessed $1245120 \mathrm{~h}$ after TSG101 knockdown using the BrdU proliferation assay. (D) Clonogenic growth of 1246 RKO cells assessed 14 days after TSG101 knockdown. (E) Representative images of clone 1247 formation assessed 14 days after knockdown of TSG101 in RKO cells. (F-H) qRT-PCR analysis 1248 of expression of genes encoding CDKNs: (F) CDKN1A, (G) CDKN2B and (H) $C D K N 2 D$ in 1249 DLD1 cells. Expression of CDKNs was measured $72 \mathrm{~h}$ after individual and concurrent depletion 1250 of $\operatorname{Vps} 37$ proteins in $\mathrm{n}=5$ independent experiments. (I) qRT-PCR analysis of expression of genes 1251 encoding CDKNs: $C D K N 1 A$, $C D K N 2 B$ and $C D K N 2 D$ in RKO cells. Expression of CDKNs was 1252 measured $72 \mathrm{~h}$ after Tsg101 depletion. Non-transfected (NT) cells and transfected with non1253 targeting siRNA (siCTRL) were used to assess the basal level of the investigated genes. $A C T B$ 1254 (encoding $\beta$-Actin) was used as a reference gene. (J) Cell proliferation of RKO cells was 1255 assessed $120 \mathrm{~h}$ after concurrent silencing of TSG101 and CDKN1A using the proliferation assay. 1256 (K) Analysis of cell cycle was performed upon Tsg101 depletion. Cells were forward transfected 1257 for $96 \mathrm{~h}$, stained with PI and evaluated with a flow cytometer. Unless stated otherwise data 1258 presented in all panels are mean of $n=3$ independent experiments \pm standard deviation analyzed 1259 with one-way ANOVA with Bonferroni's correction (F-H, J) or Student's t-test (C, D, I, K). 1260 Statistical significance for grouped siCTRL conditions (A-J) and for siCTRL\#1 in panel K, * 1261 $P<0.05 ; * * P<0.01, * * * P<0.001, * * * * P<0.0001$. 


\section{Fig. $\$ 5$}
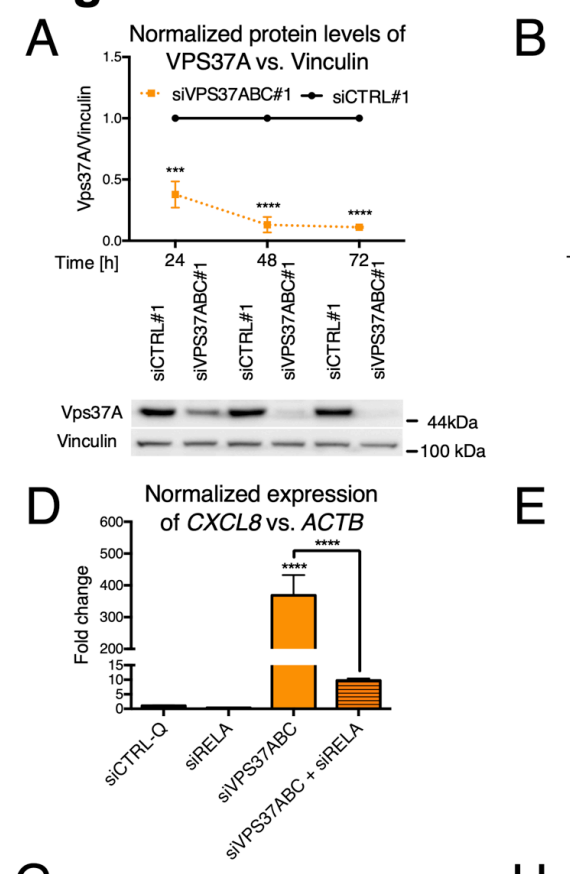

G
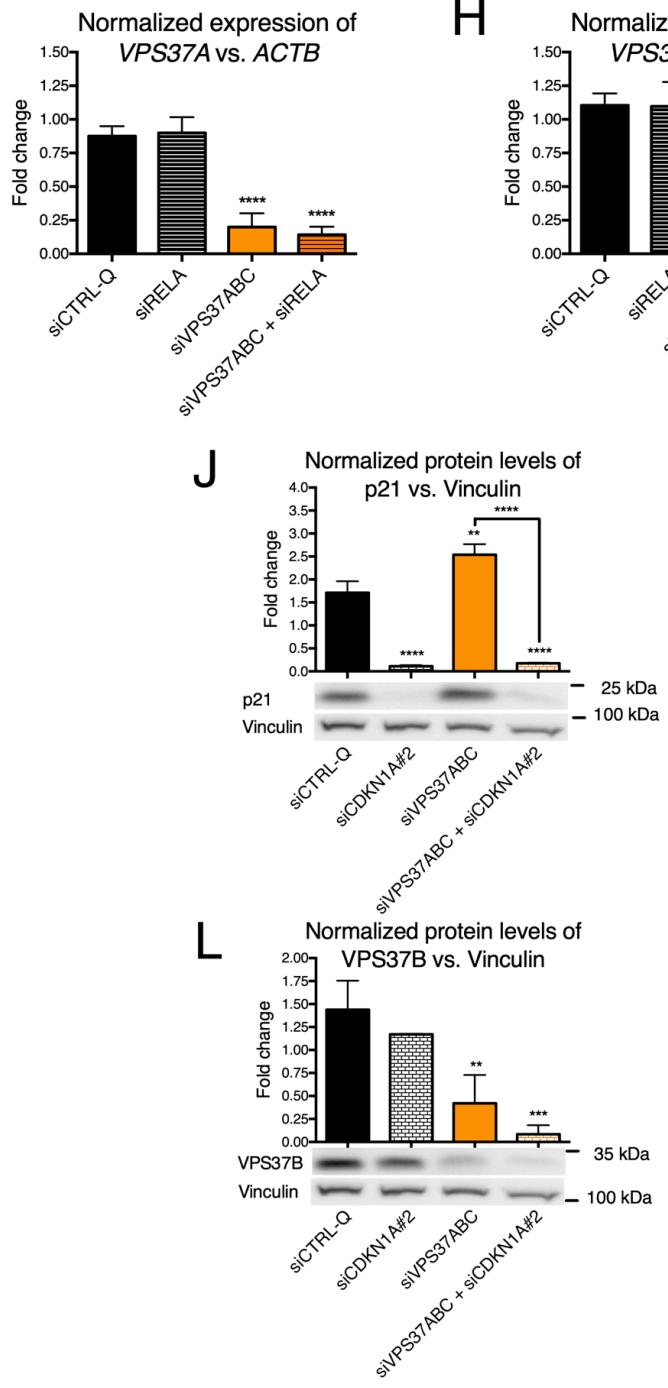

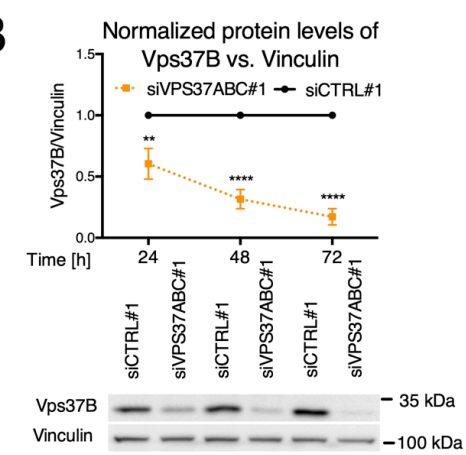

E
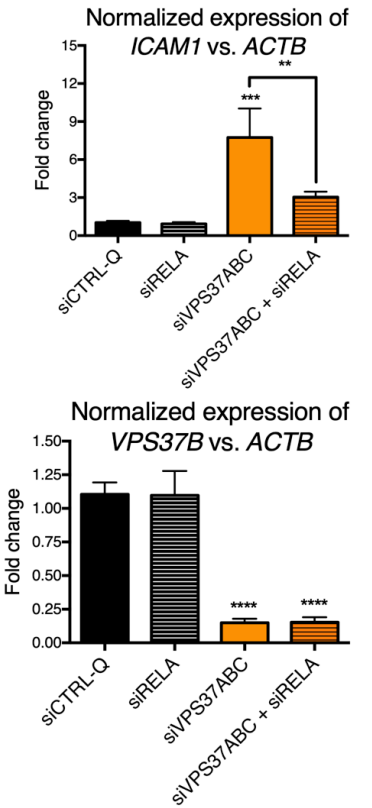

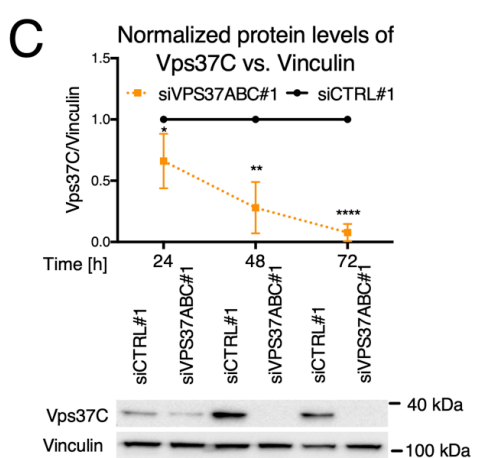

F

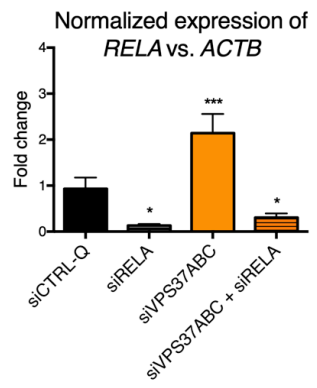

I Normalized expression of

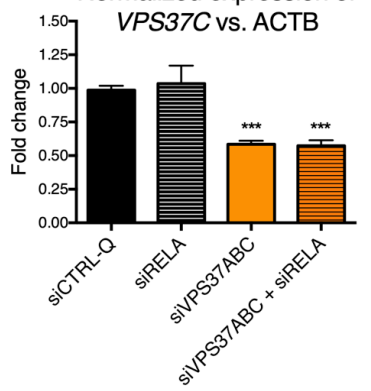

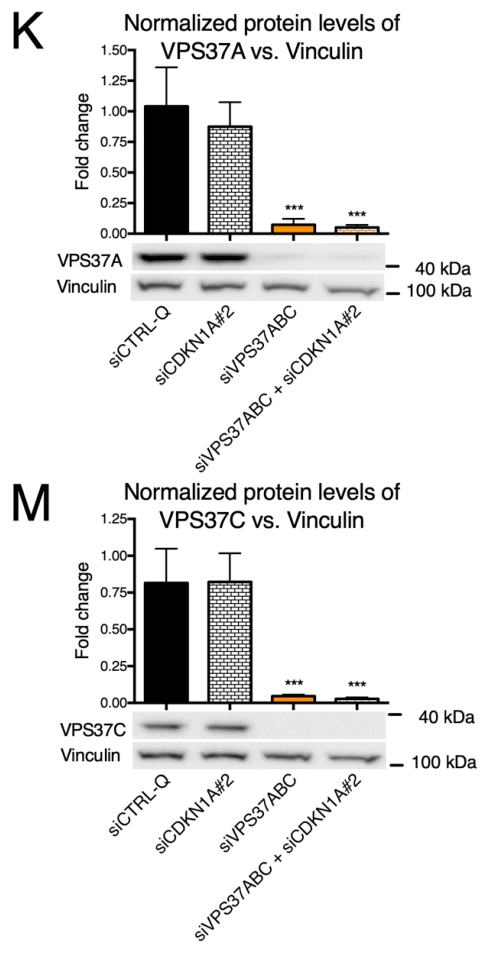


1264 Fig. S5. Control experiments corroborating independent activation of NF-кB inflammatory 1265 response and p21-mediated inhibition of cell growth. (A-C) Western blot analyses of (A) $1266 \mathrm{Vps} 37 \mathrm{~A}$, (B) Vps37B, and (C) Vps37C levels were performed 24, 48 and $72 \mathrm{~h}$ after transfection 1267 with non-targeting or on-target siRNA for $V P S 37 A B C$. (D-K) qRT-PCR analyses of (D) CXCL8, 1268 (E) ICAM1, (F) RELA, (G) VPS37A, (H) VPS37B, and (I) VPS37C and (I) were performed $72 \mathrm{~h}$ 1269 after transfection with siRNA targeting the p65 component of NF- $\kappa B$ heterodimers alone or in 1270 combination with the three VPS37 paralogs. (J-M) Western blotting analyses of (J) p21, (K) 1271 Vps37A, (L) Vps37B and (M) Vps37C abundance were performed $72 \mathrm{~h}$ after transfection with 1272 siRNA targeting $C D K N 1 A$ alone or in combination with the three VPS37 paralogs. (D-K) $A C T B$ 1273 (encoding $\beta$-Actin) was used as a reference gene in qRT-PCR analysis. (A-C, J-M) Vinculin was 1274 used as a loading control for Western blotting. Representative blots are shown along with 1275 densitometry analysis. Data in all panels are mean \pm standard deviation of $n=3$ independent 1276 experiments expressed as the fold change of either mRNA or protein abundance. In panels A-C 1277 protein abundance in siCTRL\#1 transfected cells was set to 1, whilst in panels D-I mRNA and 1278 protein abundance in non-transfected (NT) cells was set as 1. Statistical significance was 1279 assessed using unpaired Student's t-test (A-C) or one-way ANOVA test followed by 1280 Bonferroni's correction (D-M). Statistical significance against siCTRL\#1 at matching time point 1281 for transfection with three different siRNA (A-C) and siCTRL-Q for four different siRNA (D$1282 \mathrm{M}), * P<0.05, * * P<0.01, * * * P<0.001, * * * * P<0.0001$. 


\section{Fig. $\mathbf{6} 6$}
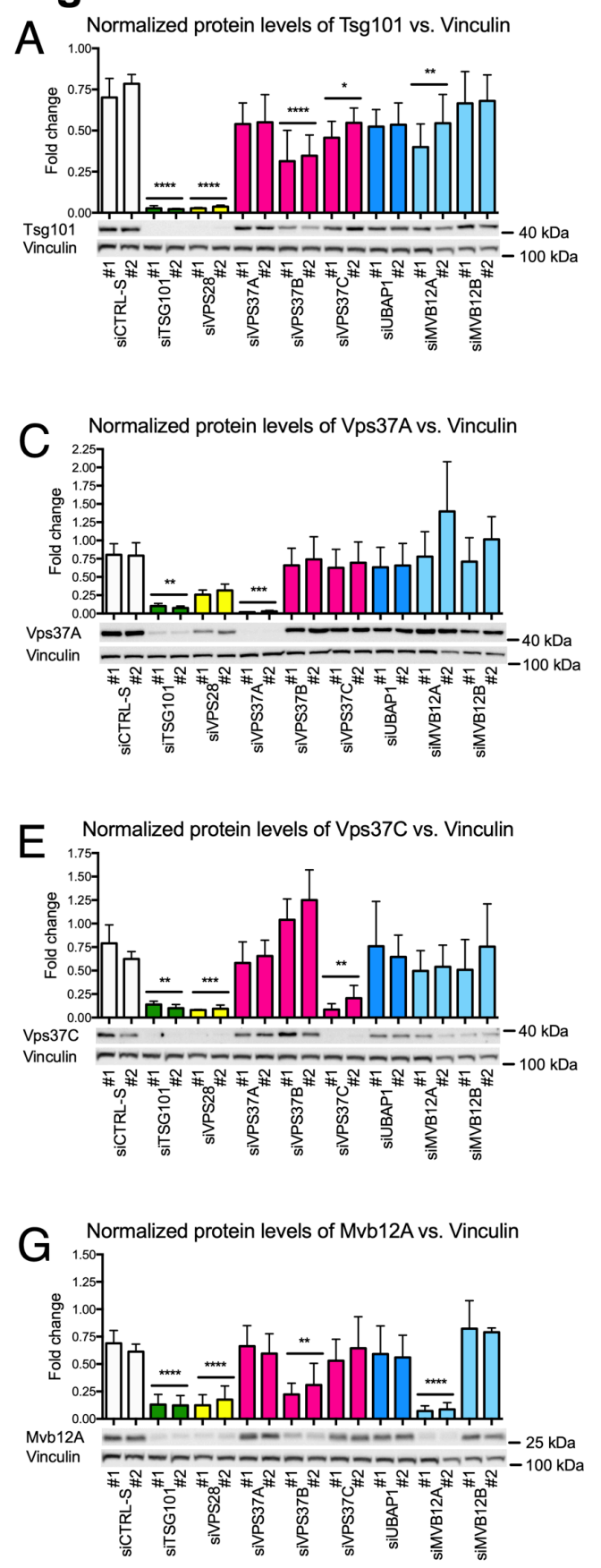
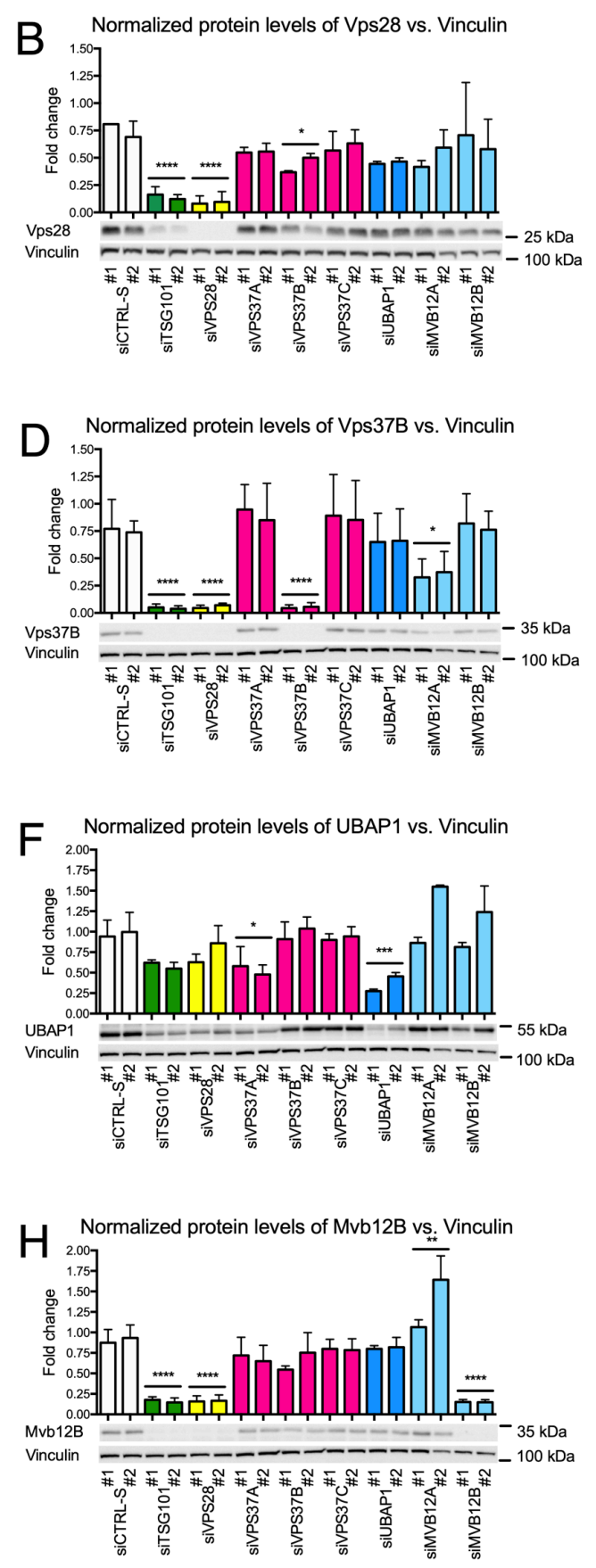

1284 Fig. S6. Destabilization of ESCRT-I subunits upon depletion of Tsg101 and Vps28. Western

1285 blotting analysis of ESCRT-I subunits: (A) Vps37A, (B) Vps37B, (C) Vps37C, (D) Tsg101, (E)

1286 Vps28, (F) UBAP-1, (G) Mvb12A and (H) Mvb12B. Lysates of RKO cells were collected $72 \mathrm{~h}$ 1287 after transfection with siRNA targeting individual ESCRT-I components. Lysates from non- 
1288 transfected cells and transfected with non-targeting siRNA were used to assess the basal level of 1289 ESCRT-I subunits. Vinculin was used as a loading control. Representative blots are shown along 1290 with densitometry analysis. Data are mean \pm standard deviation of $n=3$ independent experiments 1291 expressed as the fold change of protein level in non-transfected cells, which was set as 1. 1292 Statistical significance was assessed using ANOVA test followed by Bonferroni's correction. 1293 Statistical significance against siCTRL-S conditions, $\quad * P<0.05, \quad * * P<0.01, \quad * * * P<0.001$, $1294 \quad * * * * P<0.0001$. 
1295 Table S1. List of genes used for TCGA data mining.

1296 AAK1 ACAP2 ACTR2 ACTR3 AFTPH AKAP10 AMBRA1 AMPH ANKFY1 ANXA2 AP1B1

1297 APIG2 AP1M1 APIM2 AP1S1 AP2A1 AP2A2 AP2B1 AP2M1 AP2S1 AP3B1 AP3D1 AP3M1

1298 AP3M2 AP3S1 AP4B1 AP4E1 AP4M1 AP4S1 AP5B1 AP5M1 AP5S1 AP5Z1 APPL1 APPL2 1299 ARF1 ARF5 ARF6 ARFGAP1 ARFGAP3 ARFGEF1 ARFGEF2 ARFIP2 ARHGAP26

1300 ARHGEF18 ARRB1 ARRB2 ASAP1 ATG10 ATG12 ATG13 ATG14 ATG16L1 ATG3 ATG4A 1301 ATG4B ATG4C ATG4D ATG5 ATG7 ATG9A ATG9B ATP6V0C ATP6V1B1 ATP6V1B2 BAG4 1302 BCR BECN1 BLOC1S1 BLOC1S2 BLOC1S3 BLOC1S4 BLOC1S5 BMP2K CAND1 CAPZA1 1303 CAPZB CAV1 CAV2 CAV3 CBL CBLC CCDC112 CCDC113 CCDC121 CCDC77 CCDC93 1304 CCHCR1 CD2AP CD37 CD53 CD63 CD81 CD82 CDC42 CEP95 CHMP1A CHMP1B 1305 CHMP2A CHMP2B CHMP3 CHMP4A CHMP4B CHMP4C CHMP5 CHMP6 CHMP7 CLCN3 1306 CLCN4 CLCN5 CLCN6 CLCN7 CLTA CLTB CLTC COPA COPB1 COPB2 COPG1 COPG2 1307 CTTN DAB2 DENND5A DLL1 DNAJC6 DNM1 DNM2 DNM3 EEA1 EHD2 EPN1 EPN2 EPN3 1308 EPS15 EPS15L1 EXOC3 EXOC6 EXOC8 F8A2 FAM21A FAM21C FCHO1 FIG4 FLOT1 FLOT2 FNBP1L FSCN1 FYCO1 GABARAP GABARAPL2 GCC2 GGA1 GGA2 GGA3 GPR107 1310 GRB2 GRIPAP1 HGS HIP1 HIP1R HPS4 HSPA1A HSPA1B HSPA1L HSPA2 HSPA6 HSPA8 1311 INPP4A INPP5B INPP5D INPP5E INPP5J INPP5K ITGA1 ITGA11 ITSN1 ITSN2 KIAA0196 1312 KIAA1033 KIF16B LAMP1 LAMP2 LEPR LEPROT LGALS3 LGALS4 LIMK1 LIMK2 LYST 1313 M6PR MAPKAPK2 MCOLN1 MCOLN2 MCOLN3 MICALL1 MICALL2 MKI67 MON1A 1314 MON1B MSN MTOR MURC MVB12A MVB12B MYO5B NAPA NAPB NAPG NBEAL2 NBR1 $1315 N$ NP1 NPC2 NSF NUMB NUMBL OCRL OSBP OSBP2 OSBPL10 OSBPL11 OSBPL1A 1316 OSBPL2 OSBPL3 OSBPL5 OSBPL6 OSBPL7 OSBPL8 OSBPL9 PAK1 PAK2 PDCD6IP PI4KB 1317 PICALM PIK3C2A PIK3C2G PIK3C3 PIK3CB PIK3CD PIK3CG PIK3R4 PIKFYVE PIP4K2B 1318 PIP4K2C PIP5K1C PIP5KL1 PLEKHM1 PLIN3 PMEL POM121 PRKCDBP PSMA7 PTEN 1319 PTPMT1 PTRF RAB11A RAB11B RAB11FIP1 RAB11FIP2 RAB11FIP3 RAB11FIP4 1320 RAB11FIP5 RAB12 RAB13 RAB14 RAB15 RAB17 RAB1B RAB20 RAB21 RAB22A RAB24 $1321 \quad R A B 25 \quad R A B 26 \quad R A B 27 A \quad R A B 27 B \quad R A B 29 \quad R A B 31 \quad R A B 35 \quad R A B 37 \quad R A B 38$ RAB3B $R A B 3 D$ 1322 RAB3IL1 RAB3IP RAB4A RAB4B RAB5A RAB5B RAB5C RAB7A RAB7B RAB9A RAB9B 1323 RABEP1 RABEP2 RABEPK RABGEF1 RABIF RAC1 RALBP1 RBSN REP15 REPS1 REPS2 1324 RHOA RHOBTB3 RICTOR RILP RIMS1 RNF115 ROCK1 ROCK2 RPTOR RUBCN RUFY1 
1325 SACM1L SCAMP1 SCAMP2 SCAMP3 SCAMP4 SCAMP5 SCARB2 SCYL2 SDCBP SDPR 1326 SGSM2 SH3GL1 SH3GL2 SH3GL3 SH3GLB1 SH3GLB2 SH3TC2 SMAP1 SNAP23 SNAP91

1327 SNF8 SNX1 SNX10 SNX11 SNX12 SNX13 SNX14 SNX15 SNX16 SNX17 SNX18 SNX19 SNX2 1328 SNX20 SNX21 SNX22 SNX24 SNX25 SNX27 SNX4 SNX5 SNX6 SNX7 SNX8 SNX9 SOS1 SPC25 1329 SPIRE1 SQSTM1 STAM STAM2 STAMBP STX11 STX12 STX6 STX7 SURF4 SYNJ1 SYNJ2 1330 SYNRG SYTL4 TBC1D10A TBC1D10B TBC1D10C TBC1D14 TBC1D2B TBC1D5 TMEM55A 1331 TNIK TNK2 TPCN1 TPCN2 TPTE2 TRIM4 TSG101 TYRP1 UBAP1 UBQLN2 ULK1 ULK3 1332 UNC119 UNC13A UNC13B UNC13C UNC13D USP8 VAC14 VAMP3 VAMP4 VAMP7 VAMP8

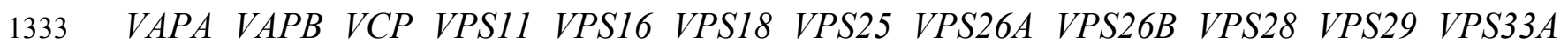
1334 VPS33B VPS35 VPS36 VPS37A VPS37B VPS37C VPS37D VPS39 VPS41 VPS45 VPS4A VPS4B 1335 VPS52 VPS53 VPS54 VTA1 VTI1B WAS WASH1 WASL WDFY3 WDR44 WIPI1 ZFYVE16 1336 ZFYVE27 ZFYVE9. 
1337 Table S2. List of differentially expressed genes in the early stages of CRC.

1338 ARHGEF18, BAG4, CD82, FAM21C, HPS4, ITSN1, LAMP2, MICALL1, PIK3CD, PRKCDBP,

1339 RAB12, RAB3IL1, RABEPK, SCAMP5, SMAP1, SNX1, SNX8, SNX9, SPIRE1, WASL. 
1340 Table S3. List of differentially expressed genes in the advanced stages of CRC.

1341 ANXA2, ARFGEF2, BLOC1S3, CD37, DNM1, DNM3, GABARAP, HIP1, INPP5J, LAMP1,

1342 NPC2, OSBPL8, SNX11, SNX25, STAM, STX11, STX6, SYTL4, VCP, VPS37B, WAS. 
1343 Table S4. Expression of genes encoding ESCRT-I subunits in COAD and READ cohorts of 1344 TCGA patients at the early (stage I and II) and advanced stages (stage III and IV) of CRC.

1345 Table shows fold change ratio between the matched healthy and cancer patient tissue in the 1346 logarithmic scale (Log2FC); with associated false discovery rate (FDR) upon differential 1347 expression analysis using TCGAbiolinks.

1348

\begin{tabular}{|l|c|c|c|c|}
\hline \multicolumn{1}{|c}{ Gene } & \multicolumn{2}{c}{ Early stages } & \multicolumn{2}{c|}{ Advanced stages } \\
& Log2FC & FDR & -0.308 & FDR \\
\hline VPS37A & -0.294 & 0.09 & -0.686 & 0.23 \\
\hline VPS37B & -0.266 & 0.15 & -0.102 & 0.02 \\
\hline VPS37C & -0.070 & 0.64 & -0.136 & 0.71 \\
\hline TSG101 & -0.006 & 0.97 & 0.198 & 0.66 \\
\hline VPS28 & -0.439 & 0.01 & 0.52 \\
\hline
\end{tabular}

1349 
1352 status, $\mathrm{G}$ - disease grade.

1353

\begin{tabular}{|l|l|l|l|}
\hline \multicolumn{2}{|c}{$\begin{array}{c}\text { Vps37A staining } \\
\text { intensity }\end{array}$} & \multicolumn{2}{c|}{$\begin{array}{c}\text { Vps7B staining } \\
\text { intensity }\end{array}$} \\
\hline $\mathbf{1}$ & 2 & 2 & 2 \\
\hline $\mathbf{2}$ & 2 & 1 & 4 \\
\hline $\mathbf{2}$ & 2 & 2 & 13 \\
\hline $\mathbf{3}$ & 2 & 1 & 22 \\
\hline $\mathbf{3}$ & 2 & 2 & 43 \\
\hline $\mathbf{3 a}$ & 2 & 2 & 1 \\
\hline $\mathbf{3 b}$ & 2 & 1 & 1 \\
\hline $\mathbf{3 b}$ & 2 & 2 & 1 \\
\hline $\mathbf{3 c}$ & 2 & 1 & 1 \\
\hline $\mathbf{3 c}$ & 2 & 2 & 1 \\
\hline $\mathbf{4 a}$ & 2 & 1 & 1 \\
\hline $\mathbf{4 a}$ & 2 & 2 & 4 \\
\hline $\mathbf{4 b}$ & 2 & 1 & 1 \\
\hline $\mathbf{4 b}$ & 2 & 2 & 5 \\
\hline
\end{tabular}

1354

\begin{tabular}{|l|l|l|l|}
\hline \multicolumn{2}{|c}{ pN status $\begin{array}{c}\text { Vps37A staining } \\
\text { intensity }\end{array}$} & \multicolumn{2}{c|}{$\begin{array}{c}\text { Vps37B staining } \\
\text { intensity }\end{array}$} \\
\hline 0 & 2 & 1 & 15 \\
\hline $\mathbf{0}$ & 2 & 2 & 48 \\
\hline $\mathbf{1 a}$ & 2 & 1 & 5 \\
\hline $\mathbf{1 a}$ & 2 & 2 & 12 \\
\hline $\mathbf{1 b}$ & 2 & 2 & 7 \\
\hline 1c & 2 & 1 & 2 \\
\hline $\mathbf{1 c}$ & 2 & 2 & 1 \\
\hline $\mathbf{2 a}$ & 2 & 1 & 5 \\
\hline $\mathbf{2 a}$ & 2 & 2 & 2 \\
\hline $\mathbf{2 b}$ & 2 & 1 & 3 \\
\hline
\end{tabular}

1355

\begin{tabular}{|l|l|l|l|}
\hline \multicolumn{2}{|c}{ G $\begin{array}{c}\text { Vps37A staining } \\
\text { intensity }\end{array}$} & \multicolumn{2}{c|}{$\begin{array}{c}\text { Vps37B staining } \\
\text { intensity }\end{array}$} \\
\hline $\mathbf{2}$ & 2 & 1 & 21 \\
\hline $\mathbf{2}$ & 2 & 2 & 51 \\
\hline $\mathbf{3}$ & 2 & 1 & 9 \\
\hline $\mathbf{3}$ & 2 & 2 & 19 \\
\hline
\end{tabular}


1357 Table S6. List of differentially expressed genes in COAD and READ cohorts of TCGA 1358 patients at the early (stage I and II) and advanced stages (stage III and IV) of CRC. Table is 1359 available as a supplemental file for this manuscript. Table shows fold change ratio between the

1360 matched healthy and cancer patient tissue in the logarithmic scale (Log2FC); with associated 1361 false discovery rate (FDR) upon differential expression analysis using TCGAbiolinks. Genes 1362 with increased and decreased expression are those with $\mathrm{FDR}<0.05$ and $\log 2 \mathrm{FC} \geq 0.6$ and $\leq-0.6$, 1363 respectively. Empty records signify no significant changes for the given gene. 
Table S7. List of siRNA combinations used in the present study.

\begin{tabular}{|c|c|c|c|c|}
\hline Silencing condition & SiRNA\#1 & siRNA\#2 & siRNA\#3 & SiRNA\#4 \\
\hline \multicolumn{5}{|c|}{ Single silencing } \\
\hline siCTRL-S\#1 & 4390843 & & & \\
\hline siCTRL-S\#2 & 4390846 & & & \\
\hline SiTSG101\#1 & s14439 & & & \\
\hline SiTSG101\#2 & s14440 & & & \\
\hline SiVPS28\#1 & s27577 & & & \\
\hline SiVPS28\#2 & s27579 & & & \\
\hline siVPS37A\#1 & s44037 & & & \\
\hline siVPS37A\#2 & s44038 & & & \\
\hline siVPS37B\#1 & s36177 & & & \\
\hline siVPS37B\#2 & s36178 & & & \\
\hline siVPS37C\#1 & s30059 & & & \\
\hline siVPS37C\#2 & $\mathrm{s} 30060$ & & & \\
\hline siUBAP1\#1 & s27812 & & & \\
\hline siUBAP1\#1 & s27813 & & & \\
\hline SiMVB12A\#1 & s41121 & & & \\
\hline SiMVB12A\#2 & s41122 & & & \\
\hline SiMVB12B\#1 & s40157 & & & \\
\hline SiMVB12B\#2 & $\mathrm{s} 40158$ & & & \\
\hline \multicolumn{5}{|c|}{ Double silencing } \\
\hline siCTRL-D\#1 & 4390843 & 4390843 & & \\
\hline siTSG101-D\#2 & s14440 & 4390843 & & \\
\hline siTSG101\#2+CDKN1A\#1 & s14440 & s415 & & \\
\hline siTSG101\#2+CDKN1A\#2 & s14440 & s417 & & \\
\hline \multicolumn{5}{|c|}{ Triple silencing } \\
\hline siCTRL\#1 & 4390843 & NC3 & NC4 & \\
\hline SiCTRL\#2 & 4390843 & 4390846 & NC4 & \\
\hline siVPS37A\#1 & s44037 & 4390843 & NC4 & \\
\hline SiVPS37A\#2 & s44038 & 4390843 & NC4 & \\
\hline siVPS37B\#1 & s36177 & 4390843 & NC4 & \\
\hline siVPS37B\#2 & s36178 & 4390843 & NC4 & \\
\hline siVPS37C\#1 & s30059 & 4390843 & $\mathrm{NC4}$ & \\
\hline siVPS37C\#2 & s30060 & 4390843 & NC4 & \\
\hline SiVPS37AB\#1 & s44037 & s36177 & 4390843 & \\
\hline SiVPS37AB\#2 & s44038 & s36178 & 4390843 & \\
\hline SiVPS37AC\#1 & s44037 & s30059 & 4390843 & \\
\hline SiVPS37AC\#2 & s44038 & s30060 & 4390843 & \\
\hline SiVPS37BC\#1 & s36177 & s30059 & 4390843 & \\
\hline SiVPS37BC\#2 & s36178 & s30060 & 4390843 & \\
\hline siVPS37ABC\#1 & s44037 & s36177 & s30059 & \\
\hline siVPS37ABC\#2 & s44038 & s36178 & s30060 & \\
\hline siTSG101\#2 & s14440 & 4390846 & $\mathrm{NC4}$ & \\
\hline \multicolumn{5}{|c|}{ Quadruple silencing } \\
\hline siCTRL-Q & 4390843 & 4390846 & NC3 & $\mathrm{NC} 4$ \\
\hline siVPS37ABC & s44037 & s36177 & s30059 & 4390843 \\
\hline siCDKN1A\#1 & $\mathrm{s} 415$ & 4390843 & NC3 & $\mathrm{NC} 4$ \\
\hline siCDKN1A\#2 & s417 & 4390843 & NC3 & $\mathrm{NC4}$ \\
\hline
\end{tabular}


bioRxiv preprint doi: https://doi.org/10.1101/2020.07.02.183954; this version posted July 3, 2020. The copyright holder for this preprint (which was not certified by peer review) is the author/funder. All rights reserved. No reuse allowed without permission.

\begin{tabular}{|l|l|l|l|l|}
\hline siRELA & $\mathrm{s} 11916$ & 4390843 & $\mathrm{NC3}$ & $\mathrm{NC4}$ \\
\hline siVPS37ABC+siCDKN1A\#1 & $\mathrm{s} 44037$ & $\mathrm{~s} 36177$ & $\mathrm{~s} 30059$ & $\mathrm{~s} 415$ \\
\hline siVPS37ABC+siCDKN1A\#2 & $\mathrm{s} 44037$ & $\mathrm{~s} 36177$ & $\mathrm{~s} 30059$ & $\mathrm{~s} 417$ \\
\hline siVPS37ABC+siRELA & $\mathrm{s} 44037$ & $\mathrm{~s} 36177$ & $\mathrm{~s} 30059$ & $\mathrm{~s} 11916$ \\
\hline
\end{tabular}


Table S8. List of antibodies used in the present study. Abbreviations: WB - Western blot, IHC

$1368-$ Immunohistochemistry.

1369

\begin{tabular}{|c|c|c|c|c|c|}
\hline Antibody & Species & Technique & Dilution & Company & $\begin{array}{l}\text { Catalog } \\
\text { number }\end{array}$ \\
\hline VPS37A & Rabbit & WB, IHC & $\begin{array}{l}\text { WB 1:1000 } \\
\text { IHC 1:100 }\end{array}$ & Proteintech & 11870-1-AP \\
\hline VPS37B & Rabbit & $\mathrm{WB}, \mathrm{IHC}$ & $\begin{array}{l}\text { WB 1:1000 } \\
\text { IHC 1:100 }\end{array}$ & Sigma & HPA-038218 \\
\hline VPS37C & Goat & WB & WB 1:500 & Abcam & $\mathrm{Ab} 40851$ \\
\hline TSG101 & Rabbit & WB & WB 1:1000 & Abcam & Ab133586 \\
\hline VPS28 & Rabbit & WB & WB 1:1000 & Abcam & Ab167172 \\
\hline UBAP1 & Rabbit & WB & WB 1:1000 & Proteintech & 12385-1-AP \\
\hline MVB12A & Rabbit & WB & WB 1:1000 & Atlas Antibodies & HPA041885 \\
\hline MVB12B & Rabbit & WB & WB 1:1000 & Atlas Antibodies & HPA043683 \\
\hline Phospho-p65 & Rabbit & WB & WB 1:1000 & $\begin{array}{l}\text { Cell Signaling } \\
\text { Technology }\end{array}$ & 3033 \\
\hline p65 & Mouse & WB & WB 1:1000 & $\begin{array}{l}\text { Cell Signaling } \\
\text { Technology }\end{array}$ & 6956 \\
\hline p100/p52 & Rabbit & WB & WB 1:1000 & $\begin{array}{l}\text { Cell Signaling } \\
\text { Technology }\end{array}$ & 4882 \\
\hline Phospho-JNK & Rabbit & WB & WB 1:500 & $\begin{array}{l}\text { Cell Signaling } \\
\text { Technology }\end{array}$ & 9255 \\
\hline Phospho-ERK & Rabbit & WB & WB 1:1000 & $\begin{array}{l}\text { Cell Signaling } \\
\text { Technology }\end{array}$ & 9101 \\
\hline Phospho-p38 & Rabbit & WB & WB 1:500 & $\begin{array}{l}\text { Cell Signaling } \\
\text { Technology }\end{array}$ & 9216 \\
\hline p21 & Rabbit & WB & WB 1:2000 & $\begin{array}{l}\text { Cell Signaling } \\
\text { Technology }\end{array}$ & 2947 \\
\hline Vinculin & Mouse & WB & WB 1:5000 & Sigma-Aldrich & V9131 \\
\hline
\end{tabular}


Table S9. List of primers used in the present study.

1372

\begin{tabular}{|l|l|l|}
\hline $\begin{array}{l}\text { Gene of } \\
\text { interest }\end{array}$ & Forward primer sequence & Reverse primer sequence \\
\hline$A C T B$ & CAGGTCATCACCATTGGCAAT & TCTTTGCGGATGTCCACGT \\
\hline$G A P D H$ & CATGTTCGTCATGGGTGTGA & GTGATGGCATGGACTGTGGT \\
\hline$C D K N 1 A$ & TGCCGAAGTCAGTTCCTTGT & GTTCTGACATGGCGCCTCC \\
\hline$C D K N 2 B$ & GGGACTAGTGGAGAAGGTG & CATCATCATGACCTGGATCGC \\
\hline$C D K N 2 D$ & CGCTGCAGGTCATGATGTTT & GGGTGTCCAGGAATCCAGTG \\
\hline$C X C L 8$ & GCTCTCTTGGCAGCCTTCCTGA & TTTCCTTGGGGTCCAGACAGAGC \\
\hline$I C A M 1$ & GGAGCCCGCTGAGGTCACGA & AGTCGCTGGCAGGACAAAGGT \\
\hline$N F K B I A$ & CGCCCAAGCACCCGGATACA & AGGGCAGCTCGTCCTCTGTGA \\
\hline$R E L A$ & AGCTTGTAGGAAAGGACTGCC & ATAGGAACTTGGAAGGGGTTGTTT \\
\hline$T N F A I P 3$ & GTGATCGGCCCCCAGAGGGA & TGAGGGTTTGCTACAACATGGGC \\
\hline$V P S 37 A$ & TAGTGAGAGCTGTAGTGCAAGTGCC & TCGCCTGCTGAAGTTTCTCTTCCT \\
\hline$V P S 37 B$ & ACGTTGAAAGCACGCTTGAC & TGCTAACAGGGTCTCCAAGG \\
\hline$V P S 37 C$ & GGAAGGCATGAAGATCGAAG & TTCCTCACCACTTCCTGGAG \\
\hline
\end{tabular}

1373 